\author{
A Thesis \\ presented to \\ the Faculty of California Polytechnic State University,
}

San Luis Obispo

In Partial Fulfillment

of the Requirements for the Degree

Master of Science in Aerospace Engineering

by

Sean Crowley

June 2019 
(C) 2019

Sean Crowley

ALL RIGHTS RESERVED 
COMMITTEE MEMBERSHIP

TITLE: A CUBESAT ORBITAL TEST PLATFORM FOR AN ELECTROTHERMAL PLASMA MICRO-THRUSTER

AUTHOR: Sean Crowley

DATE SUBMITTED: June 2019

COMmitTeE CHAIR: Amelia Greig, Ph.D.

Assistant Professor of Aerospace Engineering

COMMITTEE MEMBER: Jesse Mills, M.S.

Member of Technical Staff, MIT Lincoln Laboratory

COMMitTeE MEMBER: Kira Abercromby, Ph.D.

Associate Professor of Aerospace Engineering

COMmitTeE MemBeR: Pauline Faure, Ph.D.

Assistant Professor of Aerospace Engineering 


\title{
ABSTRACT \\ A CUBESAT ORBITAL TEST PLATFORM FOR AN ELECTROTHERMAL PLASMA MICRO-THRUSTER
}

\author{
Sean Crowley
}

As the viability and capability of CubeSats increases, a demand for propulsion systems is becoming apparent. The limitations put forth in the "CubeSat Design Specifications" [1] and the additional difficulties of low available Size, Weight and Power (SWaP) for propulsion systems aboard CubeSats have left a technological gap that many are trying to fill. One class of propulsion, micro-propulsion systems, fills this gap nicely as they generally have low size and weight with a reasonable amount of power draw. Many can function for reasonable lifetime scales utilizing pressure vessels under one atmosphere and less then 100 watt-hours of stored chemical energy as stipulated by the CubeSat standards. Micro-thruster systems can provide station keeping to extend mission life, a method to de-orbit, or possibly even bulk position change capabilities to CubeSats, thus increasing their ever growing usage potential. Many promising micro-propulsion systems have low Technology Readiness Level (TRL) and have limited-to-no space flight heritage, making their selection for a CubeSat mission more risky. A rapid, low cost, development and demonstration platform for advanced micro-propulsion systems is needed to increase TRL of promising technologies and provide them with space flight heritage, thus making them low risk options for future mission planners.

CubeSats themselves represent an interesting test bed platform for emerging propulsion technologies as they are relatively inexpensive and less risk adverse than traditional space systems. This endeavor strives to analyze the efficacy of using CubeSats as a micro-propulsion demonstration platform to be built by students of the Califor- 
nia Polytechnic State University's PolySat Team. This thesis will baseline the use of Pocket Rocket, an electrothermal plasma micro-thruster, originally developed by the Australian National University. The thruster has seen continued development with the PolySat team, and the PolySat version's performance and form factor is used in the following analyses. However, the platform proposed herein would not be inherently limited to this specific micro-propulsion technology.

The following analyses shows that a sun pointing control law utilizing additional deployable panels is necessary for the power budget to close. The necessary area is equal to a $2 \times 3 \mathrm{U}$ surface of panels, which will be comprised of one $1 \times 3 \mathrm{U}$ face and two $1 / 2 \times 3 \mathrm{U}$ deployable panels. It is also apparent that the thermal environment requires intervention to be survivable. More details will be provided in the design of an extremely SWaP efficient passive thermal management system. It also highlights the viability of the of utilizing two thrusters to impose a tumble that is easily measured. It highlights the additional complexity involved by an increasingly unbalanced craft, and the need to know the inertia tensor of the craft to accurately predict the flight functionality of the thruster. Two 1 milliNewton thrusters firing for a period of 20 seconds results in a tumble across the intended axis of about $.18 \mathrm{hZ}$. This is easily measurable and also recoverable based on the selected reaction wheel bundle. 


\section{ACKNOWLEDGMENTS}

I would like to extend a sincere thank you to my thesis committee who promptly provided advice and direction to aid me in completing this thesis. I would also like to thank my friends and family, without whom, I would not have been able to complete this undertaking. I know I spent the majority of my last year talking about this with you, so thank you for listening to me and providing input and advice. Mom and Dad, thank you for believing in me. Conor and Peter, thanks for pretending nursing is more difficult than rocket science. I am immensely proud of this accomplishment, which differs drastically from the path I envisioned for my life. A special thanks first to Kurt Giessler, who showed a high school kid what engineering really is. Secondly,

to Jesse Mills, who showed that same kid he was capable of more than just getting his hands dirty. I owe this to all of you more than you realize, and thank you from the bottom of my heart for getting me here. Enjoy! 


\section{TABLE OF CONTENTS}

Page

LIST OF TABLES ....................... . . . . ix

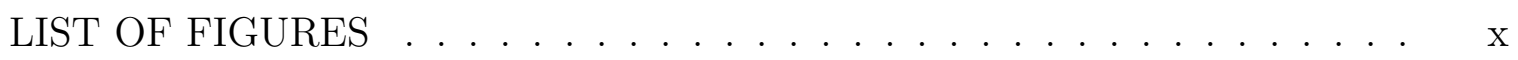
CHAPTER

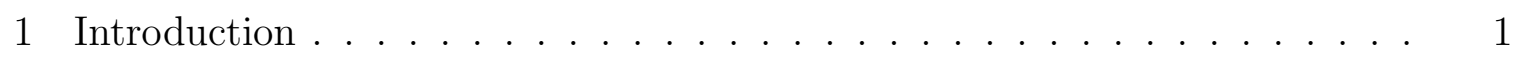

1.1 Problem Statement . . . . . . . . . . . . . . . . . . . . . 1

1.2 Proposed Solution . . . . . . . . . . . . . . . . . . . 2

1.3 Previous Research . . . . . . . . . . . . . . . . . . . . . 2

1.4 Expected Scope . . . . . . . . . . . . . . . . . 6

2 Hardware Considerations . . . . . . . . . . . . . . . . . . 7

2.1 Critical Components and Subsystems . . . . . . . . . . 7

2.2 Selection Details $\ldots \ldots \ldots \ldots \ldots$

2.3 Pocket Rocket Review . . . . . . . . . . . . . . . . . . . . 12

2.4 Proposed CONOPS . . . . . . . . . . . . . . . . . . . . . 14

3 Analysis . . . . . . . . . . . . . . . . . . 17

3.1 Introduction and Orbit Selection $\ldots \ldots \ldots \ldots \ldots$

3.2 Power Analysis . . . . . . . . . . . . . . . . . . . . . . 19

3.3 Thermal Model . . . . . . . . . . . . . . . . . . . . . 27

3.4 Communications Budget . . . . . . . . . . . . . . . . . . 39

3.5 Dynamics Model . . . . . . . . . . . . . . . . . . 44

4 Future Work . . . . . . . . . . . . . . . . . . . 59

4.1 Identifying Potential Future Student Work . . . . . . . . . . . 59

4.2 Future Mission $\ldots \ldots \ldots \ldots \ldots \ldots \ldots \ldots \ldots \ldots \ldots \ldots$ 


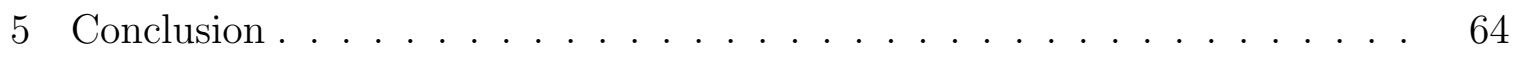

BIBLIOGRAPHY ............................. 67 


\section{LIST OF TABLES}

Table

Page

2.1 Table of Hardware Selections _. . . . . . . . . . . 10

3.1 Table of Power Consumption Rates . . . . . . . . . . . . . . 21

3.2 Table of Internal Heat Generation . . . . . . . . . . . . . . 31

3.3 Table of Uplink and Downlink Margin Compared to Antenna Gain 42 


\section{LIST OF FIGURES}

Figure

Page

1.1 Generated Image of Proposed CubeSat in Orbit . . . . . . . . . . . 3

2.1 Exploded View of CubeSat Design with Component Labels . . . . . 11

2.2 Cross Section of the CPCL Pocket Rocket . . . . . . . . . . . 13

2.3 Diagram of Coupled Torques Imposing Tumble on Representative CubeSat . . . . . . . . . . . . . . . . 15

2.4 Notional Timeline of CubeSat Operations . . . . . . . . . . 16

3.1 Figure Displaying Proposed Orbit of CubeSat . . . . . . . . . . 18

3.2 Power Generation on All Six Faces with Nadir Pointing . . . . . . . 22

3.3 Power Generation on All Six Faces with Nadir Pointing on Day 300 in Previous Plot. . . . . . . . . . . . . . . . 23

3.4 Image to Label Sides and Position of Thrusters for Reference . . . . 24

3.5 Power Generation on Single Face with Sun Pointing . . . . . . . . . 25

3.6 Power Generation on Single Face with Sun Pointing on Day 300 of Previous Plot ................... 25

3.7 Battery Level Using a Single Face with Sun Pointing . . . . . . . 26

3.8 Power Generation on Single Face and Deployables with Sun Pointing 27

3.9 Power Generation on Single Face and Deployables with Sun Pointing on Day 300 in Previous Plot . . . . . . . . . . . . . . . 27

3.10 Battery Charge for Single Face and Deployables with Sun Pointing 28

3.11 Diagram Detailing the Thermal Environment as Resistances . . . . 29

3.12 Spacecraft Temperatures, Bare Aluminum Shell in Full Sun . . . . 33

3.13 Spacecraft Temperatures, Bare Aluminum shell, Eclipsing . . . . . 34 
3.14 Spacecraft Temperatures, Black Paint in Full Sun . . . . . . . . . . 35

3.15 Spacecraft Temperatures, Black Paint, Eclipsing . . . . . . . . . 35

3.16 Spacecraft Temperatures, Gray Paint, Full Sun . . . . . . . . . 36

3.17 Spacecraft Temperatures, Gray Paint, Eclipsing . . . . . . . . . . 37

3.18 Spacecraft Temperatures, Gray Paint \& Thermal Straps, Full Sun . 38

3.19 Spacecraft Temperatures, Gray Paint \& Thermal Straps, Eclipsing . 38

3.20 Bit Error Probability as a Function of $E_{b} / N_{O} \ldots \ldots$. . . . . . 41

3.21 Spacecraft Groundtrack, Communication Window Represented by Star Square . . . . . . . . . . . . . . . . . . 43

3.22 Components of the Thrust Force and Various Lever Arms Within the Craft and Their Relation to the End Rotational Rates of the craft 48

3.23 Resultant Tumble When Thruster Is Perfectly Aligned and Inertia Tensor is Balanced . . . . . . . . . . . . . . . . . 49

3.24 Resultant Tumble on Unintended Axis as the Thrust Angle Changes and Inertia Tensor is Balanced . . . . . . . . . . . . . . . . . 49

3.25 Resultant Tumble on Intended Axis as the Thrust Angle Changes and Inertia Tensor is Balanced . . . . . . . . . . . . 50

3.26 Resultant Tumble When Thruster Is Perfectly Aligned and Inertia Tensor is Unbalanced . . . . . . . . . . . . . . . . . . 51

3.27 Resultant Tumble on Unintended Axis as the Thrust Angle Changes and Inertia Tensor is Unbalanced . . . . . . . . . . . . . . . . 51

3.28 Resultant Tumble on Intended Axis as the Thrust Angle Changes and Inertia Tensor is Unbalanced . . . . . . . . . . . . . . . 52

3.29 Resultant Tumble When Thruster Is Perfectly Aligned and Worst Case Inertia Tensor . . . . . . . . . . . . . . . . . . . . . . . . . 52

3.30 Resultant Tumble on Unintended Axis as the Thrust Angle Changes and Worst Case Inertia Tensor . . . . . . . . . . . . . . . 53

3.31 Resultant Tumble on Intended Axis as the Thrust Angle Changes and Worst Case Inertia Tensor . . . . . . . . . . . . . . 53 
3.32 No Misalignment of Thruster Compared Between Different Inertia Tensors . . . . . . . . . . . . . . . . . . . . 54

3.33 Thruster Repositioned to Maximize Roll, With a Balanced Inertia Tensor . . . . . . . . . . . . . . . . . . . . . 55

3.34 Plot of Disturbance Torques Effects as Incident Angle Varies . . . . 57 
Chapter 1

INTRODUCTION

\subsection{Problem Statement}

Micro-propulsion technology has seen major advancement over the previous decades making these systems more viable for a variety of missions. Improved Size, Weight, and Power (SWaP) has resulted in micro propulsion systems that could be implemented in a variety of spacecraft ranging from CubeSats to Geosynchronous spacecraft. One of the last major hurdles to overcome in the use of these systems is the difficulty in reaching a high enough Technology Readiness Level (TRL) to be effectively marketable for operational use. Historically, to reach a technology readiness level of 7 and establish a flight heritage, a propulsion system would be selected for a larger mission as a secondary scientific payload. As a condition of their use, the systems would typically see extensive testing and certification. The systems selected are typically from major manufacturers of aerospace technology. Both for their marketability and access to funds and infrastructure necessary to test their system to acceptable levels demanded by the management of the flight mission. This effectively limits the flight of new propulsion technologies to these larger companies. However, the development of new technology is most certainly not limited to these same companies. A new method to reach a TRL of 7 needs to be developed to allow the testing of new propulsion technologies within the means of any technology developer, whether it be a school, start-up, or major aerospace retailer. 


\subsection{Proposed Solution}

CubeSats have become a viable alternative for a variety of missions; they are more cost efficient and less risk adverse. This makes them a strong candidate for orbital test bed platforms. CubeSats are also simpler than alternative satellite systems, namely due to the "plug and play" methodology utilized by their common use component design. By designing a CubeSat that can test micro-propulsion in orbit, on a student team budget, the ability to test a micro-propulsion system is open to more technology developers. This allows more systems to increase the technology readiness level and establish a flight heritage. This is an important step as it allows smaller companies to test and get accurate performance metrics for their micro-propulsion system. This in turn makes selection for future missions more robust and will allow selection of the best system from a larger applicant pool. Figure 1.1 depicts the proposed CubeSat in orbit about Earth.

\subsection{Previous Research}

The initial CubeSat concept was recommended by Jordi Puig-Suari and Bob Twiggs in 2000, and was published in "Development of the Standard CubeSat Deployer and a CubeSat Class PicoSatellite" in 2001 [2]. This initial paper, along with the "CubeSat Design Specification" currently on revision 13 [1] which is maintained by the CubeSat program within California Polytechnic State University San Luis Obispo, define all the information necessary to build a CubeSat to the generally accepted standards. Since the initial CubeSat launch in 2003 [3] the field has seen exponential growth. The option for a less expensive and quicker orbital platform has attracted the attention of far more than the Universities the standard was initially proposed for. As this interest

and the individual capabilities of the CubeSat have increased, the need for additional 


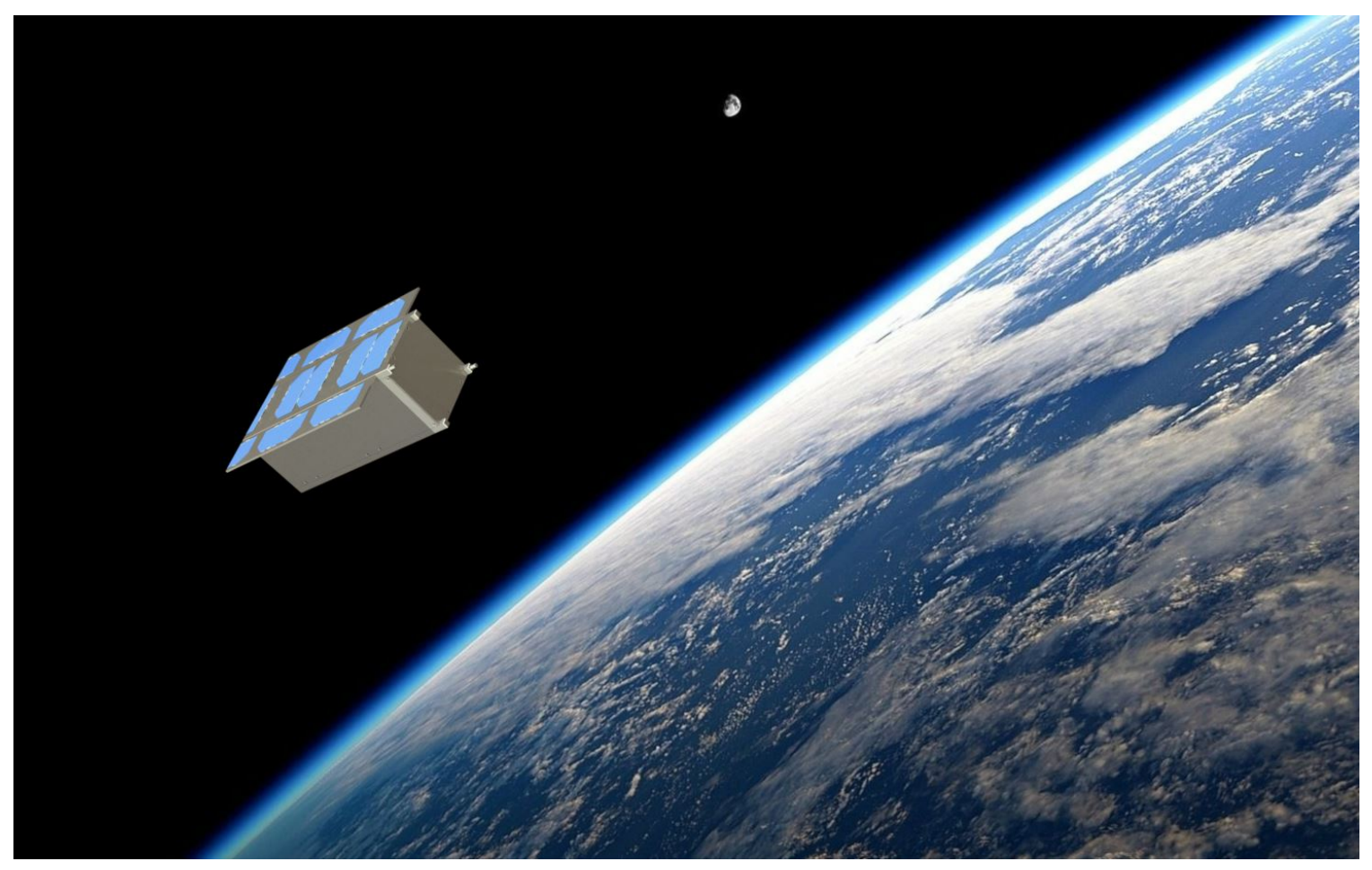

Figure 1.1: Generated Image of Proposed CubeSat in Orbit

capabilities has evolved. The rate of CubeSats needing some form of attitude control has been expanding and is likely to continue to expand with time [4]. Propulsion also enables other desirable capabilities such as orbital control, which includes the need to de-orbit within 25 years [5] to minimize space debris. As of 2010, no CubeSat had launched with propulsion capabilities [6] and as a result the systems where limited to Low Earth Orbits that allowed for passive de-orbiting. This has the effect of limiting potential science missions that CubeSats could feasibly complete.

This need has led to the adapting of old technology and the creation of new technologies for use in CubeSats, such as liquid monopropellants, micro-electro-mechanical systems (MEMs) thruster devices, field-emission electric propulsion, colloid thrusters, and pulsed plasma thrusters for example [6]. Most of these systems are being designed with thrusts well under 1 Newton, [6, 7] likely because of the restriction in available size, weight, and power associated with CubeSats. As the mass of CubeSats is already 
low this means that an effective low SWaP option is within reason for this class of mission. The risk associated with flying an untested propulsion system can make it difficult to find missions willing to risk the use of propulsion systems for testing as a secondary or tertiary scientific mission. Additionally, there are limitations built into the CubeSat standard itself as a means of protecting the hardware launching with it, such as no pyrotechnics and limiting the stored chemical energy to under $360 \mathrm{KJ}$ $[1,7]$. However, this is still one of the most common ways for propulsion systems to get their first flight test. For example, the colloid microthruster ST7-DRS developed conjointly by JPL and NASA Goddard Space Flight Center. Performance results from its 2015 mission on-board the ESA LISA Pathfinder where published in 2017 [8]. The majority of flights have no published results, which leaves the performance of any propulsion system questionable. For example, the Delfi-N3xt CubeSat flew the $\mathrm{T}^{3}-\mu \mathrm{PS}$ which was able to determine that thrust was in fact generated by their cool gas generation thruster, but because of failure in a pressure sensor have no means to actually quantify the produced thrust [9]. However, another path exists, that of the Enpulsion FEEP thruster IFM Nano which flew as a tech demo on-board a 3U CubeSat with the thruster test being the sole mission [10]. The concept of trying to use nano-satellites as a cheap propulsion test-bed is not original. A design from Arizona State University details the design of a cylindrical satellite that intends the thrusters to impart a spin as a means of determining the strength of the thrusters [11]. It does seem like the most feasible way to gather this valuable flight data and increase a propulsion systems technology readiness level for the majority of systems at this point in time.

This thesis will focus on the use of Pocket Rocket, a wave-excited plasma thruster first envisioned by Christine Charles of the Australian National University. It is first referenced in a 2009 paper detailing different possible applications to utilize plasma in a propulsion system [12]. Future work included development of a physical Pocket 
Rocket made and tested at the Australian National University, the tests included firing the thruster into a glass tube with internal pressures varying between 0.3-5 torr with a variety of power input ranging from 5-40 Watts. This information was used to generate a global model of the discharge and to analyze the operating conditions of the thruster [13]. The next iteration saw the Pocket Rocket installed with its various support equipment such as an RF power board and the propellant storage and feed system into a representative 1U CubeSat volume [14]. Stanford formalized the design of the RF power board and reported the output voltage and amperage in conjunction with the Australian National Institute in a paper published in 2018 [15]. Finally, students within the Cal Poly CubeSat Lab (CPCL) within California Polytechnic State University- San Luis Obispo have redesigned the Pocket Rocket Thruster. Two of these newly designed thrusters and their support equipment were installed within a $1 \mathrm{U}$ ground platform. This assembly was then tested and showed the successful firing of both thrusters. The CPCL paper from 2018 details aspects of the design such as the standard operation and published measured thrust at $1 \mathrm{mN}$ and provided details to allow the calculation of propellant mass flow which will be used to baseline the performance for future analysis [16]. A thesis was completed in 2018 by Alec Henken which developed a thermal model of a firing Pocket Rocket. This thermal model was then validated through experimental firings. This undertaking shows the outer aluminum body of Pocket Rocket reaches a temperature of about $315 \mathrm{~K}$ after about 5,000 seconds of operation [17]. This analysis shows that Pocket Rocket will not be a significant source of heat generation within the a spacecraft and will inform the thermal analysis for this mission. 


\subsection{Expected Scope}

This thesis will be limited to analyses that may prove the mission to determine the thruster functionality as unfeasible. A transient thermal model is analyzed to ensure the craft can handle the heat generated by the thruster systems as well as environmental input. It also allows the evaluation of any required cooling systems, and their effect on other analyses. A different analysis performed is the power budget, as the increased power consumption requires greater than typical power generation to function. Another aspect will be the communication systems, as there is a large amount of data generated and will need to be communicated in order to determine the functionality of the thruster. Finally, the dynamics model is analyzed. This is a crucial aspect of this endeavor, as it determines if the proposed means of determining thruster functionality is feasible. Work is performed to enable the completion of the above analysis, but no further. As a result, after the completion of this work, the program is somewhere beyond the Systems Requirement Review (SRR) and still short of the Preliminary Design Review (PDR). 
Chapter 2

\section{HARDWARE CONSIDERATIONS}

A key aspect of any CubeSat mission is the hardware selection. This selection is generally driven by need, availability of the component, and cost. There is also consideration to the size, weight, and power necessary to accommodate the component. This section will highlight the components identified as necessary, what the baseline choice is for a given component, and finally an over arching description of the mission conceptual operations. The intent is to purposefully induce a tumble using the thrusters as torque, then measure the degree of response to determine the functionality of the thrusters. This is similar to the Arizona State mission described in Section 1.3 [11]. The plan to intentionally induce a tumble to the craft influences the necessary hardware strongly, necessitating additional hardware from typical Cal Poly CubeSat Lab (CPCL) missions. This additional hardware directly influences the future analyses that form the brunt of this thesis.

\subsection{Critical Components and Subsystems}

The hardware deemed necessary for the mission largely influences the outcome of the various analyses performed in this effort. Firstly, a flight computer is necessary to control the different systems on board the CubeSat, as a flight computer initiates every other function on the craft. To initiate a tumble, two micro-thrusters are necessary to impose a coupled torque. For the thrusters to perform as intended, control boards may be necessary which will also need to be accommodated inside the CubeSat. Additional volume within the craft will also need to be allocated to allow for propellant storage 
and feed systems. After tumbling, the craft needs to be re-stabilized to a 3-axis controlled pointing law, to accomplish this some attitude control system is necessary as well. A key piece of equipment is the Inertial Measurement Unit (IMU), as it will keep track of the rotation and provide the info necessary to re-stabilize. The IMU is also the main scientific instrument as it will record the rotational speed that will be analyzed to determine the thruster functionality. To ensure the IMU is accurate, angle rate error correction hardware is necessary. The data from this hardware will be used to correct any inertial drift that will occur through operation. This angle rate error correction hardware could be a magnetometer, Sun tracker, Earth tracker, star tracker or some combination of these. A GPS will also be necessary, this will aid in determining times in which downlinking is feasible by recognizing when the position of the craft is within communication range. Knowledge of the position of the craft in correlation to the Julian date can also inform the craft of the location of the sun, and thus be used to calculate rough pointing direction. All of these systems require power to operate, the most likely power storage system utilized by CubeSats is batteries. Batteries require some system to generate power to be stored, for a CubeSat this will generally be accomplished using solar panels. Between solar panels and the batteries storing the power, a charging circuit is necessary. Some flight computers have in built charging circuits for batteries. But if this functionality is lacking, a board to perform this task would be instrumental. Selecting components requires a balance between the minimum acceptable performance and the cost of said hardware.

\section{$2.2 \quad$ Selection Details}

As the intention of this mission is to be built by CPCL, the California Polytechnic State University on campus CubeSat team, the use of CPCL hardware is available. CPCL has developed its own flight computer board. This board has the additional 
benefit of including a charging circuit so a secondary board is unnecessary [18]. CPCL has also developed their own communication board, a UHF transceiver with an Ldipole antenna [18]. Both of the systems have strong flight heritage from previous CPCL missions, and a technology readiness level of 9. CPCL reports the use of both the Spectrolab Ultra Triple Junction solar cells, and the Tenergy Lithium Ion 18650 cells for power generation and storage on their CubeSats [18]. As a result these have been selected as the baseline components for this mission, to align with previous CPCL missions. The thruster being baselined for this effort is another endeavor being undertaken by the CPCL team. The Pocket Rocket an electrothermal plasma microthruster originally developed by the Australian National University. The design has seen continued advancement and improvement and is a prime example of a thruster system that would benefit from a TRL increase. The controller board for Pocket Rocket was developed in a joint effort between Stanford and Australian National University [15]. As these are academic institutions, and not a manufacturing house, it is assumed that the controller boards for this mission will be manufactured by CPCL, to the specifications provided by the other universities.

This mission will require the use of hardware CPCL has not used historically to be successful. Namely attitude control systems, in this case both reaction wheels and a magnetorquer system. The reaction wheel bundle is necessary to stabilize the craft quickly and resume power generation to prevent energy depletion of the craft. The reaction wheel bundle being baselined is the NanoAvionics 4RWO reaction wheel bundle. This bundle was chosen for its low SWaP and its high level of momentum storage necessary to stabilize the craft after a thruster firing. To provide a desaturation mechanism, magnetorquers were identified as the most reasonable option. Any passive system would add complication to the science objective and relying on the use of disturbance torques to be used to stabilize the craft will cost significant time, and may result in the premature saturation of the reaction wheel bundle. The ISIS 
magnetorquer board is being baselined for its reasonable cost and ample documented information. Additionally, the inclusion of the Microstrain 3DM-GX5-25 as the IMU to complete the Attitude Determination and Control System (ADCS). This unit was chosen for its accuracy, and reasonable price point. This system has a built in magnetometer to aid in the mitigation of inertial drift. CPCL has also developed a Sun tracker system which at the time of writing is untested, however, it could prove useful for additional mitigation in the future. A JAVAD TRH-G2 GPS system is being included in the analysis to track position in space, to aid in downlinking and any pointing law.

Table 2.1: Table of Hardware Selections

\begin{tabular}{lccc}
\hline No. & Component Name & CPCL Experience & Cost \\
\hline 1 & CPCL Flight Computer & YES & $\$ 18,000$ \\
2 & CPCL UHF Transceiver & YES & $\$ 18,000$ \\
3 & Spectrolab UTJ Solar Panel & YES & $\$ 8,500$ \\
4 & Tenergy 18650 battery pack & YES & $\$ 60$ \\
5 & Pocket Rocket & YES & $\$ 10,000$ \\
6 & Pocket Rocket Controller Board & NO & $\$ 18,000$ \\
7 & JAVAD TRH-G2 GPS & NO & $\$ 1,950$ \\
8 & NanoAvionics 4RWO & NO & $\$ 17,250$ \\
9 & MicroStrain 3DM-GX5-25 & NO & $\$ 758$ \\
10 & ISIS Magnetorquer Board & NO & $\$ 10,200$ \\
\hline
\end{tabular}

As part of the overall goal of keeping the system within the means of a student run CubeSat team, the cost of the components is heavily taken into consideration as well as being tracked. The expected total hardware cost is about $\$ 102,000$, most of these values are for individual components with a few noteworthy exceptions. For CPCL boards, each mission generally prints and assembles three or four copies at a cost 
of $\$ 6,000$ per copy. For this accounting, it is assumed this mission will manufacture three runs of the flight computer, communication board, and the Pocket Rocket control board as the functionality and final design is dialed in. It is also assumed that 6 of the Tenergy 18650 batteries are purchased, enough to create two battery packs of two, with spares in case of damage or defect. As CPCL is a student research laboratory the cost beyond hardware is extremely minimal, as there is no associated labor cost. It is also import to note that this figure does not include any launch costs associated with the mission.

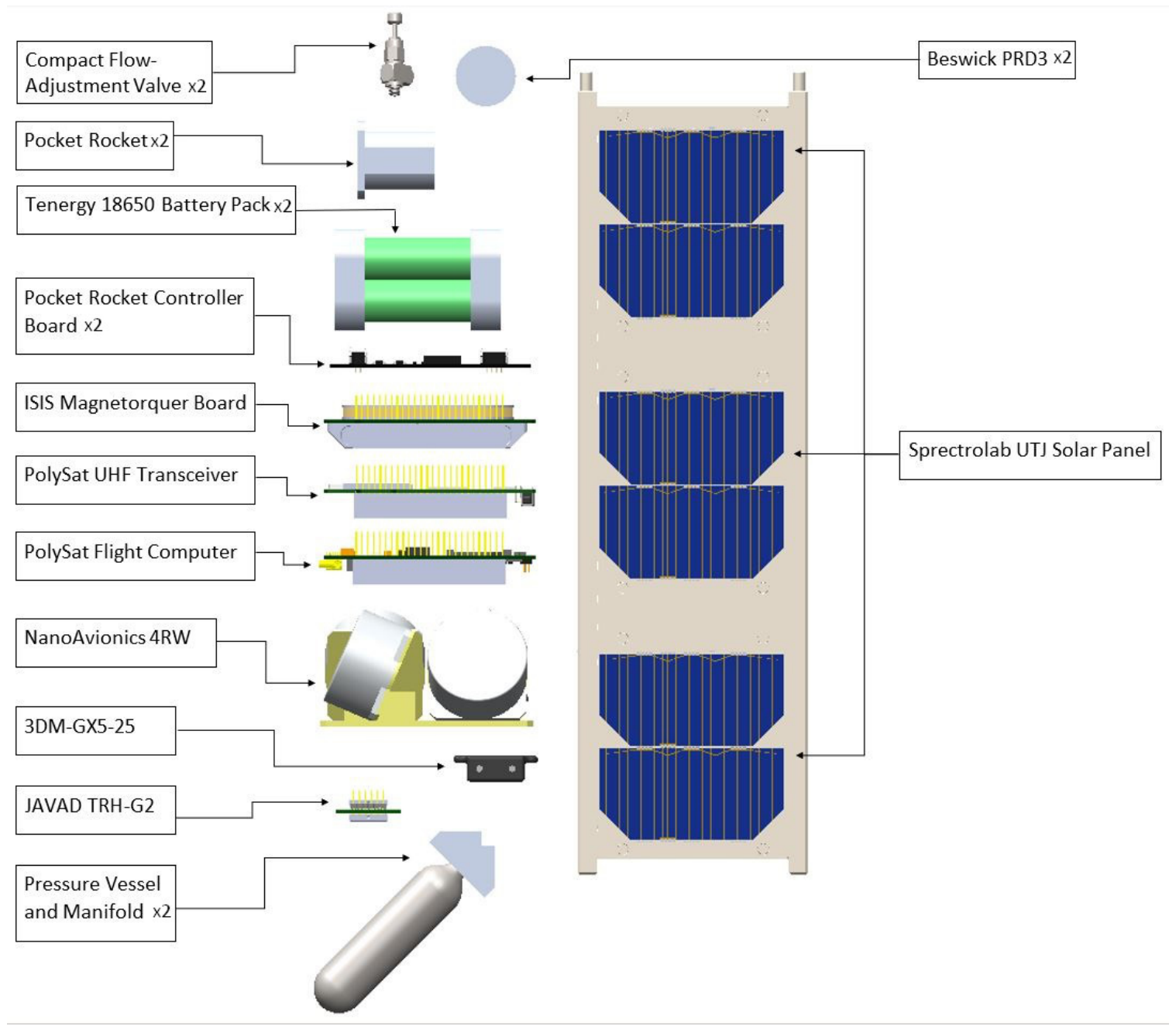

Figure 2.1: Exploded View of CubeSat Design with Component Labels

The exploded view shown in Figure 2.1 does not directly correlate to the layout within 
the assembled craft. The current design is modeled with an effort to evenly distribute mass through the system. At either end of the $3 \mathrm{U}$ volume there is one Pocket Rocket with its storage and feed system as well as one copy of the RF power generation board. Additionally the necessary batteries were split into two individual packs and placed at either end of the craft to minimize their effect on the craft balance. The remainder of the electronics boards listed are grouped together with enough space between each board to add reasonable support structure. Said support structure is not modeled however, as a structural analysis is beyond the scope of this endeavor. The reaction wheel is roughly opposite the electronics board to counter the mass of the grouped boards. Finally the GPS and IMU are placed at roughly the center of the craft, to minimize the effect of a lever arm on the IMU and to keep their effect on the mass balance of the craft.

\subsection{Pocket Rocket Review}

The iteration of Pocket Rocket being baselined for these analyses is the version most recently developed by CPCL and detailed in the paper "CubeSat Electrothermal Plasma Micro-Thruster: System Development and Integration" by Sebastian Gnagy [16]. This Pocket Rocket iteration is constructed from a combination of aluminum, alumina, Macor, and copper. The resultant is a 2.5 centimeter in diameter by 3.5 centimeter in length thruster that weighs $.06 \mathrm{~kg}$. Figure 2.2 shows a cross-sectional image of the CPCL Pocket Rocket [16].

In this system, gas first flows into the plenum. This particular thruster allows for the successful use of multiple gases such as nitrogen, carbon dioxide, and argon. The baseline for both this thesis and the CPCL team is argon. Argon is selected as the baseline propellant due to its lower minimum Paschen pressure and it's higher 


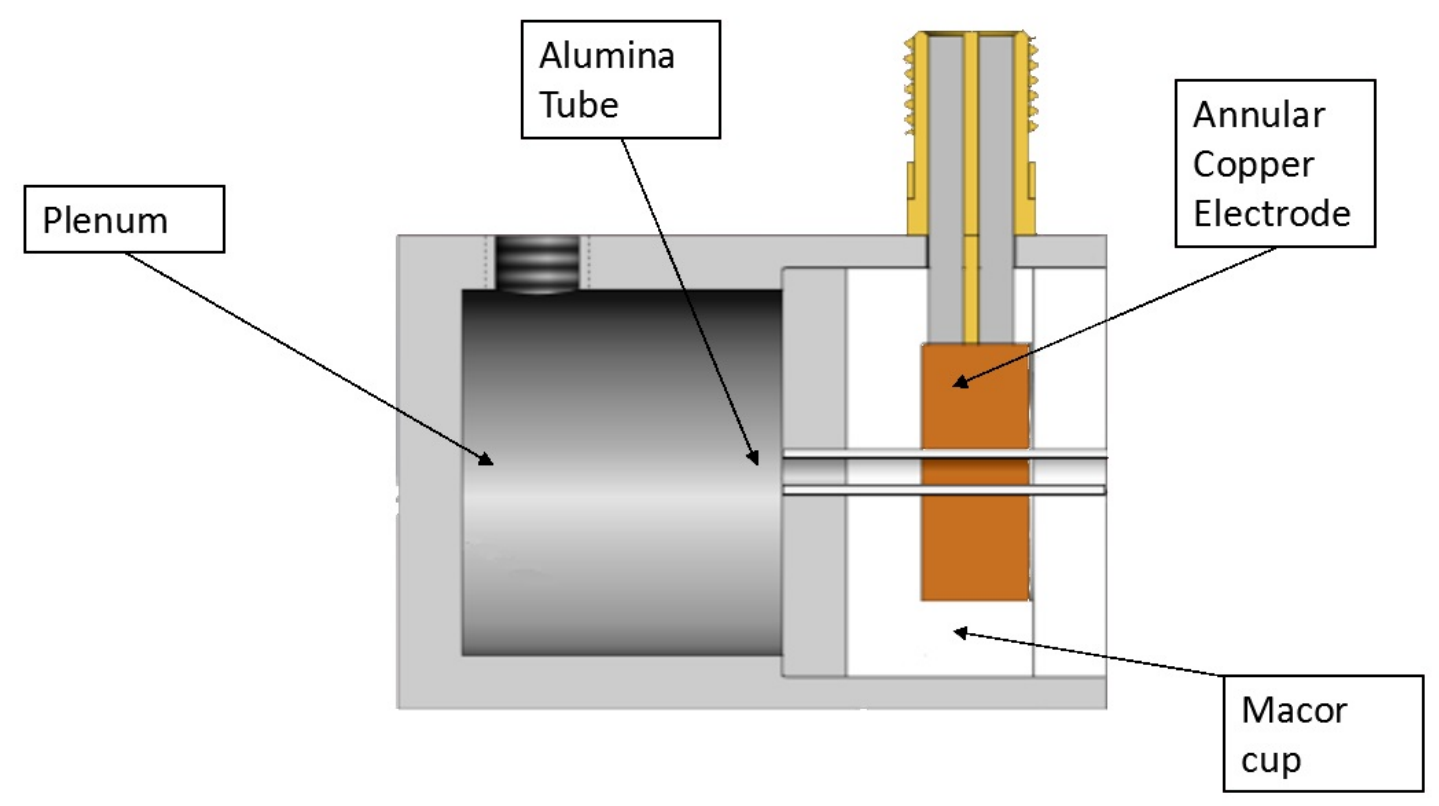

Figure 2.2: Cross Section of the CPCL Pocket Rocket

neutral gas temperature, which results in a higher ISP than other propellant options [19]. Once gas gathers in the plenum, it flows into the alumina tube with an inner diameter of 3 millimeter. As the argon gas travels through this tube it passes through the center of an annular copper electrode. This electrode has RF energy applied to it at $13.56 \mathrm{MHz}$, and as the gas flows through the center this electrode the RF energy is coupled into the gas initiating a plasma breakdown. This results in an extremely high temperature where the plasma is forming, up to 1,000 Kelvin [16]. This extreme heat accelerates the flow, thus improving the attained thrust. The Macor cup acts as a thermal isolator to prevent this heat from spreading to the rest of the thruster body, while also acting as an electrical isolator between the copper electrode and the grounded shell of the thruster.

Another key aspect of the Pocket Rocket detailed in the CPCL paper is the propellant storage and feed system. The same system in which a prefabricated pressure vessel 
with an internal volume of 14,000 cubic millimeters is utilized as the storage tank. This pressure vessel will be connected to a manifold which is slightly modified from the one detailed in the CPCL paper, in that it only holds a single pressure vessel instead of two. This is done as one tank/manifold system will feed each thruster instead of a double tank system feeding two in an effort to simplify routing and ease mass balance concerns. The detailed pressure regulation is also included, to ensure that the flows pressure is properly reduced to Pocket Rockets operational pressure. This setup is duplicated at either end of the $3 \mathrm{U}$ CubeSat so that each thruster has one full storage and feed system full of propellant. The mass flow of this system is calculated to be $1.7 \times 10^{-7}$ grams per second based on the reported run times for the CPCL paper. Assuming the thrusters fire for 20 seconds each test firing, the firing will be limited to 20 seconds as it was discovered firing for 30 seconds could result in more angular momentum than the selected reaction wheel bundle could absorb. If the tanks are pressurized to 0.203 megapascal, the maximum currently allowable by the CubeSat Standard [1]. A total of 540 individual tests can be performed, this represents 36 days of testing if one thruster firing is performed per orbit. More propellant could be added to the system, but this will be the baseline configuration for the following analyses.

\subsection{Proposed CONOPS}

An important aspect of this analysis is the proposal for spacecraft operations post launch. Upon ejection the CubeSat will experience tumble from the ejection mechanism. The on-board reaction wheel bundle will stabilize the craft and then be utilized to enforce a Sun pointing control law. This will allow predictable power generation,

while maximizing the amount of power actually generated. Sun pointing will require less intervention from the Attitude Determination and Control System (ADCS) as 
well, a Nadir pointing law would need to fully rotate the craft 360 degrees about one axis everyday while a Sun pointing law needs to rotate about that same axis 360 degrees over a year. A passive pointing system, a common example is a magnet, will create a persistent force that needs to be overcome during the science experiment. This force will skew the spacecraft's response to the thrusters, thus making results more difficult to discern. An active system will store momentum that will also taint the science objective, but, an on-board magnetorquer has the ability to desaturate the reaction wheels. This effectively eliminates this variable in the post firing analysis. After the wheels are desaturated, the craft will enter an inertial pointing phase during which the CubeSat is ready to perform the science objective.

To test the functionality of the thrusters, two Pocket Rockets are mounted, one at either end of the craft and facing in opposite directions. These opposing thrusters create coupling torques to impose a primarily single axis tumble, this can be seen in Figure 2.3. This tumble will be measurable by gyroscopes which have a higher level of fidelity for less cost than accelerometers that could read the minute transnational acceleration caused by the thruster firing axially. The objective starts with the firing of the two opposing thrusters for a set and known period of time.

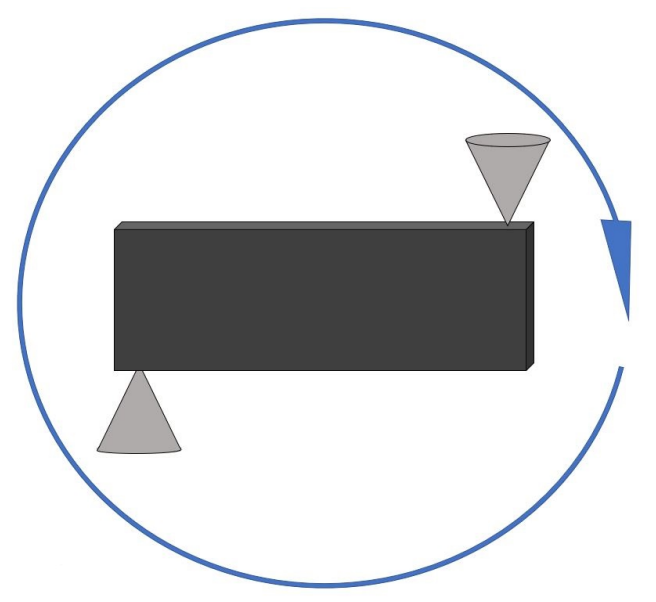

Figure 2.3: Diagram of Coupled Torques Imposing Tumble on Representative CubeSat 
Knowing the time the thruster is fired, the position, alignment of the thruster, and the inertial tensor of the CubeSat allows the force of the thrusters to be calculated from the end tumble rate. Soon after the thrusters fire the on-board reaction wheel bundle will again stabilize the craft, and reinforce the Sun pointing control law. This allows better power generation by the solar panels and time to downlink the IMU data. While this is happening, the magnetorquer will again desaturate the reaction wheel allowing the science experiment to be repeated again to collect more data. The baseline timeline in this analysis has this series of events occurring once per orbit. A notional timeline of the CubeSat mission can be be seen in Figure 2.4.

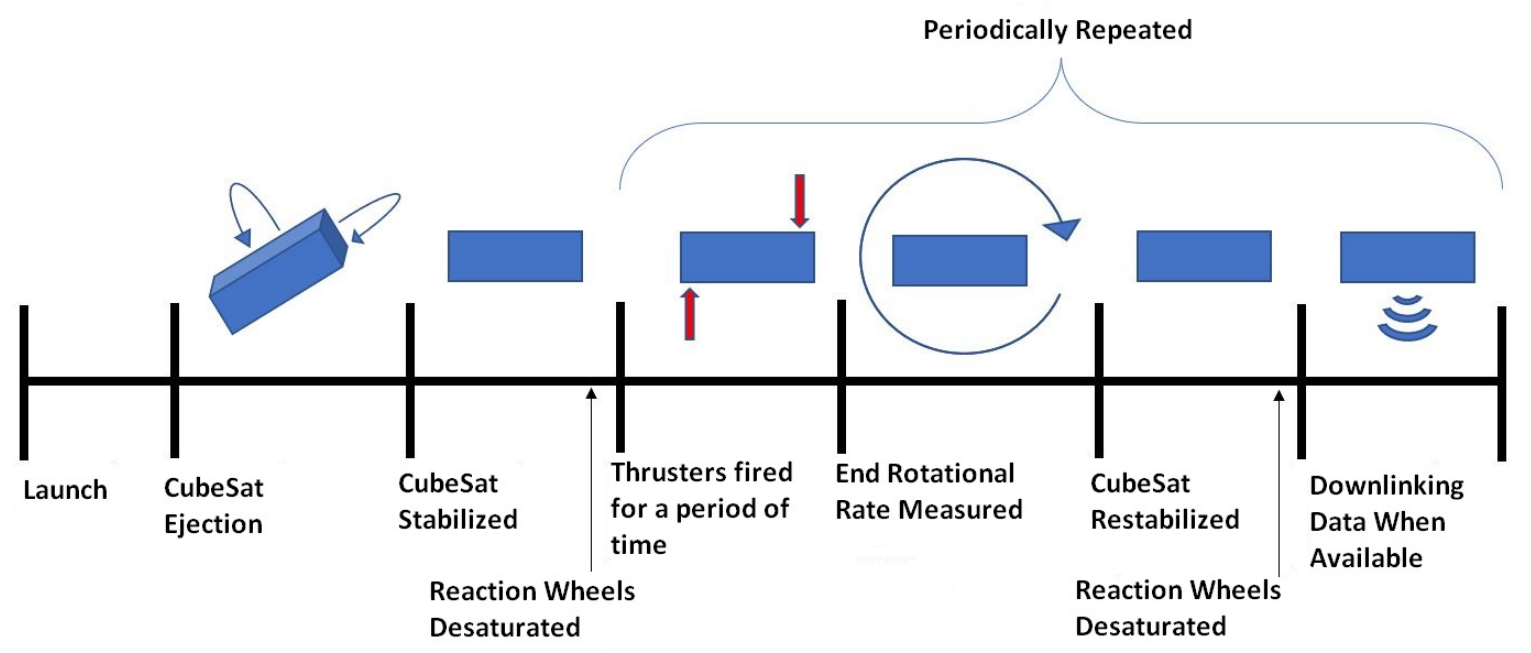

Figure 2.4: Notional Timeline of CubeSat Operations 
Chapter 3

ANALYSIS

Before undertaking the design of a spaceflight mission, a plethora of analyses need to be undertaken to ensure that the mission is feasible and reasonable. These analyses include things like ensuring a power budget closes, ensuring a link budget closes, certifying the craft survives the vibrational environment, evaluating the radiation environment is survivable, and ensuring that radio frequency $(\mathrm{RF})$ and electromagnetic interference (EMI) will not interfere with the internal components. Dynamics are typically considered, however the nature of this particular mission requires a more in depth dynamics model than average due to the intent to induce a dynamic event periodically. The structural, radiation, EMI, and RF analyses are very important aspects of any mission, however, Cal Poly CubeSat Club (CPCL) solving these problems is not as unique as the selected analyses. The goal of this thesis is to discern the feasibility of a mission of this type, as a result effort is focused on the thermal model, power generation, communication budget, and the dynamics model. As these are the analyses that could potentially result in the mission being deemed unfeasible. More detailed descriptions of these analyses can be found below.

\subsection{Introduction and Orbit Selection}

A key aspect in these analyses is the selected orbit for the mission. As the proposed mission's goal is to be executed by the CPCL, the orbit utilized in this effort should be reasonable for a CPCL mission. To ensure this is the case, previous orbits utilized by the team where evaluated. The most common of previous mission orbits had a 
flight height of 510 kilometers at an inclination of 85 degrees. The most recent CPCL Mission ISX utilizes this orbit, and has a published RAAN value of 64 degrees [20]. The orbit for a CubeSat often cannot be chosen, so selecting the most commonly used increases the potential of the selected orbit representing a future flight orbit. As such this orbit is selected as the baseline. To highlight any mission sensitivities and to limit the overall scope of the analysis the orbit is set as perfectly circular with no external perturbations. As the power generation and thermal model are dependant on this selected orbit, any change in this orbit may result in a need to modify the design decisions made. The design selections detailed in further sections included margin to allow a more robust design, thus allowing for adaptability to other potential orbits but large changes in the orbit would warrant reconsideration. Future reevaluations of the analyses will certify the results are still suitable to the mission.

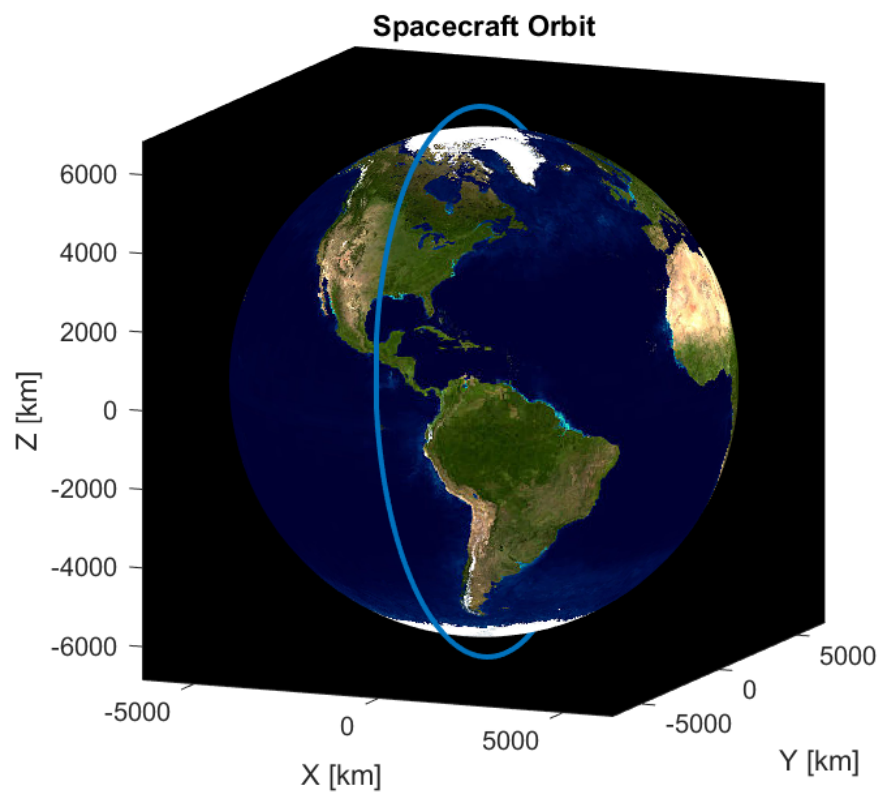

Figure 3.1: Figure Displaying Proposed Orbit of CubeSat 


\subsection{Power Analysis}

A critical component to any mission design is the power budget, as the satellite needs to generate enough power to maintain functionality throughout the expected mission life. The first step is to calculate the power consumption during different modes of operation through the mission. These variable power consumption rates will be dictated by which systems are necessary during these distinct operational environments throughout the mission. The first power consumption mode is standby mode, which is the most common state. It is comprised of the core systems, and also includes the reaction wheel bundle enforcing a control law. The core systems are comprised of the flight computer, GPS, Inertial Measurement Unit, and the transceiver in receive mode. To analyze a worst case scenario the reaction wheel bundle is assumed to be using its full draw potential in this state, 6 Watts. The next mode is the operation of the thrusters, in this mode the core systems are counted as well as the power consumption from two Pocket Rockets. In this state there will be no active control, so the magnetorquer and reaction wheels will draw little to no power, and will last for the 20 seconds mentioned in the CONOPS. After firing the thruster the craft will need to detumble, the core systems will maintain power draw while reaction wheels power consumption is assumed to be the maximum. The selected reaction wheel system would be able to slow down the tumble in less time than the thruster firing, but this period is set at the same length of time as the thruster firing, 20 seconds. This is to add margin for other reaction wheel options in future missions with unknown maximum torques. As a means to ensure the power system is analyzed in a worse case scenario, it is assumed that during both the thruster firing and the detumble there is no power generation by the solar panels. The intended axis of tumble is labeled as $\mathrm{Z}$ in the body reference frame, this axis should be parallel with the sun vector while the Sun pointing control law is in effect. If the tumble is only in the intended axis, 
there would still be power being generated as the craft tumbles however it would be decreasing in magnitude with time. This is due to the incidence angle of the Sun to the panels changing during this time frame. However, if a complex tumble forms then there will be intermittent power generation through this time period. To consider the worst case scenario the model has no power being generated during this time period. After the craft is restabilized the next mode is the desaturation of the reaction wheel bundle. During this time period the reaction wheel power draw will realistically decrease as the magnetorquer allows desaturation, thus slowing the reaction wheels and requiring less power to maintain pointing. To be conservative, the reaction wheel power consumption is assumed to be the full potential draw through the entirety of time necessary to fully desaturate. The time to desaturate the wheels utilizing the magnetorquer is calculated using an average of the Earth's magnetic strength at 500 kilometers, in reality this strength is variable and thus dictates how much torque the magnetorquer can apply. An average allows for an estimate of the time necessary to fully desaturate the reaction wheels, and is found to be about 380 seconds. The wheel desaturating state will occur both before thruster firing as preparation for the science objective and after detumbling as a means to ensure the reaction wheels can maintain pointing before becoming fully saturated. The loss of power generation through two full desaturation cycles as well as thruster firing and detumbling would be a significant burden to the power system, so pointing would need to be maintained through the desaturation procedure. This is feasible, however it would require additional time to desaturate. To accommodate this an additional ten percent of the calculated time is added, resulting in the time to desaturate used in this analysis to be about 420 seconds. The final mode of operation is the downlinking period, this mode includes the same operation detailed in for the standby mode but includes the additional 4 Watts of energy being used to downlink data. This mode can potentially occur during other operational modes, as it is dictated by a position of the craft while this analysis 
determines the operation mode based on time. The results of the power consumption are shown in Table 3.1.

\section{Table 3.1: Table of Power Consumption Rates}

\begin{tabular}{|c|c|c|c|c|c|c|c|}
\hline No. & Component Name & $\begin{array}{c}\text { Power Consumption } \\
\qquad \text { W }\end{array}$ & $\begin{array}{c}\text { Standby Mode } \\
\text { W }\end{array}$ & $\begin{array}{c}\text { Thruster Firing } \\
\text { W }\end{array}$ & $\begin{array}{l}\text { Detumbling } \\
\qquad \mathrm{W}\end{array}$ & $\begin{array}{l}\text { Wheel Desaturating } \\
\qquad \text { W }\end{array}$ & $\begin{array}{c}\text { Communication } \\
\text { W }\end{array}$ \\
\hline 1 & JAVAD TRH-G2 GPS & 1 & 1 & 1 & 1 & 1 & 1 \\
\hline 2 & CPCL Flight Computer & .3 & .3 & .3 & .3 & .3 & .3 \\
\hline 3 & Spectrolab UTJ Solar Panel & - & - & - & - & - & - \\
\hline 4 & Tenergy 18650 battery pack & - & - & - & - & - & - \\
\hline 5 & CPCL UHF Transceiver (receive) & .1 & .1 & .1 & .1 & .1 & .1 \\
\hline 6 & CPCL UHF Transceiver (transmit) & 4 & 0 & 0 & 0 & 0 & 4 \\
\hline 7 & Pocket Rocket & 10 & 0 & 20 & 0 & 0 & 0 \\
\hline 8 & NanoAvionics & $.18-6$ & 6 & .18 & 6 & 6 & 6 \\
\hline 9 & MicroStrain 3DM-GX5-35 & .5 & .5 & .5 & .5 & .5 & .5 \\
\hline 10 & ISIS Magnetorquer Board & 1.2 & 0 & 0 & 0 & 1.2 & 0 \\
\hline & Total & & 7.9 & 22.08 & 7.9 & 9.1 & 11.9 \\
\hline
\end{tabular}

Power Generation by on the onboard solar panels, $P$ (Watts), is predictable by Equation 3.1. This power generation is dependant on the solar flux, $S\left(\mathrm{~W} / \mathrm{m}^{2}\right)$, the efficiency of the panels, Eff (percentage), the area of the panels, $A\left(\mathrm{~m}^{2}\right)$, and the incidence angle of the sun, $\theta$ (degrees).

$$
P=S \times A \times E f f \times \cos (\theta)
$$

Initially a 3 axis controlled Nadir pointing law is analyzed, this enforces one face always pointing towards the ground while another points in the direction of travel. This allows for predictable power generation, as well as some antenna pointing capability. The orbit and attitude of the craft are propagated utilizing ODE45 within Matlab. The control law is a simple PID controller that enforces pointing towards a quaternion with a 20 second settling time and calculates the proportional gain as well as the derivative gain to reorient the craft within the ODE. The initial design had solar panels on the $3 \mathrm{U}$ by $1 \mathrm{U}$ Earth away face and on both the $3 \mathrm{U}$ by $1 \mathrm{U}$ faces 
orthogonal to the Earth away face. These two surfaces will have Pocket Rockets occupying one third of the available surface area, so solar panels will only cover two thirds of the available space. This was found to not generate enough power to close the conservative power budget discussed above. After this discovery the addition of panels on the Earth facing side, and both small faces was considered to see if the conservative power budget could close with a Nadir pointing control law. Unfortunately, the Spectrolab UTJ cells would not be able to generate enough power and as a result the stored energy would steadily decrease until the batteries are depleted. A plot of the solar power generation by all six faces over a year long period can be seen in Figure 3.2. Figure 3.3 shows the power generation on all six faces on the day 300 in Figure 3.2.

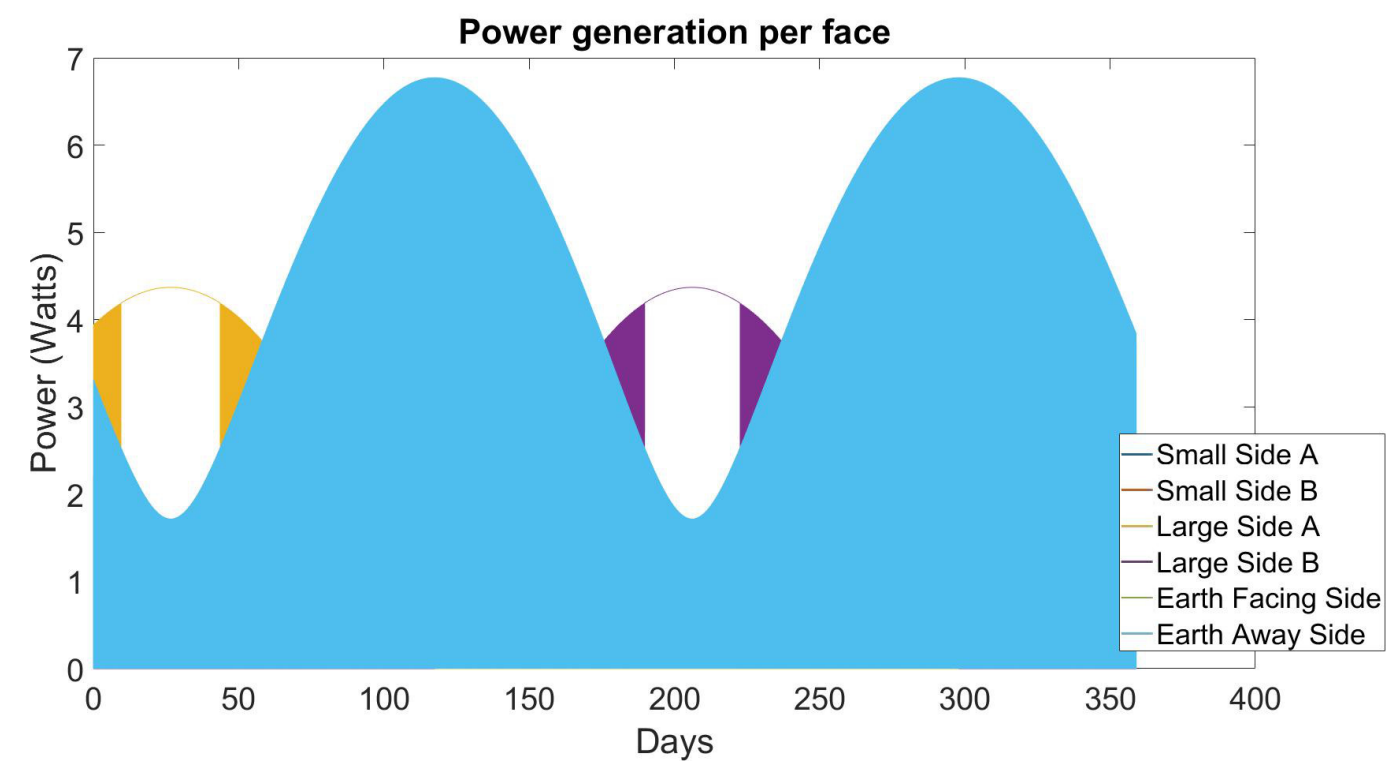

\section{Figure 3.2: Power Generation on All Six Faces with Nadir Pointing}

To aid in the visualization of all future data Figure 3.4 shows the faces corresponding to all future labels and the relative position of the two thrusters. In light of this, analysis is completed to assess the viability of a Sun pointing law. This will provide advantages over Nadir pointing, such as maximizing the amount of power being generated due to the relationship between incidence angle and power generation. Sun 


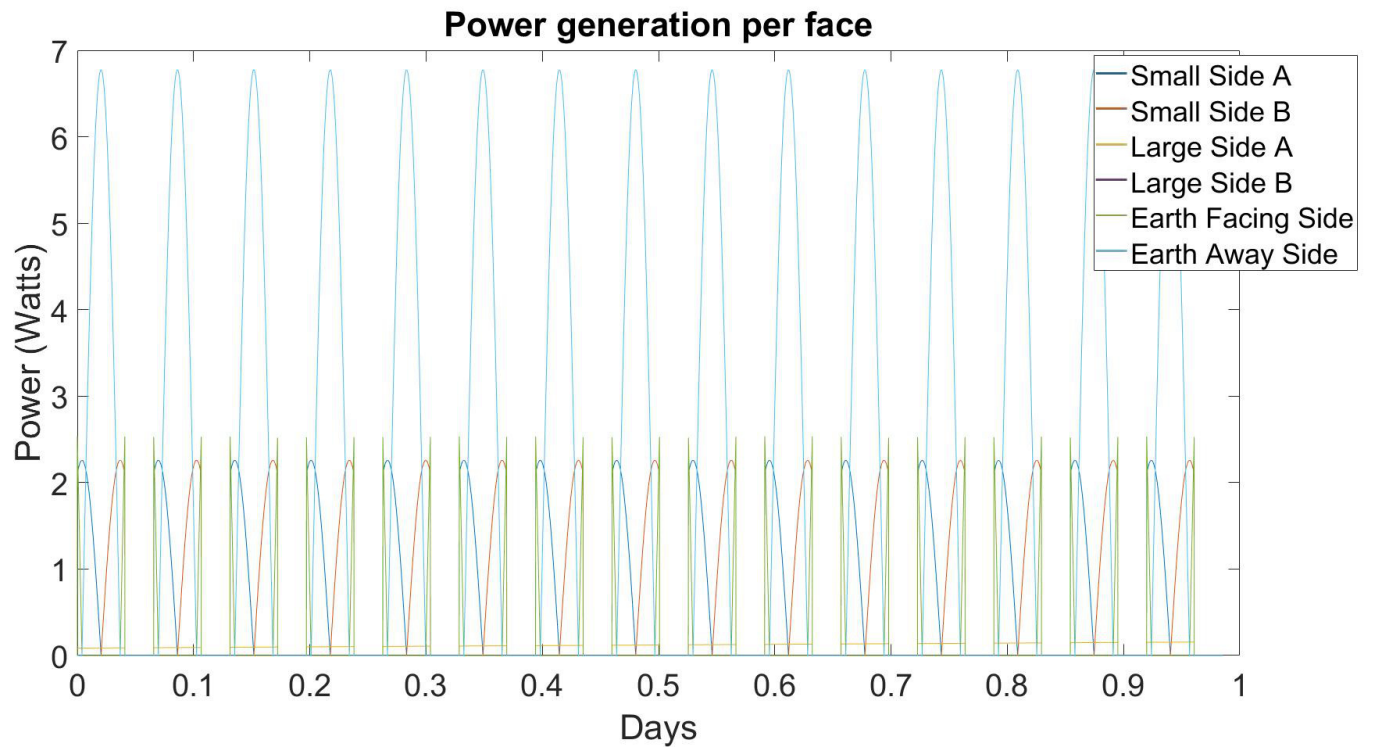

Figure 3.3: Power Generation on All Six Faces with Nadir Pointing on Day 300 in Previous Plot

pointing also minimizes the amount of work the reaction wheel bundle will need to perform, this is because the rate of change to maintain Sun pointing is less than the rate of change necessary to maintain Nadir pointing. The first iteration of the new Sun pointing analysis utilizes only panels on one $1 \mathrm{U} \times 3 \mathrm{U}$ face which is pointed towards the Sun. This results in a constant power generation of 6.9 Watts whenever the CubeSat is illuminated. As the Earth rotates around the Sun this results in periods of constant illumination and times of illumination mixed with eclipse. This can be seen in Figure 3.5. This power generation is less than the 7.9 Watts of power consumption being conservatively estimated in standby mode. If the system uses a less conservative estimate for the reaction wheel power consumption in the standby mode, the power budget can close, but would still have issue with the periods of eclipse. A more reasonable 2 Watts of constant power draw is calculated for the standard use of the reaction wheels. This results in a new standby mode power draw of 3.9 Watts, which is used in evaluating the battery charge for a Sun pointing law with solar panels on only one $3 \mathrm{U}$ face. In this case, the power budget closes when the craft is constantly 


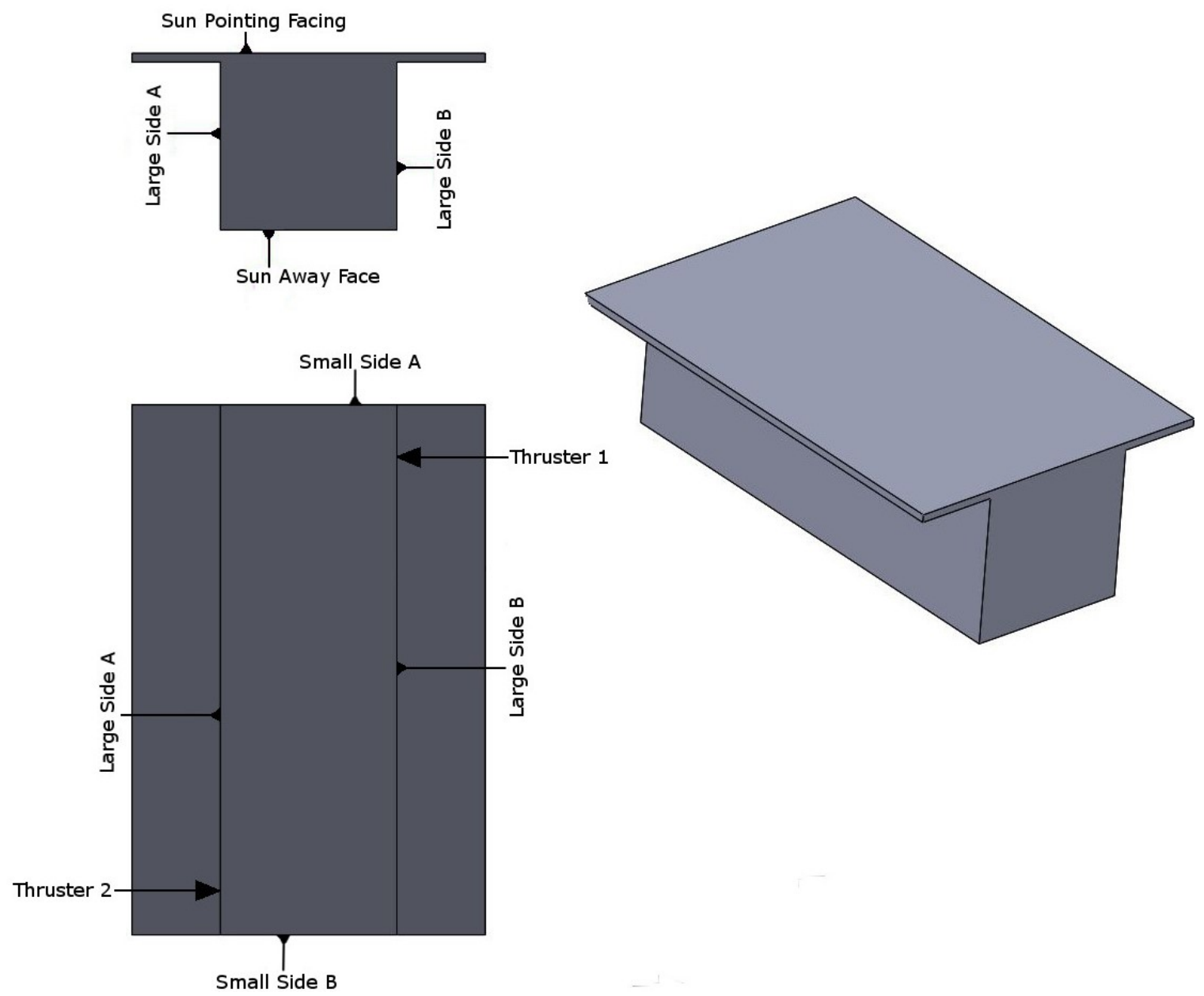

Figure 3.4: Image to Label Sides and Position of Thrusters for Reference

illuminated, but as the craft enters eclipse the power generation cannot keep up with the necessary power consumption. This results in a demand for more power than the batteries contain as seen in Figure 3.7. To overcome this level of power draw, either significantly more batteries would need to be carried on board the craft, or the periodic testing of the thrusters could not occur during the roughly 200 days a year the craft is eclipsing. This could potentially cause other issues with the mission, so an alternative solution is necessary.

CPCL has performed ground experiments with deployable solar panels in the past, this utility would increase the amount of power being generated whenever the craft is 


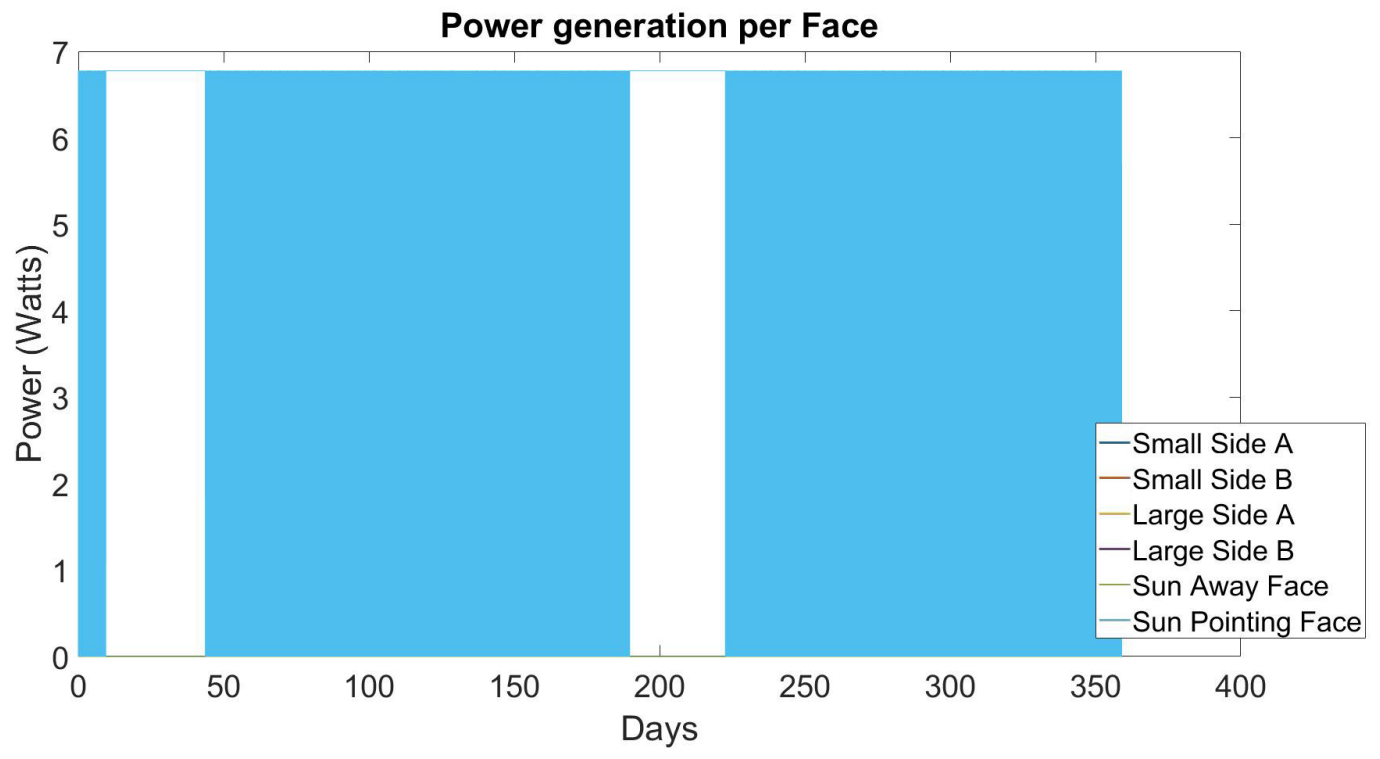

Figure 3.5: Power Generation on Single Face with Sun Pointing

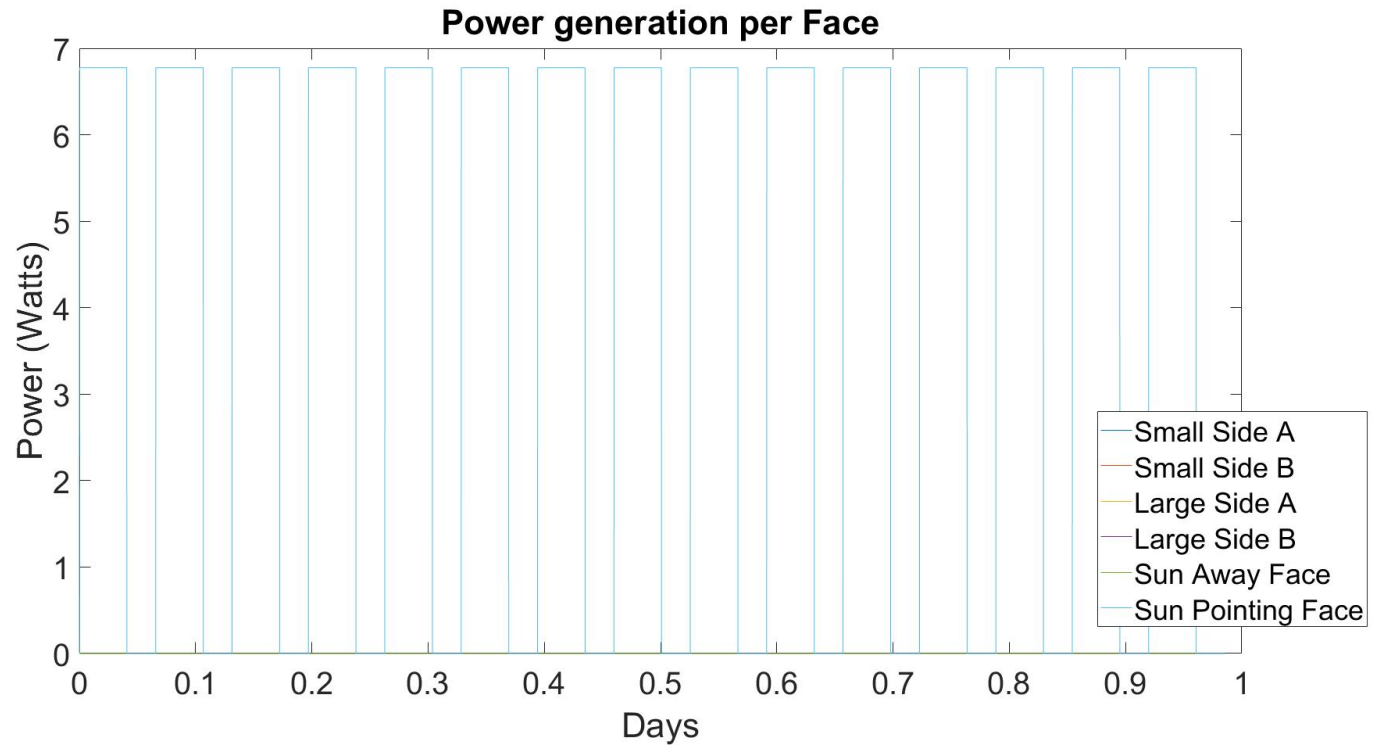

Figure 3.6: Power Generation on Single Face with Sun Pointing on Day 300 of Previous Plot

illuminated. Deployable panels, unfortunately, effect the inertia tensor of the CubeSat. To mitigate the effect on the dynamics model, it is best to use two deployables so the mirrored mass will theoretically cancel out. This will only cause a shift in mass in a non-active axis for the dynamic testing. Minimizing the size of the deployables 


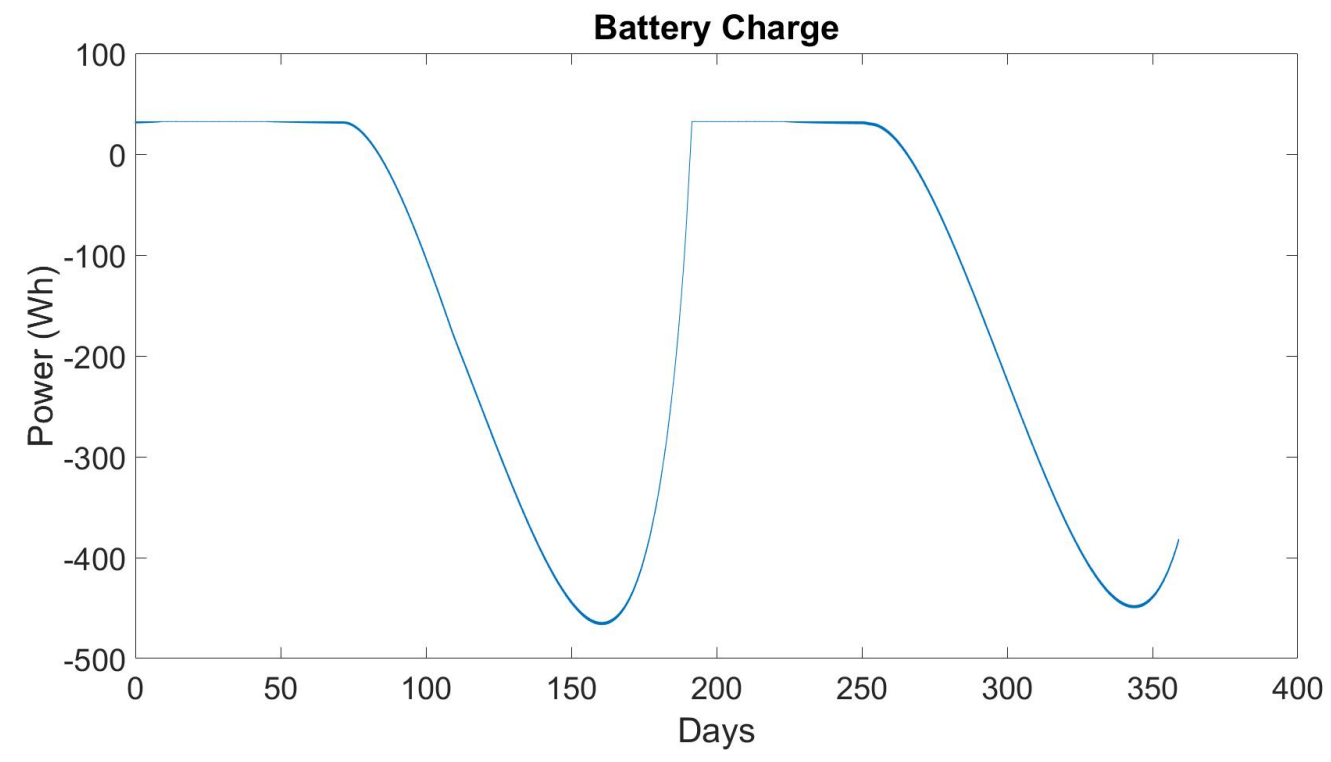

\section{Figure 3.7: Battery Level Using a Single Face with Sun Pointing}

minimizes the risk to the scientific mission. So some effort is invested in reducing the necessary deployable cell area. As a result the baselined design is two deployables that are $1 / 2 \mathrm{U} \times 3 \mathrm{U}$ in addition to the $1 \mathrm{U} \times 3 \mathrm{U}$ surface of the CubeSat. This doubles the area of solar panel being exposed to the Sun, which results in a power generation of about 13.5 Watts whenever the craft is illuminated as seen in Figure 3.8. As a result of this higher power generation, the power budget in this scenario closes with margin as seen in Figure 3.10. The large dips correlate to the period of the year when the satellite is entering and leaving eclipse. The power generation during this time period is represented in Figure 3.9. The batteries will deplete about 17 percent, which is significantly less than the upper limit for the recommended depth of discharge which is eighty percent. This allows for a longer lifetime for the batteries, as well as provide margin for decreasing efficiency as the panels age. Additionally, this margin is small as the two $1 / 2 \mathrm{U}$ X $3 \mathrm{U}$ deployables are realistically more solar panel area than necessary for the mission. This extra area simplifies the mechanical design of the deployables, while increasing the amount of solar panels which mitigates the risk of a panel failing and the resultant power generation being too low. 


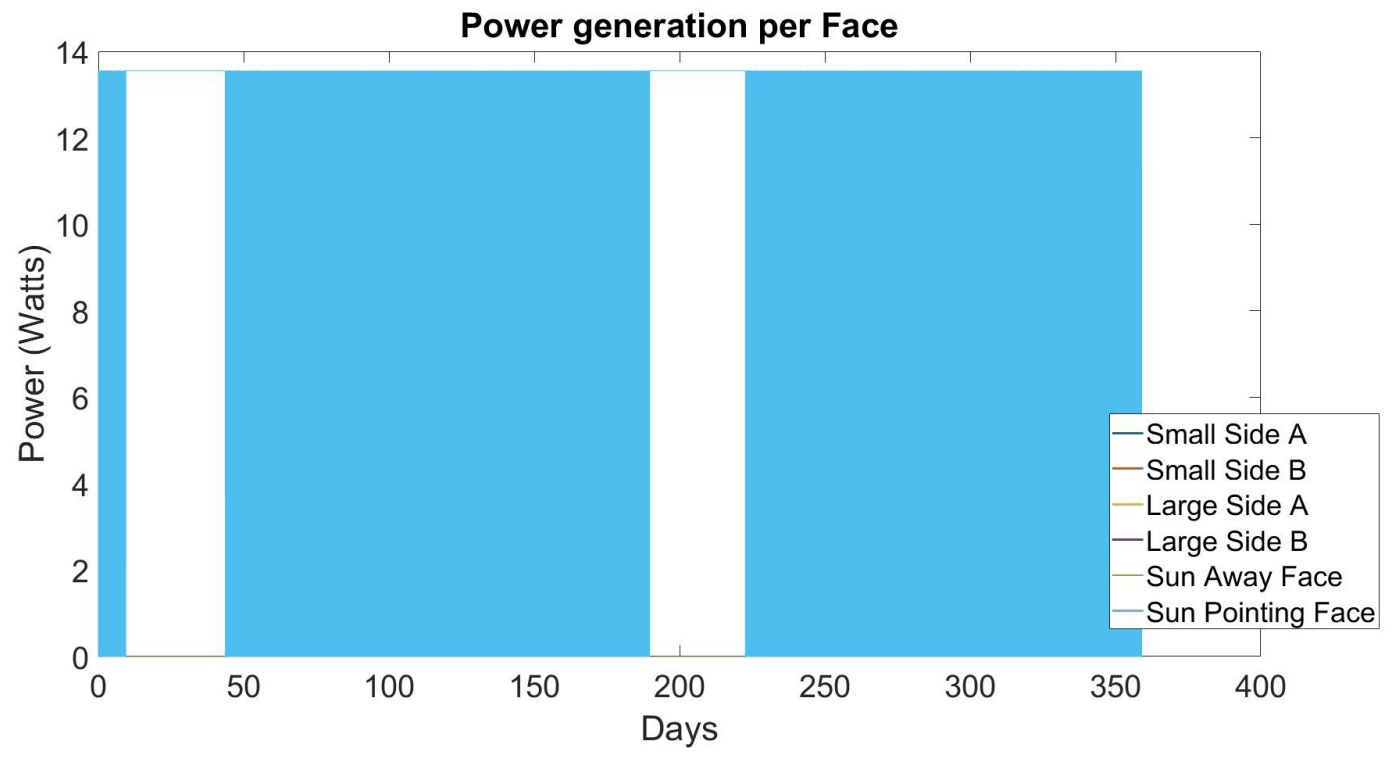

Figure 3.8: Power Generation on Single Face and Deployables with Sun Pointing

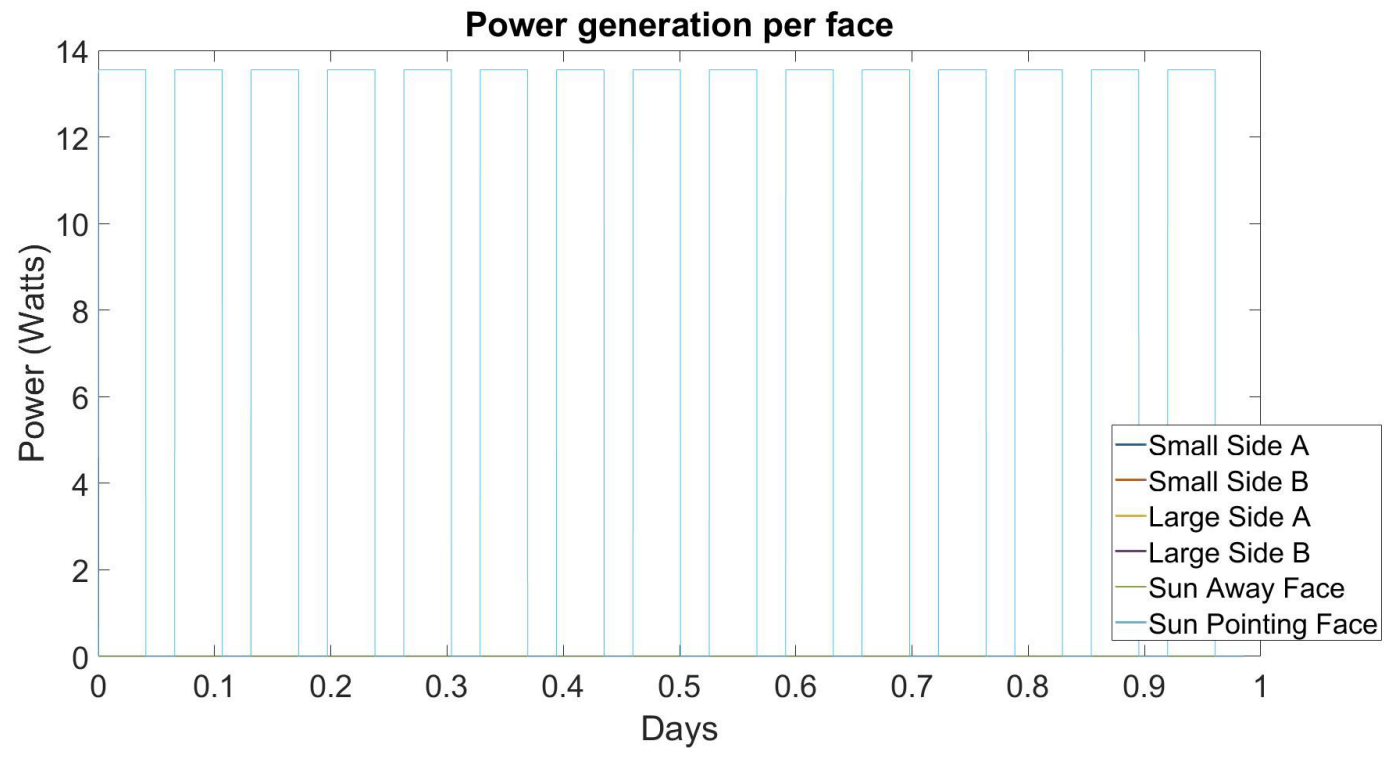

Figure 3.9: Power Generation on Single Face and Deployables with Sun Pointing on Day 300 in Previous Plot

\subsection{Thermal Model}

A transient thermal model for this CubeSat is developed to support mission feasibility assessment. To limit the overall scope and provide a first order feasibility analysis 


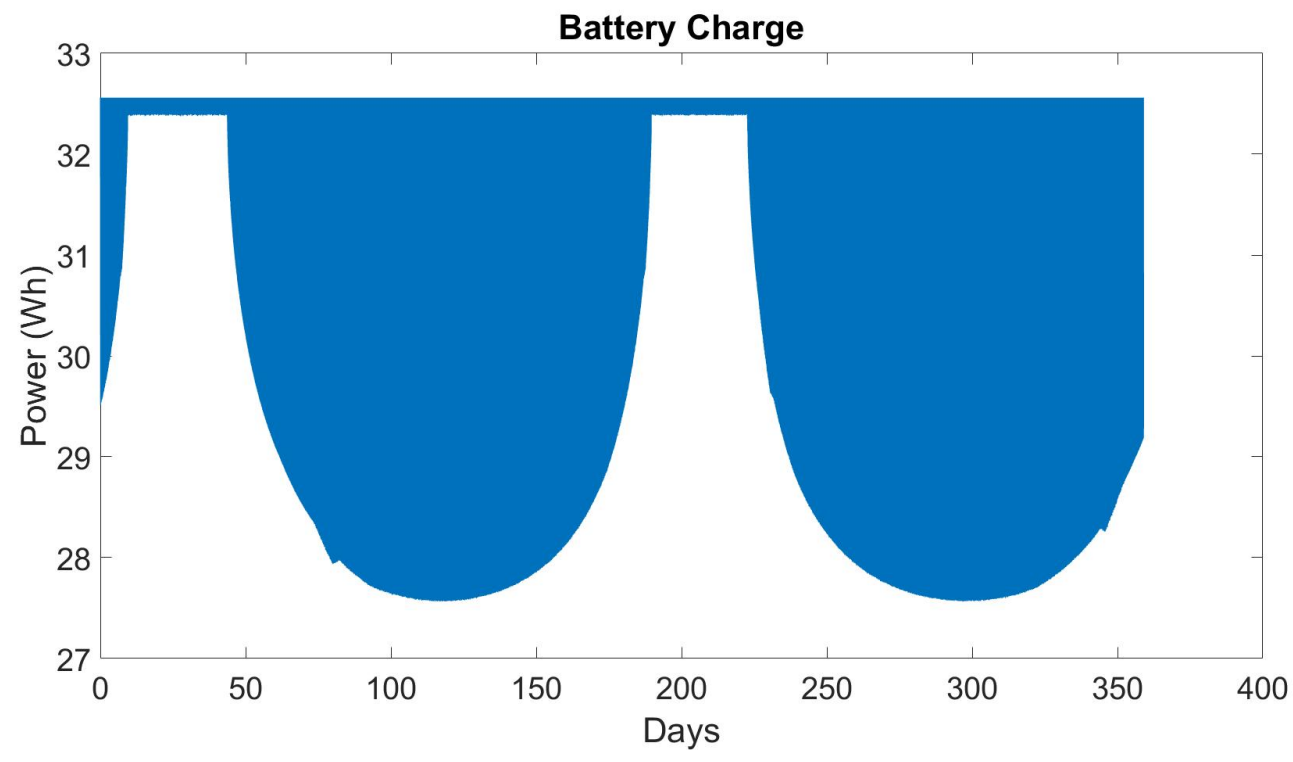

Figure 3.10: Battery Charge for Single Face and Deployables with Sun Pointing

for this effort, a lumped thermal mass method was used. The lumped thermal mass method assumes no temperature gradients exist across the individual faces of the craft or within the internal components, additionally the temperature of the individual components is not modeled but assumed to be within the temperature gradient between the hottest and coolest external faces. A resistance style diagram of the lumped thermal mass can be seen in Figure 3.11. This thermal analysis is intended to highlight the realistic temperatures on all six faces of the CubeSat, and to determine if a SWaP effective passive heat management system can be utilized to keep temperatures within reasonable levels dictated by on-board systems. The transient model analyzes differing environmental conditions and certain periodic events with respect to time. Several external sources of heat will be taken into consideration including direct solar, albedo, and Earth IR. Direct solar is the heat absorbed by the CubeSat from the Sun, albedo is the heat absorbed by the CubeSat from the Sun reflecting off the Earth, while Earth IR is the heat absorbed by the CubeSat from the infrared spectra emitted by the Earth itself. These effects are all largely driven 
by the orbit of the craft with direct solar being the largest driver in the system. Additionally, the heat leaving the craft through radiation to space is largely influenced by the chosen orbit and also considered in the transient model. The model also factors the constant internal heat generation by the on-board electronics as well as the periodic firing of the thrusters and extra power generation associated with stabilizing the craft utilizing reaction wheels. Other periodic events include the desaturation of the reaction wheels, and the downlinking of data.

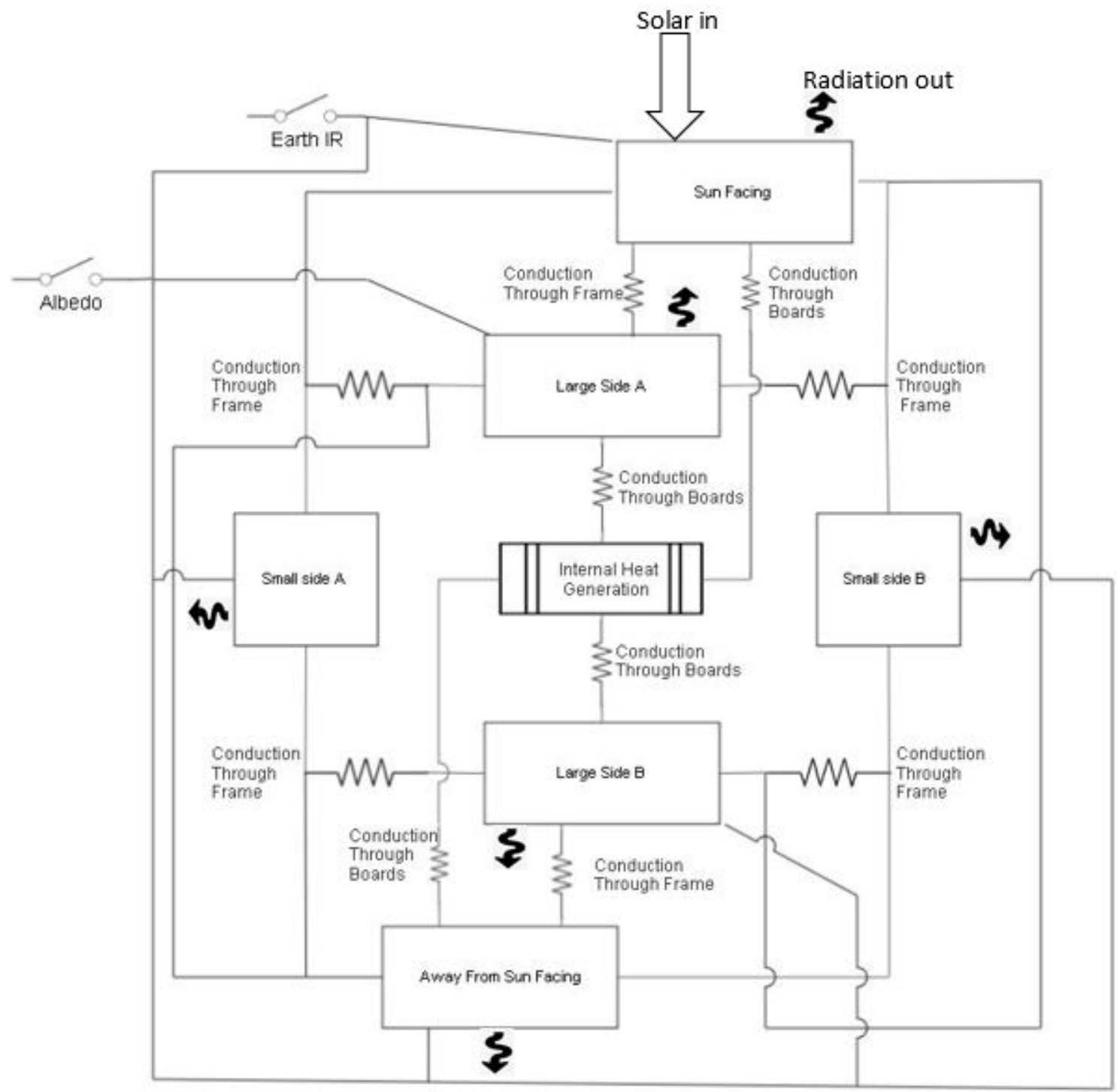

Figure 3.11: Diagram Detailing the Thermal Environment as Resistances 


$$
Q_{s}=\alpha \times I \times A \times \cos (\theta) \times F
$$

Equation 3.2 depicts the direct solar heat, $Q_{s}$ (Watts), where $\alpha\left(\mathrm{W} / \mathrm{m}^{2}\right)$ represents absorption, $I\left(\mathrm{~W} / \mathrm{m}^{2}\right)$ is solar intensity, and $F$ is the view factor. The area of the face illuminated by the sun is $A\left(\mathrm{~m}^{2}\right)$, and the incidence angle of the sun is $\theta$ (degrees).

$$
Q_{A}=\alpha \times A_{b} \times I \times A \times \cos \left(\theta_{A}\right) \times G
$$

Equation 3.3 depicts the albedo heat, $Q_{A}$ (Watts), where $\alpha\left(\mathrm{W} / \mathrm{m}^{2}\right)$ represents absorption, $A_{b}$ is the value for fraction of reflected solar, and $G$ is the view factor. $A_{b}$ varies dependent on whether the satellite is over clouds, the ocean, or land. For calculations in this paper, the value was assumed to be $0.5 \mathrm{~W} / \mathrm{m}^{2}$.

$$
Q_{I R}=\varepsilon_{\text {Craft }} \times \varepsilon_{I R} \times \sigma \times T_{p . b .}^{4} \times A_{s} \times F
$$

Equation 3.4 depicts the direct infrared heating from the Earth, where $\varepsilon_{\text {Craft }}$ and $\varepsilon_{I R}\left(\mathrm{~W} / \mathrm{m}^{2}\right)$ represents emissivity of the spacecraft and Earth respectively, $\sigma$ is the stefan-boltzmann constant, $T_{p . b .}(\mathrm{K})$ is temperature of the planetary body, and $F$ is the view factor.

$$
\frac{d Q}{d t}=k \times A \times \frac{d T}{d t}
$$

Equation 3.5 depicts the conductance equation utilized, where $k$ is the thermal conductivity. The $\mathrm{dQ} / \mathrm{dt}$ term represents the time rate of change of the heat, $\mathrm{Q}$. The $\mathrm{dT} / \mathrm{dt}$ term represents the time rate of change of the temperature, $\mathrm{T}(\mathrm{K}) . \mathrm{A}\left(\mathrm{m}^{2}\right)$ represents the area of the conductance path. 


$$
Q_{\text {out }}=\varepsilon \times \sigma \times T^{4} \times A
$$

Equation 3.6 represents the energy radiated to deep space, $Q_{\text {out }}$ (Watts), where $\varepsilon$ $\left(\mathrm{W} / \mathrm{m}^{2}\right)$ represents emissivity of the spacecraft, $\sigma$ is the stefan-boltzmann constant, $T(\mathrm{~K})$ is temperature of the spacecraft face, and $A\left(\mathrm{~m}^{2}\right)$ represents the area of the craft face.

The majority of components were seen to generate thermal energy equal to their power consumption. A notable exemption, the UHF transceiver which re-routes at least some power back out resulting in less thermal generation. This results in the assumption the UHF transceivers thermal generation is equal to the total power consumption minus the RF power. The power being used by the thrusters and the RF generation board is also assumed to be totally transferred to the craft, although some would realistically leave the craft as plasma. Data for the percentage of power leaving the craft isn't readily available, so this assumption is made to proceed. Table 3.2 contains the acceptable temperature ranges, power consumption, and assumed internal heat generation for the selected components.

\section{Table 3.2: Table of Internal Heat Generation}

\begin{tabular}{lcccc}
\hline No. & Component Name & Thermal Requirement, ${ }^{\circ} \mathrm{C}$ & Power Consumption, W & Heat dissipated, W \\
\hline 1 & JAVAD TRH-G2 GPS & $-40-+80$ & 1 & 1 \\
2 & CPCL Flight Computer & $-30-+85$ & .3 & .3 \\
4 & Tenergy 18650 battery pack (discharge) & $-20-+65$ & - & - \\
5 & Tenergy 18650 battery pack (charge) & $0-+45$ & - & - \\
6 & CPCL UHF Transceiver (receive) & $-30-+85$ & .1 & .1 \\
7 & CPCL UHF Transceiver (transmit) & $-30-+85$ & 4 & 3 \\
8 & Pocket Rocket & - & 10 & 10 \\
9 & NanoAvionics & $-40-+85$ & $.18-6$ & $.18-6$ \\
10 & MicroStrain 3DM-GX5-35 & $-40-+85$ & .5 & .5 \\
11 & ISIS Magnetorquer Board & $-40-+70$ & 1.2 & 1.2 \\
\hline
\end{tabular}


As the systems inside the spacecraft all operate within the same temperature range, the radiation between the electronics within the CubeSat is assumed to be negligible and thus not modeled. Conduction through the PCB material of the internal electronics is considered as well as conduction through the aluminum of the frame. The thruster firing can cause a large influx of heat for a period of time, to avoid damaging the internal electronics the system is designed with excess room to insulate the thruster. This led to the assumption that the thermal energy produced by the thruster firing is conductively transferred to the face of the CubeSat on which it is mounted. From these faces the heat is radiated away or conductively transferred to other faces of the CubeSat, where it is then radiated away. The temperature change on all six faces is calculated at every step through a custom function operating within ODE45, which is also propagating the orbit and attitude of the craft. Within the ODE a PID controller equal to the one described in Section 3.2 is utilized to enforce a Sun pointing law. The absorptivity and emissivity of each face is set individually, one of the six faces is larger to represent the deployable panels and has values in line with the above listed solar panels which have an emissivity of $0.85 \mathrm{~W} / \mathrm{m}^{2}$ and an absorptivity of $0.92 \mathrm{~W} / \mathrm{m}^{2}$. The other five initially have values in line with a bare aluminum finish, an emissivity of $0.09 \mathrm{~W} / \mathrm{m}^{2}$ and an absorptivity of $0.25 \mathrm{~W} / \mathrm{m}^{2}$. The solar panels are exposed to a large amount of thermal energy whenever they are illuminated, a percentage of this thermal energy will be converted into electrical energy and thus does not need to be dissipated by the craft. To simplify and add margin to the model, it is assumed the full solar energy incident to the solar panels needs to be dissipated at all times. This results in a model that underestimates the cold temperature of the craft, as the lower bound has significantly more margin than the upper bounds. This will not pose an issue to the spacecraft performance. Additional spot checking in which the craft is assumed to be constantly charging and thus the percentage of the solar energy being utilized by the solar panels is not added as ther- 
mal energy, results in the lower temperature differing about $10 \mathrm{~K}$ from the model with full thermal energy incidence. The lower bound temperature is realistically between the values shown below and the value minus $10 \mathrm{~K}$, which will not be a problem for any of the selected components. There are two major thermal profiles for a satellite on the selected orbit. One is when the satellite is in constant illumination, and the second is when the plane of the satellite orbit is parallel to the Sun vector resulting in the largest time in eclipse. As the missions time of year for launch is unknown, every analysis begins with an injection into orbit. This leads to the assumption that the initial temperature leaving the rocket will be $300 \mathrm{~K}$, or about room temperature. This is done to see if there is any thermal sensitivity that may dictate launch environment. Figure 3.12 plots the temperature through time when the craft is in constant illumination, while Figure 3.13 plots the temperature as the CubeSat enters and leaves eclipse.

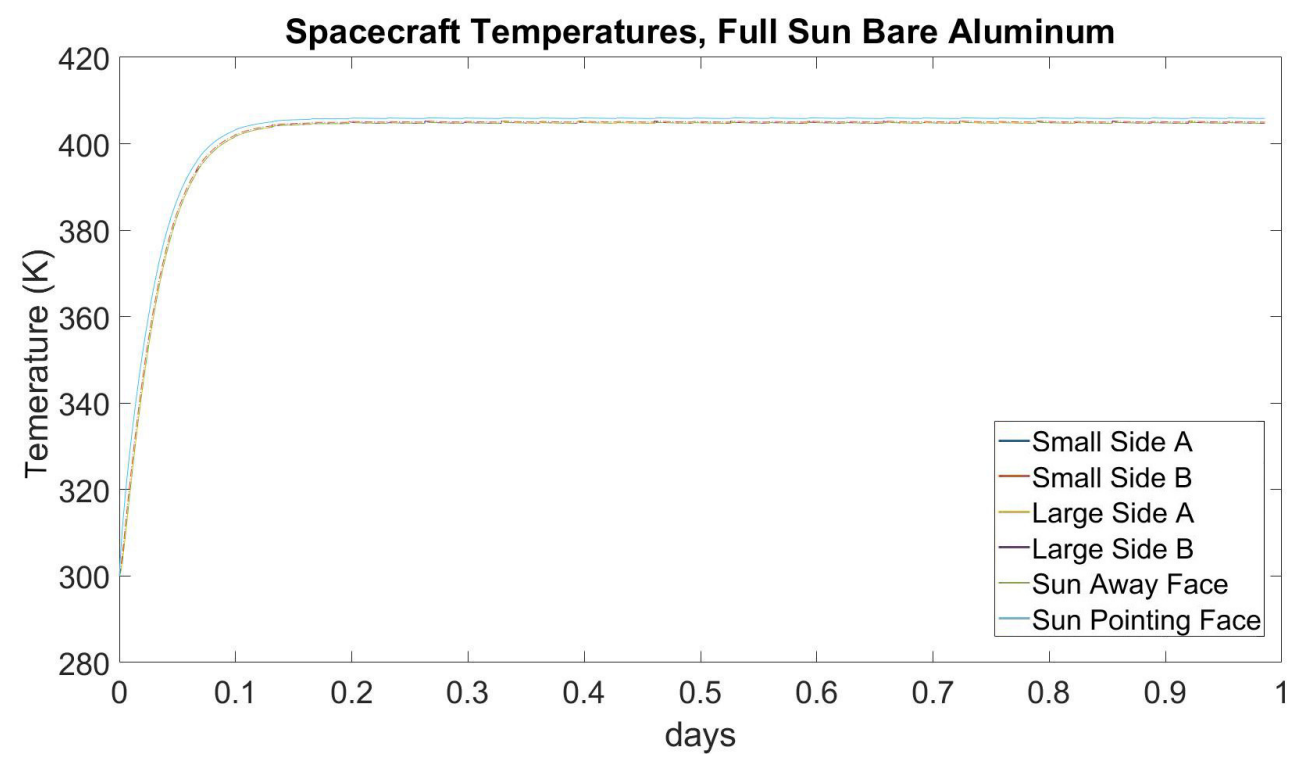

Figure 3.12: Spacecraft Temperatures, Bare Aluminum Shell in Full Sun

In Figure 3.12 the full Sun plot, the CubeSat is ejected at about $300 \mathrm{~K}$ and quickly raises to a relatively steady state. There are temperature variations between faces with a peak temperature of $406 \mathrm{~K}$ or about $133{ }^{\circ} \mathrm{C}$ and a low temperature of $404 \mathrm{~K}$ 


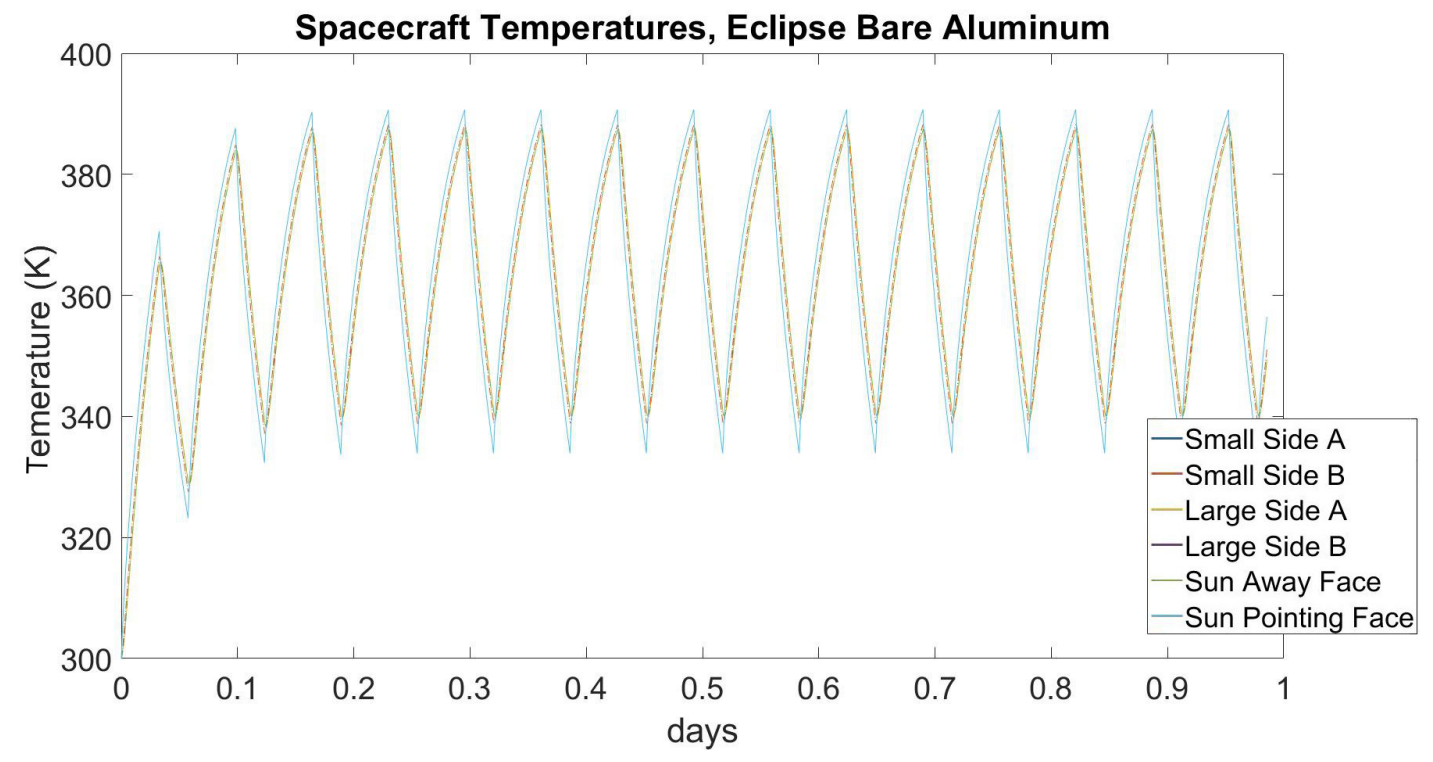

Figure 3.13: Spacecraft Temperatures, Bare Aluminum shell, Eclipsing

or about $131{ }^{\circ} \mathrm{C}$. Slight oscillation shown in the plot lines up with periodic influxes in heat caused by events such as firing the thruster or downlinking data. When the CubeSat is repeatedly entering and exiting eclipse the temperature swing is much more dramatic, peaking at $391 \mathrm{~K}$ or about $118{ }^{\circ} \mathrm{C}$ and bottoming at $334 \mathrm{~K}$ or $61{ }^{\circ} \mathrm{C}$. This decrease occurs as the craft enters eclipse, as it enters the Sun again it quickly reheats. The only means of heat control in this iteration is passive radiation from the solar panels and untreated aluminum shell to dispel thermal energy. The resultant temperatures are greater than listed operating temperatures for all the components chosen for this mission. This means that the system needs modification in some way to be viable for flight. Modifying surface coatings is an extremely SWaP effective thermal management tool. By increasing the amount of thermal energy leaving the system on these faces, the overall temperature of the system can hopefully be lowered to a more acceptable range. The first iteration is an attempt to use a theoretical black paint on all available surfaces to increase the emissivity of that area and because of its high availability. The selected black paint utilizes an absorptivity and emissivity of $0.8[21]$. 


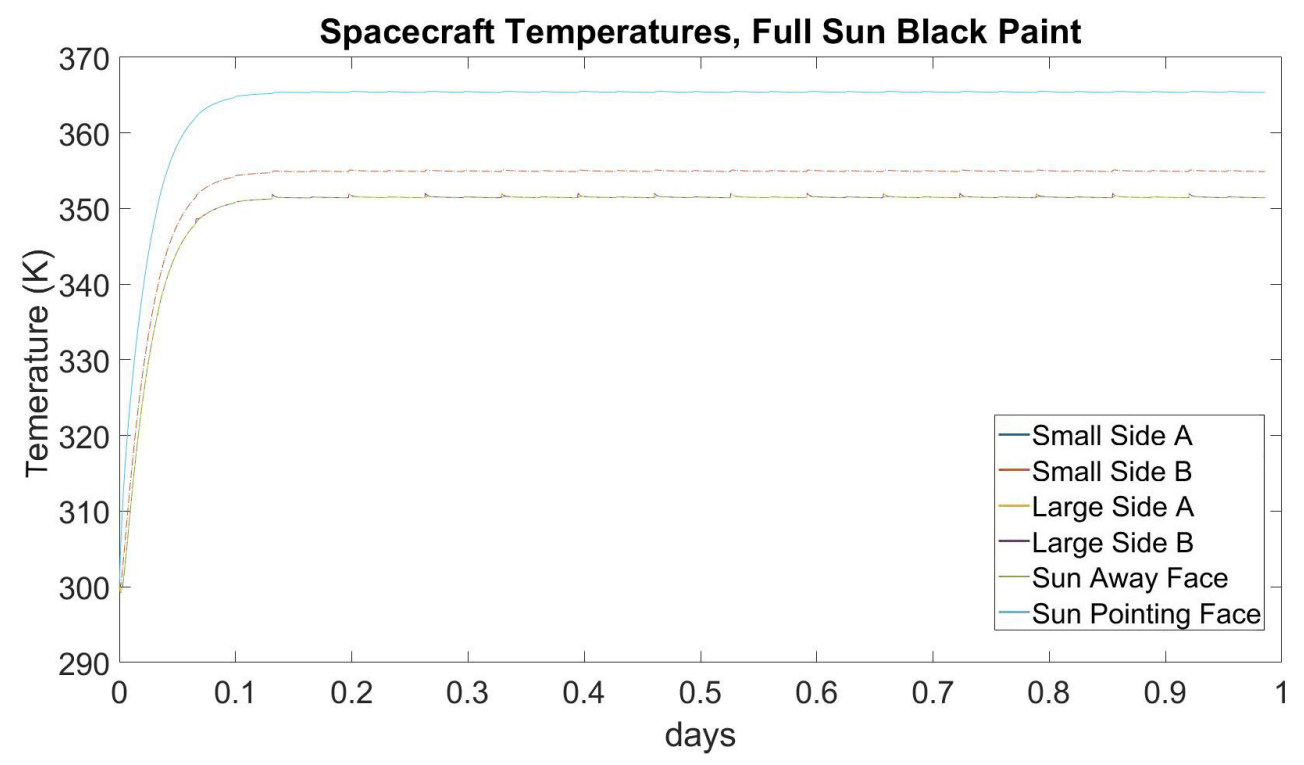

Figure 3.14: Spacecraft Temperatures, Black Paint in Full Sun

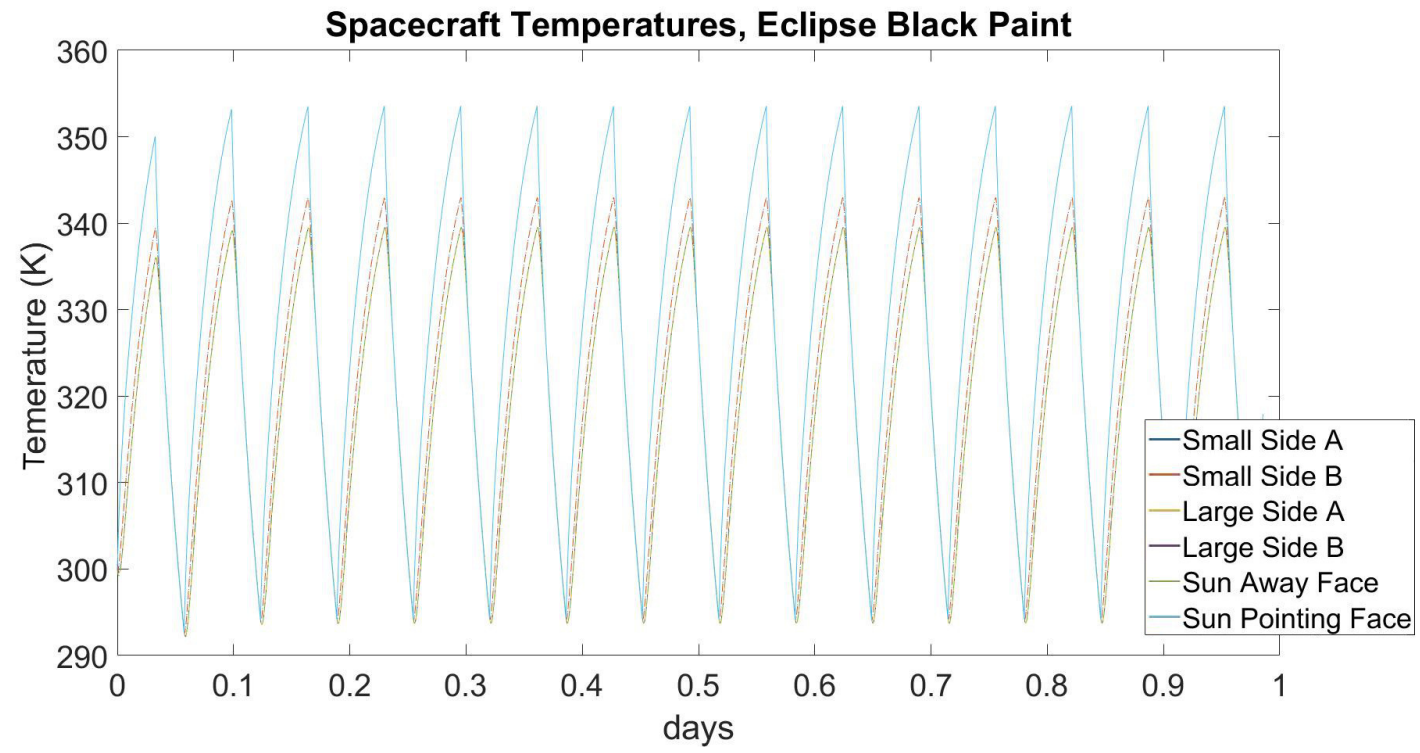

Figure 3.15: Spacecraft Temperatures, Black Paint, Eclipsing

The black paint makes a large difference in the overall temperatures experienced by the craft. In full Sun the temperature gradient between faces peak temperature was lowered to $365 \mathrm{~K}$ or about $92{ }^{\circ} \mathrm{C}$ while the low is now $351 \mathrm{~K}$ or about $78{ }^{\circ} \mathrm{C}$. During the eclipse period the craft sees a temperature swing from $354 \mathrm{~K}$ or $81{ }^{\circ} \mathrm{C}$ to $294 \mathrm{~K}$ or $21{ }^{\circ} \mathrm{C}$. This is an improvement, however $80^{\circ} \mathrm{C}$ is still greater than upper threshold of 
various systems within the craft. The temperature gap between the Sun illuminated face and the rest of the craft has grown, due to the difference in the amount of heat absorbed and expelled by these surfaces. The overall high temperatures are likely the result of more thermal energy entering the system through the faces that do not contain solar panels than necessary. A smaller absorptivity on the non-power generating faces would lessen the total thermal energy entering the craft thus lowering the overall temperature. Maintaining a high emissivity to expel the energy caused by the Sun is necessary, however, too high an emissivity could result in a very large temperature gradient across the craft. Based on this logic a theoretical gray paint was selected.

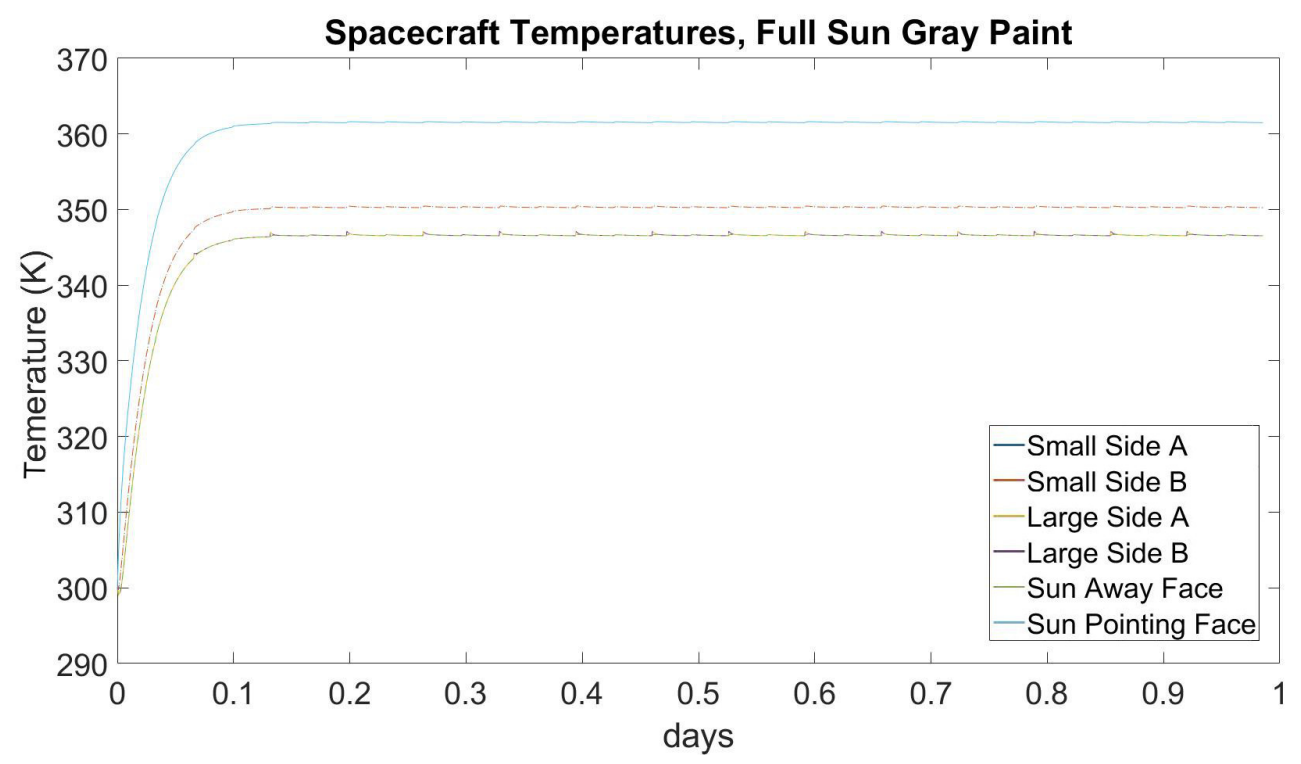

Figure 3.16: Spacecraft Temperatures, Gray Paint, Full Sun

The gray paint selected has an emissivity of 0.9 and an absorptivity of 0.6 [21]. In full Sun the temperature ranges from $362 \mathrm{~K}$ or $89^{\circ} \mathrm{C}$ to $347 \mathrm{~K}$ or $74{ }^{\circ} \mathrm{C}$. While entering and leaving eclipse the craft sees a temperature swing from $350 \mathrm{~K}$ or $77^{\circ} \mathrm{C}$ to 289 $\mathrm{K}$ or $16{ }^{\circ} \mathrm{C}$. In this iteration the faces not pointed towards the Sun now absorb less heat from effects such as albedo and Earth infrared, while the face coated in solar panels is absorbing a high amount of energy from the Sun. This results in a large 


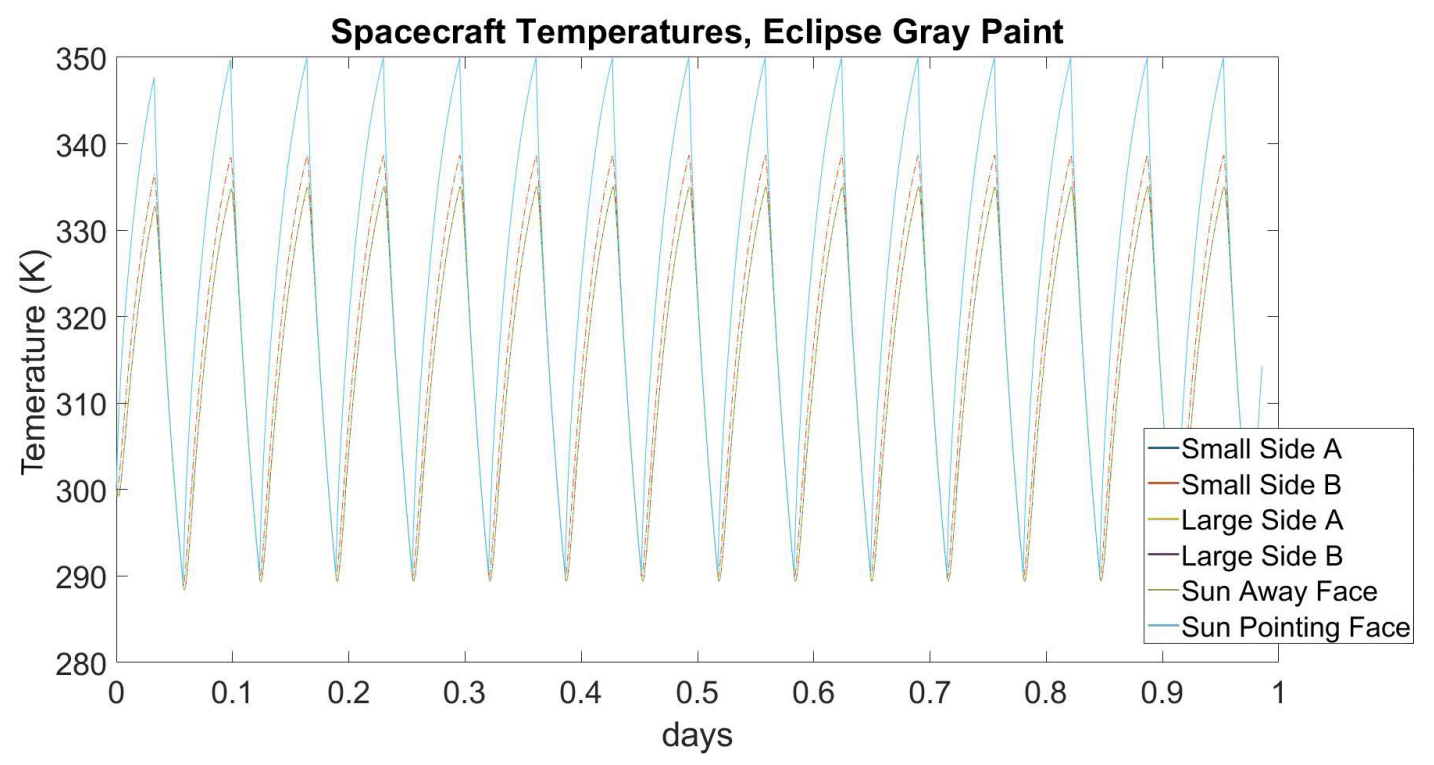

Figure 3.17: Spacecraft Temperatures, Gray Paint, Eclipsing

temperature gradient, which is undesirable. The higher end of this gradient is still too hot, and additionally a thermal gradient of this size could cause structural issues for the craft over time. As the rest of the craft's faces are cooler than the solar paneled faced, increasing the thermal path between all these faces will minimize the thermal gradient between faces of the craft and theoretically be within the acceptable temperature range of all systems.

To accomplish this, thermal straps made of aluminum are added connecting each face to the large face coated in solar panels. This allows increased flow of heat away from the hot face and towards the cooler faces to be radiated away. Aluminum was chosen to represent the mounting structure for all internal components within the craft, as this structure was not being represented in the thermal model previously. As expected, while in full Sun the thermal gradient between faces of the craft is reduced. This results in a high temperature of $317 \mathrm{~K}$ or $44{ }^{\circ} \mathrm{C}$ and a low of $314 \mathrm{~K}$ or about $41{ }^{\circ} \mathrm{C}$. The same effect is noticeable in the plot showing the craft entering and exiting eclipse. Here, the temperature swings from a high of $303 \mathrm{~K}$ or $30{ }^{\circ} \mathrm{C}$ 


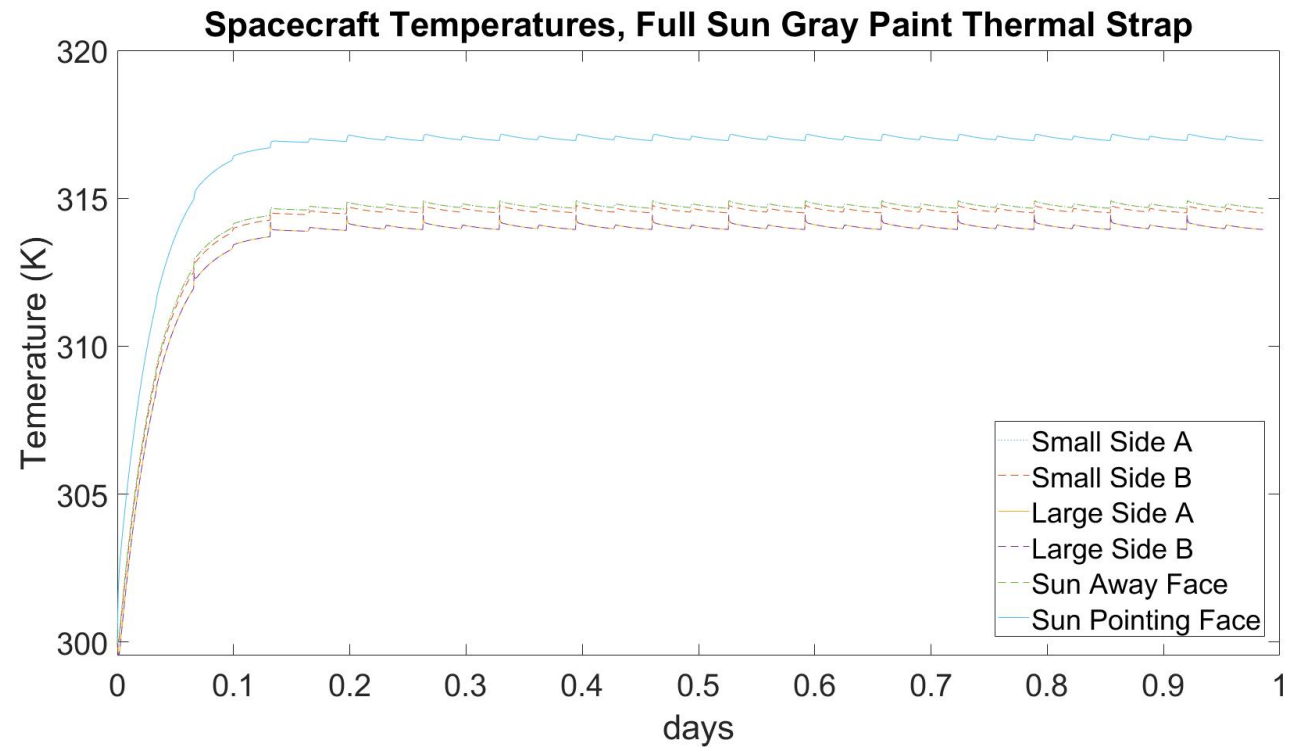

Figure 3.18: Spacecraft Temperatures, Gray Paint \& Thermal Straps, Full Sun

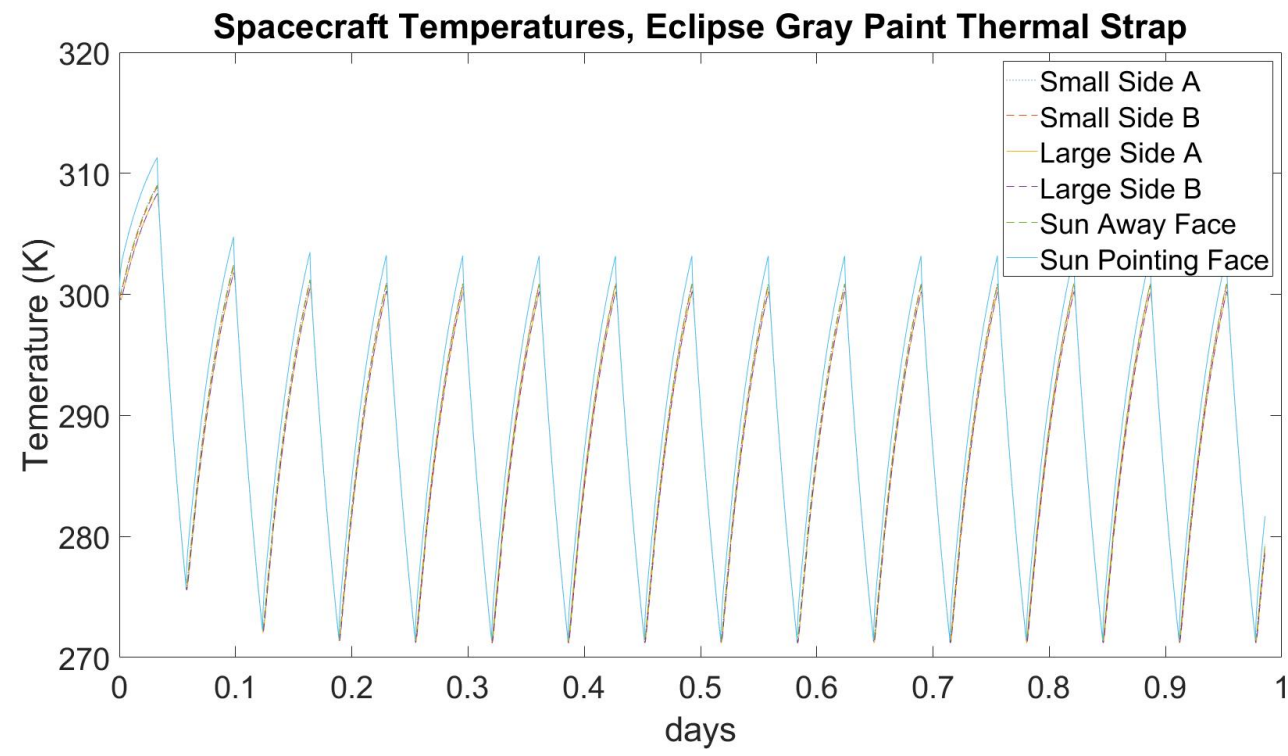

Figure 3.19: Spacecraft Temperatures, Gray Paint \& Thermal Straps, Eclipsing

to $272 \mathrm{~K}$ or about $-1^{\circ} \mathrm{C}$. The thermal straps had an added benefit of reducing the total temperature swing of the craft while entering and exiting eclipse which will allow a reduced wear from thermal cycling. These temperatures are well within the operating range of most systems selected for this mission. The one exception is the 
batteries effective charging temperature range, the craft goes 1 degree too low for this range, which could potentially require a very small heater for the batteries or ensuring the batteries are not charged for a short time after leaving eclipse giving them an opportunity to warm slightly. The upper limit can range from 1 degree to 4 degrees of margin, to ensure the larger margin the batteries are mounted to a face not incident to the Sun. A more detailed future analysis may choose to increase this margin to ensure survivability by modifying the emissivity of the paint and dealing with the even lower temperatures during eclipse with either of the previosuly stated methods. This results in an extremely SWaP conservative method of thermal control, which allows additional SWaP budget for other systems to utilize and additionally will cost less than any active thermal control systems.

\subsection{Communications Budget}

The communication budget for any spacecraft is an important aspect of the mission. It is essential that sufficient data is able to be exchanged between the craft and the ground. In this case, the scientific data that will be analyzed to provide insight to the real world performance of the thruster needs to be downlinked to the ground. The first step requires determining a realistic communication distance achievable with given hardware. The CPCL team uses an omni-directional dipole antenna in conjunction with their communication board. It has a peak gain of $2.15 \mathrm{~dB}[22]$, however, as it is an L-dipole antenna the beam pattern is represented by a roughly toroidal shape. The center of the toroid will have no gain. As the preferred emphasis in the pointing law is power generation and the orientation of the L-dipole within the craft is unknown it assumed to have a gain of $0 \mathrm{~dB}$ at this time. This value is represented by the variable, Gt. If the link budget can close with this assumption, it means that the sun pointing control law will not need to be interrupted to allow downlinking. The 
communication board outputs 1 Watt of RF energy for the 4 Watts it consumes while downlinking[22]. The transmit power is represented by the variable, Pt. California Polytechnic State University has its own ground station, which utilizes M2 436CP42 U/G Circularly Polarized Antennas in a single, dual, and quad Yagi configuration. The single antenna has a gain of $16.8 \mathrm{~dB}$, the dual configuration a gain of $19.5 \mathrm{~dB}$, and the quad Yagi configuration is assumed to have a gain of $22.2 \mathrm{~dB}$ [22]. The gain of the receive antenna is represented by the variable, Gr. The communication occurs in the UHF band, specifically $437-438 \mathrm{MHz}$ so the 0.686 meters is used as the wavelength, $\lambda$. The distance between the satellite and the ground station is dependant on the viewing angle above horizon from the ground station. Given the hilly area around the school ground station, a viewing angle of $15^{\circ}$ above horizon is chosen. With the $510 \mathrm{~km}$ orbit, the $15^{\circ}$ viewing angle results in a communication distance of 1,407 kilometers. The communication distance is represented by the variable $D$ (meters). The bit to noise ratio, $E_{b} / N_{O}$, is found by referencing Figure 3.20 [23]. CPCL utilizes FSK modulation and a probability of bit error of $10^{-5}$ is assumed. The Boltzmann's constant , $\mathrm{Kb}($ Joules $/ \mathrm{K})$, is also considered but converted to $d B W /(H z \times K)$. The data rate which is reported as $9.6 \mathrm{kbps}[18]$ is represented by, $R$.

The System Noise Temperature, $T_{S}$, needs to be calculated using the systems noise figure, $N F$, which is reported as $5.58 \mathrm{~dB}$ by a thesis detailing the development of the CPCL UHF communication system [24]. The noise figure and a reference temperature, $T_{R}$, which is equal to $290 \mathrm{~K}$ are used in Equation 3.7 to calculate the System Noise Temperature/With all this information, Equation 3.8 can be used to calculate the margin in the communication budget $(\mathrm{dB})$.

$$
N F=10 \times \log _{10}\left(T_{S} / T_{R}+1\right)
$$




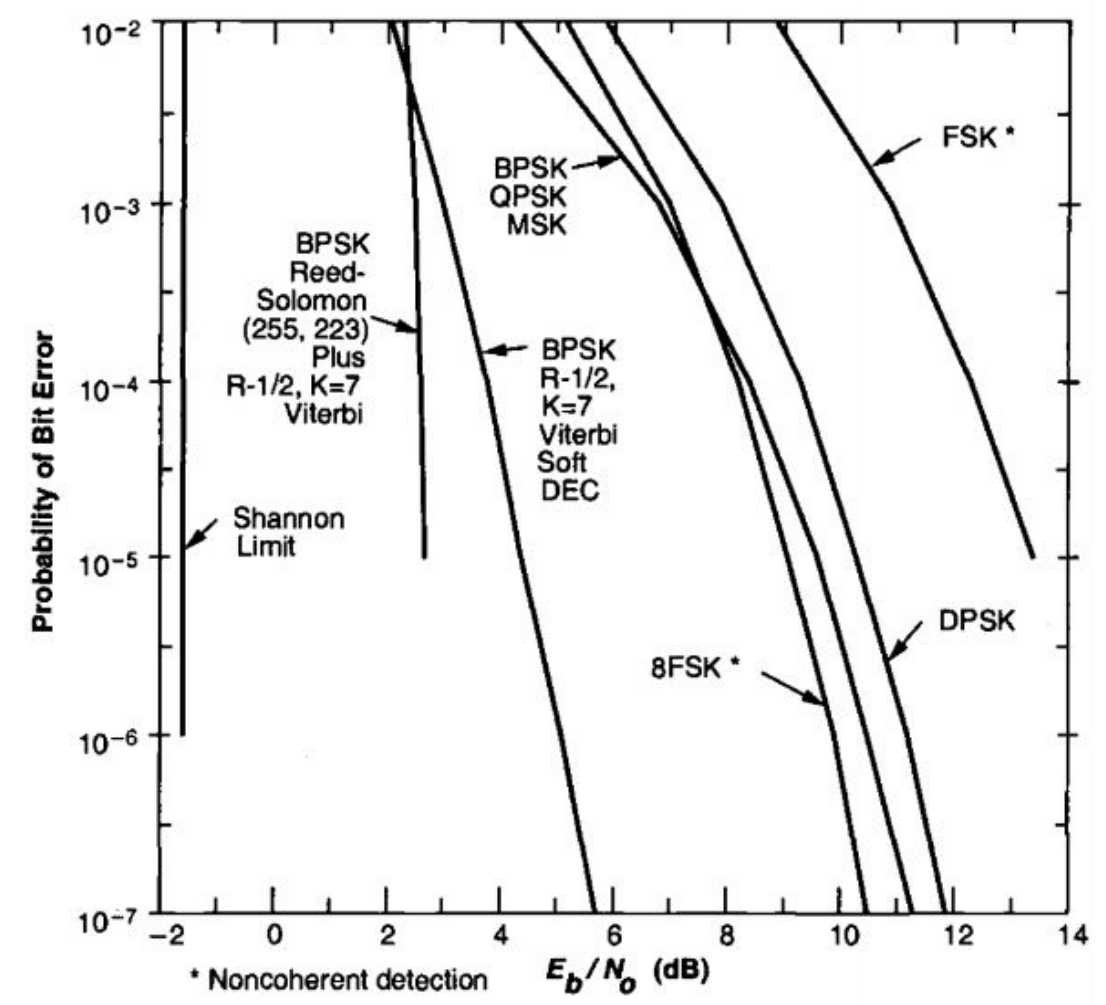

Figure 3.20: Bit Error Probability as a Function of $E_{b} / N_{O}$

$$
\begin{gathered}
\text { Margin }=10 \times \log _{10}(P t)+G t+G r+20 \times \log _{10}(\lambda)-20 \times \log _{10}(4 \times \pi) \\
-20 \times \log _{10}(D)-E_{b} / N_{O}-10 \times \log _{10}(K b)-10 \times \log _{10}(R)-10 \times \log _{10}\left(T_{S}\right)
\end{gathered}
$$

To calculate the uplink margin the same equation is used, however, the gain values are switched for transmit and receive and the transmit power is 100 Watts [24]. The calculated uplink and downlink margins vary depending on which configuration of ground antenna is used. The downlink margins are smaller, because of the smaller transmit power from the craft. The smallest downlink margin is the downlink to the single antenna at $15.5 \mathrm{~dB}$ of margin, while the largest is the downlink to the quad Yagi configuration with $20.9 \mathrm{~dB}$ of margin. Both the uplink and downlink margins in comparison to the antenna configuration are found in Table 3.3.

The time the spacecraft is within the calculated communication window is a key 
Table 3.3: Table of Uplink and Downlink Margin Compared to Antenna Gain

\begin{tabular}{lccc} 
Antenna & Gain dB & Uplink Margin dB & Downlink Margin dB \\
Single & 16.8 & 35.5 & 15.5 \\
Dual & 19.5 & 38.2 & 18.2 \\
Quad & 22.2 & 40.9 & 20.9 \\
\hline
\end{tabular}

factor, that dictates the amount of data capable of being downlinked. The ground station at California Polytechnic State university is at $-120.6625^{\circ}$ longitude and $35.3050^{\circ}$ latitude. From this position, a square communication window is calculated with a diagonal distance of 1,407 kilometers from the center to each corner equal to the distance utilized while calculating margin in Equation 3.8. This bounding bos represents the craft being visable from $15^{\circ}$ above one horizon to $90^{\circ}$ above the same horizon before going back down to $15^{\circ}$ above the opposite horizon. The ground track is plotted by first calculating the orbit through a 24 hour period utilizing ODE45 within Matlab. The circular orbit is then calculated to a 2D representation and then plotted. Every orbit the plot shifts laterally, as expected, as the earth rotates on itself while the the craft orbits it. As this data is being calculated, every time the craft enters a rectangular communication window a counter tracks for how long. As this code is run for a period of one day, the results shows that the CubeSat is within communication range 2 times a day for a total of 12 minutes daily. Below in Figure 3.21 , there is a 2 dimensional representation of the crafts ground track over a 24 hour period with the communication window being represented by a box of stars.

The CPCL team states the expected data rate for this setup is $9.6 \mathrm{kbps}$ [18], if this rate is met for 18 minutes per day that means a total of 6.912 Megabits per 


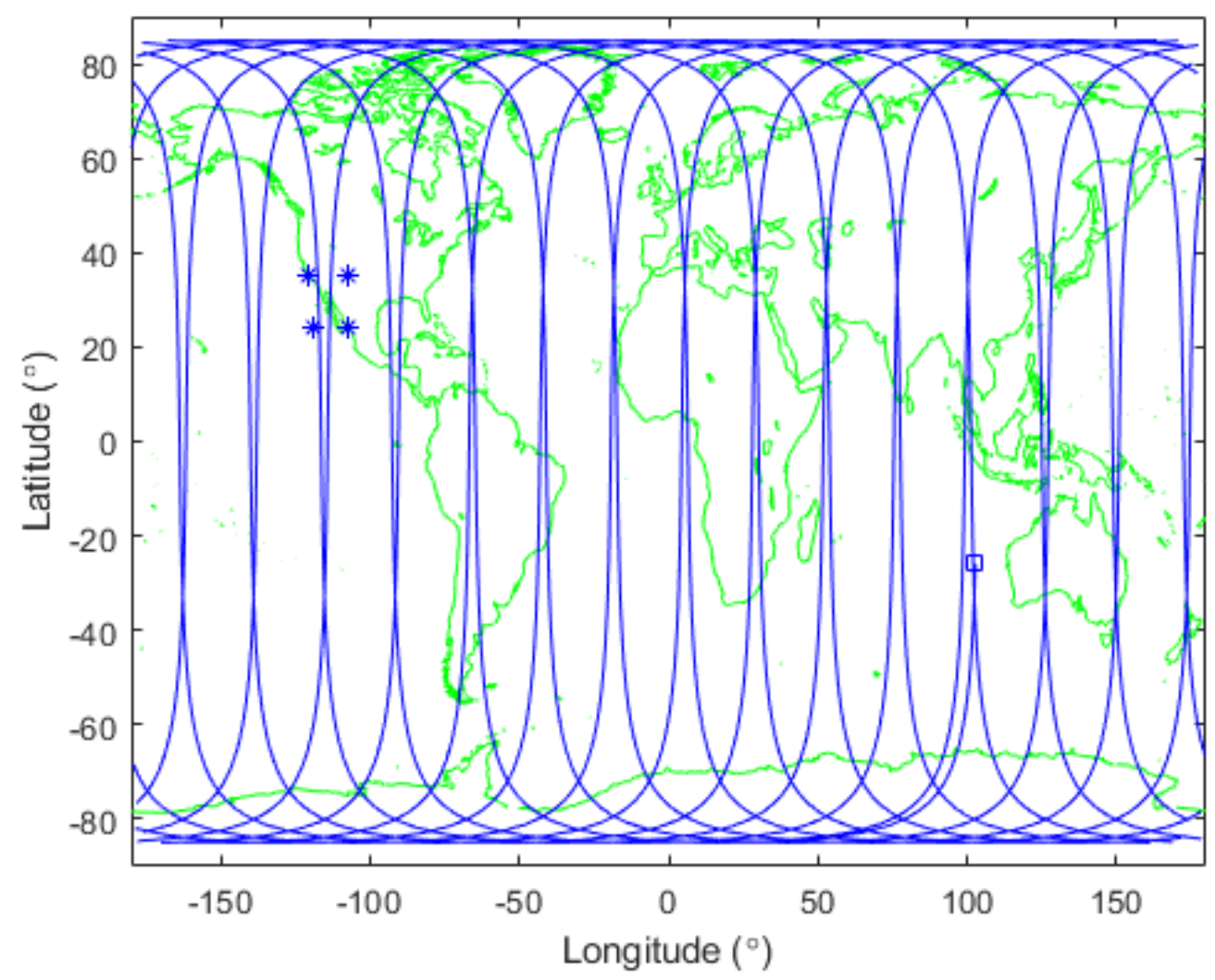

Figure 3.21: Spacecraft Groundtrack, Communication Window Represented by Star Square

day can be downlinked. The Microstrain 3DM-GX5-25 can output IMU sensor data between 1 and 1,000 Hz, this will be the majority of the information downlinked from the CubeSat. The IMU outputs data at a rate between 9,600 bits per second and 921,600 bits per second depending on the sampling rate. At the minimum data output rate, the IMU generates about 829 Megabits per day. If only the IMU data and no other health data is transmitted, this is still significantly more data than the CubeSat is able to transmit. In reality the system would have other important data to transmit as well. This means the system will need to either have an ability to compress data or digitally downsample the data so there is less data to transmit as a whole. Typically to achieve lossless compression the rate can be no more than 
3:1, semi-lossless compression can achieve up to a 20:1 compression rate. If the IMU output rate is set at $1 \mathrm{~Hz}$ and all collected data is compressed to 20:1 ratio the data would still need to be dwonsampled at a 7:1 ratio. This is too sparse to effectively analyze the thruster firing. If 30 seconds per orbit that will capture the 20 seconds of thruster firing are compressed at the lossless rate of 3:1 the rest of the data can be compressed to 20:1 and downsampled at 8:1 to allow all data to be downlinked. Realistically higher sampling rate than $1 \mathrm{~Hz}$ will be desirable during the thruster firing, if the sampling rate is set to $20 \mathrm{~Hz}$ for the 20 seconds and the rest of the orbit at $1 \mathrm{~Hz}$ all data is compressed at the semi lossless rate of 20:1 and everything but the thruster firing is downsampled at a 20:1 all data collected in a day can be transmitted. The large increase in sampling during the firing would still result in more data to analyze even with the semi loss that occurs at a 20:1 compression rate. These are quick examples of the means that would allow the downlink budget to close, and inspection of the data actually exported from the IMU will likely lead to additional means to close this budget.

\subsection{Dynamics Model}

A significant part of this particular mission is a dynamics model of the thrusters firing. The current vision is to utilize this model to discern the real world effectiveness of the thrusters in orbit. This is accomplished by taking the recorded IMU data from an orbital testing, and comparing it to the expected values calculated by this model. This information is calculated in the body/IMU reference frame as this is the frame that the IMU data will be recorded in during flight testing. The $\mathrm{X}$ axis in this reference frame is parallel with the Sun, the $\mathrm{Y}$ axis is aligned with the direction of travel, and the $\mathrm{Z}$ axis is the resultant between the two. By knowing the mass and inertia tensor of the CubeSat in correlation with a known firing time the end rotational rates on all three 
axis can be calculated. By comparing the rotational rate of the three major axis to a multitude of scenarios dictated by different variables, the actual force of the thrusters can be determined. These variables include parameters such as bulk position tolerance of the thruster position, meaning the mounted position of the thruster differs from the modeled position using all or a portion of the dictated assembly tolerance. Another key variable is angular tolerancing of the thrust vector, which is the thrust vector being at some angle that is not orthogonal to the CubeSats reference system. Finally, the actual force of the thruster needs to vary in order to ensure that a model for any circumstance is achievable. To cover this array of scenarios a base dynamics model is developed. This base model can accept a three by three inertia tensor, and varies the angular alignment of the force vector. An inertia tensor represents the inertia of the craft about a given reference system. When the inertia tensor has no cross products the inertia tensor is representing the rotation about the principle axis of a system, every object has a reference system in which it has three principle axis. In reality to control the craft we need to define our own reference system which is based on the geometry of the craft. If the inertia tensor is measured in this defined reference frame and has cross products it means the principal axis are not aligned with the geometric axis of the system. To mitigate the effects of this frame rotation on the craft it is common practice to utilize a spin balancer to calculate how to effectively balance the craft and align these two reference systems. Equation 3.9 represents a system that has been effectively spin balanced and the principal and geometric reference frames are now aligned. Equation 3.10 has cross products and thus represents a system that has some frame rotation between its principle and geometric frames.

$$
I_{\text {balanced }}=\left[\begin{array}{ccc}
0.02788081 & 0 & 0 \\
0 & 0.006173500 & 0 \\
0 & 0 & 0.02736051
\end{array}\right]
$$




$$
I_{\text {unbalanced }}=\left[\begin{array}{ccc}
0.02778081 & -0.00054421 & -0.00007425 \\
-0.00054421 & 0.00617150 & -0.00020871 \\
-0.00007425 & -0.00020871 & 0.02716051
\end{array}\right]
$$

To ease the conveyance of information a system with its principle and geometric frames aligned will be referred to as balanced, while a system that has some rotation between these frames will be referred to as unbalanced. The magnitude of the cross products in an unbalanced system will increase as the system becomes more unbalanced, which means a torque in one axis increasingly effects the craft in the other two axes.

A large factor in this analysis is ensuring that it is similar to any future mission. A large aspect of this is the inertia tensors being used. To ensure the tensor was comparable with a future flight mission, the CubeSat is approximately created in SolidWorks. This model includes the correct masses and volume for all components, and the placement was chosen to naturally balance the craft as much as possible. The design of acceptable support structure was not completed in order to minimize the overall scope of the thesis. This effort results in the above unbalanced inertia tensor shown in Equation 3.10. Additional effort into balancing the inertia tensor manually resulted in a more balanced tensor, with much smaller cross products. The cross products on this tensor are set to zero, to help in understanding the effect the cross products make on the end rotational rates. This model is represented the balanced inertia tensor Equation 3.9.

Initially, the force vector is orthogonal to the reference frame of the CubeSat which in this case means it is perfectly parallel with the $\mathrm{Z}$ axis. The vector is then rotated one degree off of perfectly orthogonal to now be comprised of $\mathrm{Z}$ and Y components. This new vector is then rotated about the original $\mathrm{Z}$ axis 360 degrees, causing the 
vector to be comprised of $\mathrm{Z}, \mathrm{Y}$, and/or X components. At each step the end rotational rates are calculated. The thruster should realistically be able to be mounted within 10 degrees of orthogonal, thus the initial rotation of the force vector is repeated 5 times for a total angular misalignment in one direction of 5 degrees. This allows the total misalignment tolerance to be run in a single model and display the overall effect of misalignment. As the vector components vary they interact with lever arms of variable lengths and the inertia tensor to vary the angular rates in the three major axes. Figure 3.22 shows the relationship between different components of the force vector and the lever arms within the craft with their effect on the overall torques experienced by the craft. The above described method of calculating is done for both the thrusters and has the utility to vary to what degree the forces couple or cancel as they are calculated independently.

The calculated torques acting on the craft are assembled into a torque matrix, T, which includes the torque about the $\mathrm{X}, \mathrm{Y}$, and $\mathrm{Z}$ axes. This torque, the above inertia tensors, I, and the current rotational speeds, $\omega$ are utilized in Euler's rigid body dynamics differential equation solving for the rotational acceleration, $\dot{\omega}$, which is shown in Equation 3.11. This equation is solved inside a custom function running within ODE45 in Matlab. The ODE function integrates the rotational acceleration calculated in the custom function and outputs the rotational rates in all three axis. These analyses all use a firing time of 20 seconds, the flight mission firing time may vary but determining an accurate firing time in orbit will be possible using the gyroscope data during the performance analysis.

$$
T=I \times \dot{\omega}+\omega \times(I \times \omega)
$$

To begin, the bulk position of the thrusters is assumed to be as modeled, meaning the 

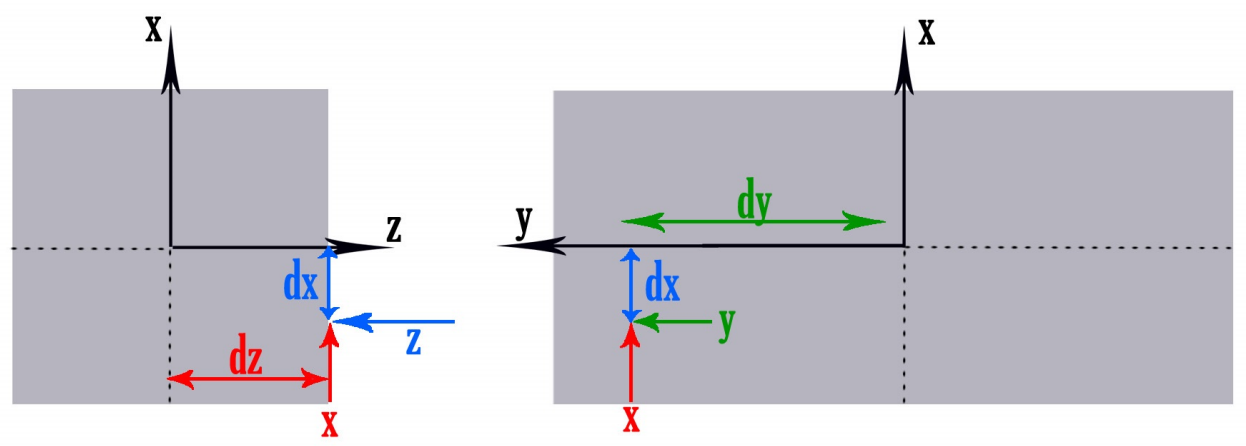

$$
\begin{aligned}
& T x=\left(\begin{array}{ll}
z & d y
\end{array}\right)+\left(\begin{array}{ll}
y & d z
\end{array}\right) \\
& T y=\left(\begin{array}{ll}
x & d z
\end{array}\right)-\left(\begin{array}{ll}
z & d x
\end{array}\right) \\
& T z=\left(\begin{array}{ll}
-x & d y
\end{array}\right)-\left(\begin{array}{ll}
y & d x
\end{array}\right)
\end{aligned}
$$

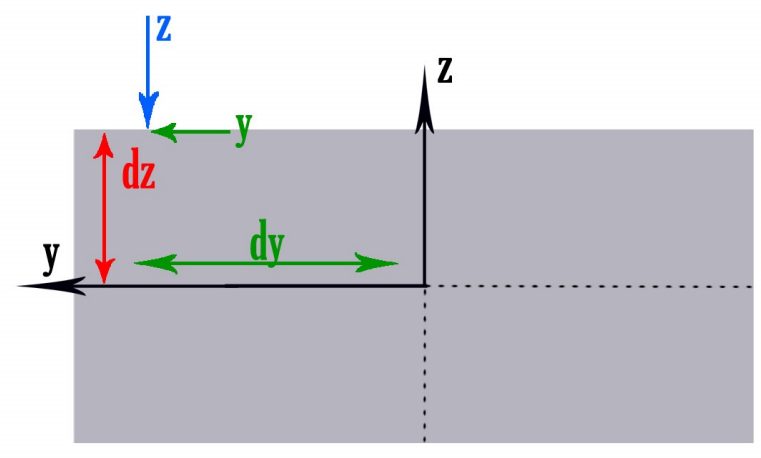

\section{Figure 3.22: Components of the Thrust Force and Various Lever Arms Within the Craft and Their Relation to the End Rotational Rates of the craft}

thruster is placed perfectly within the craft. When the thrusters are placed in this manner, the inertia tensor is balanced, and the thrust vector is orthogonal to the craft, there is only rotation about the intended axis as shown in Figure 3.23. When the above described method of angling the vector is correlated with variable force of the thruster three dimensional plots are created. These plots have the variable power in one axis, the degree of rotation about the $\mathrm{Z}$ axis described above as another axis, and finally the end rotational rate in the final axis. When plotted in this manner multiple iterations are graphed to encapsulate the overall alignment tolerance, and its effect. The large magnitude difference between end rotational rates about the intended axis compared with the unintended axis makes the effects on the unintended axis difficult to visualize. So, one plot contains the rotational rates for the five degrees off of 
orthogonal for both the $\mathrm{Y}$ and $\mathrm{Z}$ axis seen in Figure 3.24 while a separate plot shows the effects of this angle change on the intended $\mathrm{X}$ axis seen in Figure 3.25.

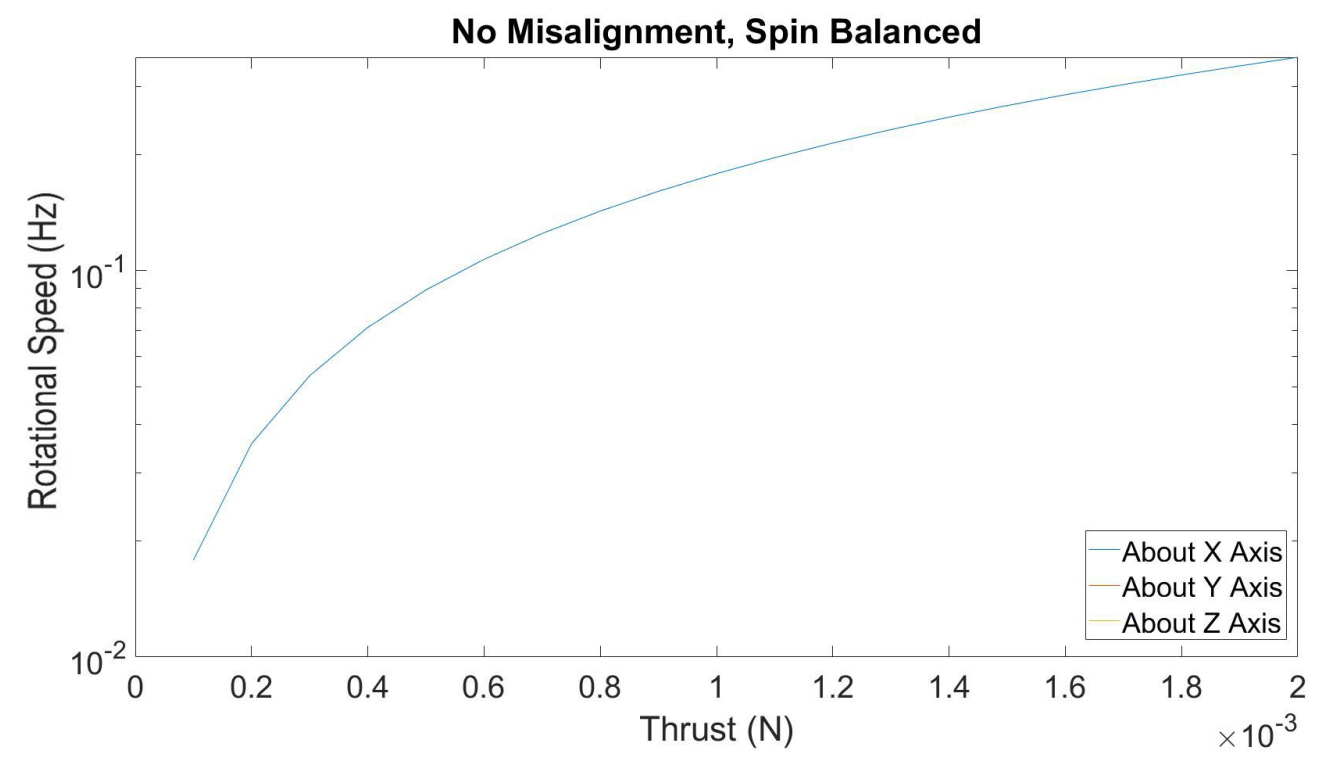

Figure 3.23: Resultant Tumble When Thruster Is Perfectly Aligned and Inertia Tensor is Balanced

Unintended axis, Spin Balanced

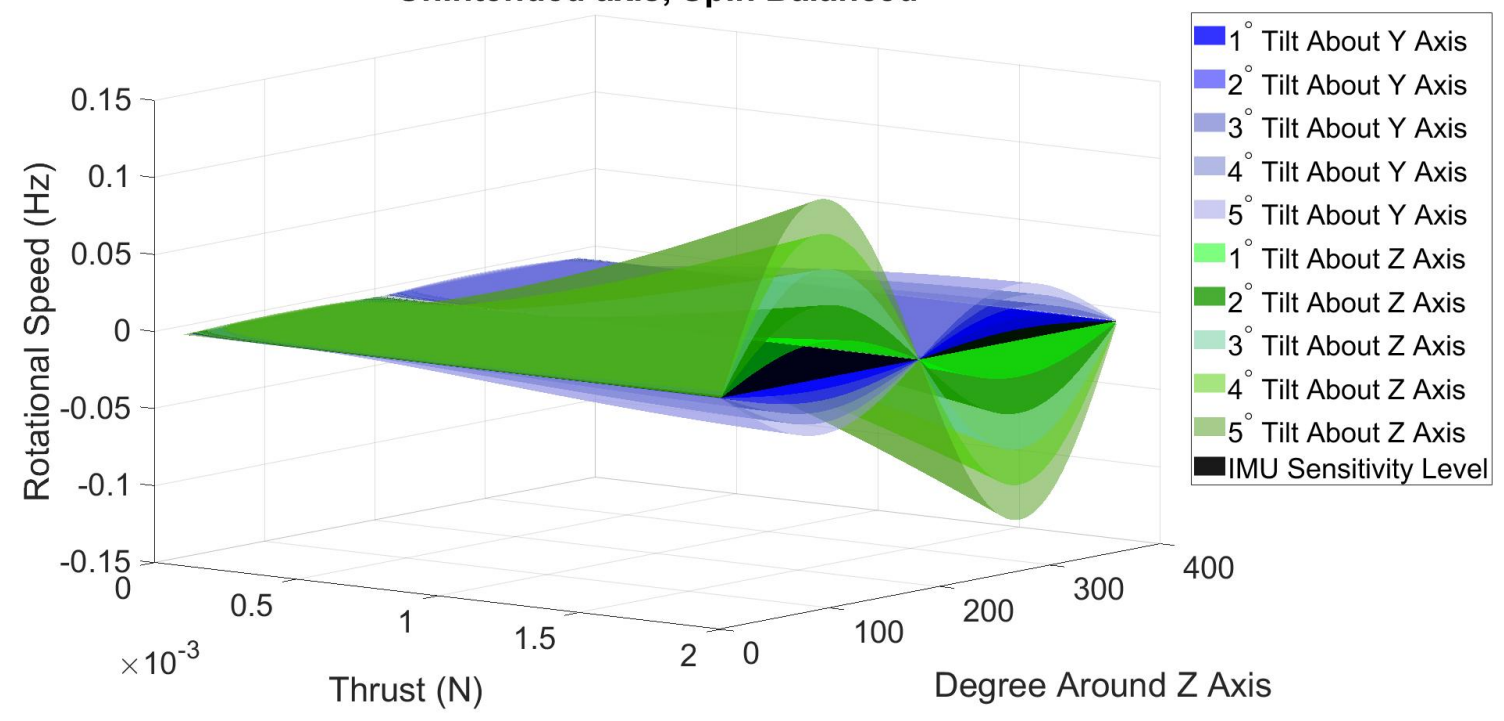

Figure 3.24: Resultant Tumble on Unintended Axis as the Thrust Angle Changes and Inertia Tensor is Balanced

Figure 3.25 show the intended axis rotational rates are much higher than the rotational rates in the unintended axis Figure 3.24 as is expected. These initial plots 


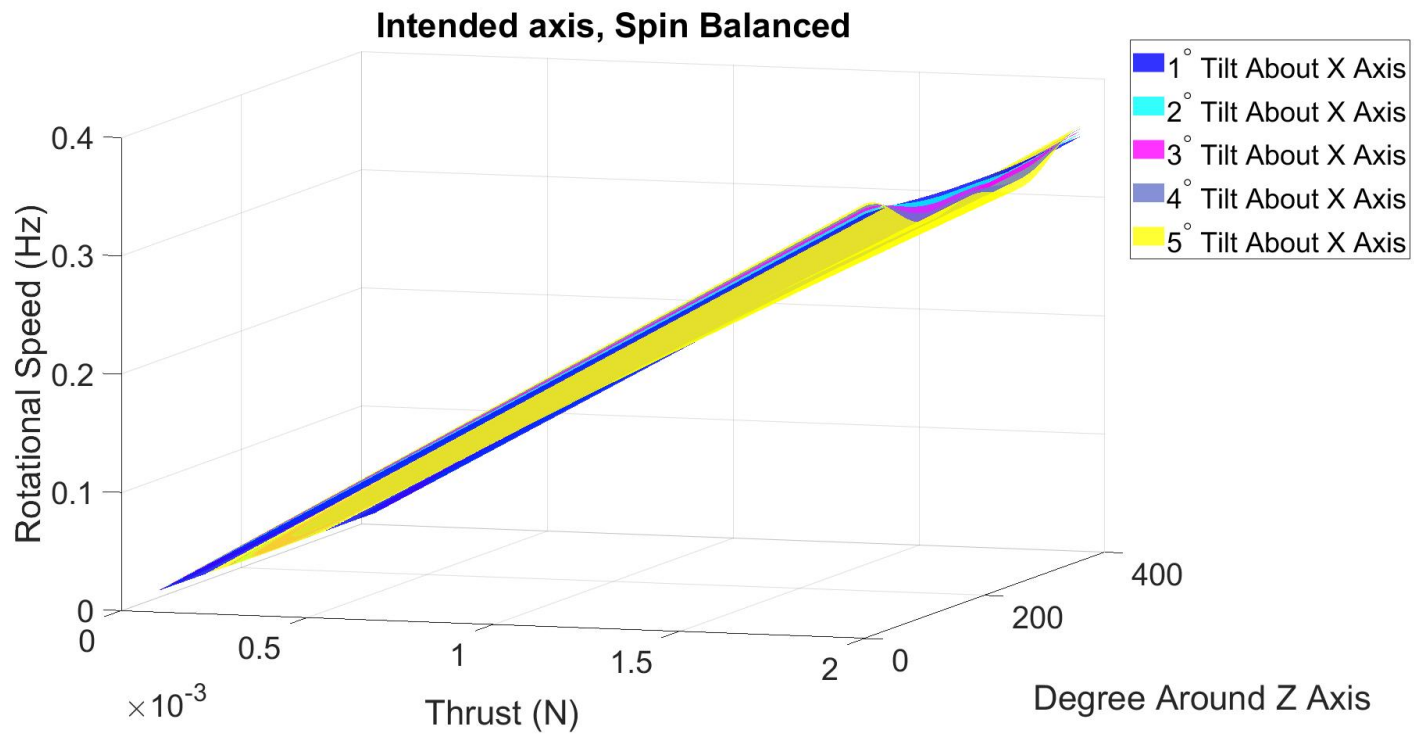

Figure 3.25: Resultant Tumble on Intended Axis as the Thrust Angle Changes and Inertia Tensor is Balanced

represent a balanced inertia tensor, this is accomplished by spin balancing a physical system to eliminate the cross products in the inertia tensor. Spin balancing wouldn't be able to completely remove the cross products of the inertia tensor, but bring them to extremely low levels to minimize their impact on the system. Spin balancing is the standard operation for flight systems utilizing ADCS. This is due to the nature of a torque in one axis acting on an unbalanced inertia tensor resolving in a complex tumble, i.e. one that has rotational rates about more than one axis. Depending on the funding level available for this mission spin balancing may not be an option so considering an unbalanced tensor and comparing its effect on the system is necessary.

As seen above, the unbalanced inertia tensor results in rates in all three axes even in the orthogonal case shown in Figure 3.26. The plots representing the misalignment of the thrust vector rates show significant difference in the end rates compared to the balanced system. The tensor used in Figures 3.23, 3.27, and 3.28 is reflective of an effort to manually balance the inertia tensor. As the inertia tensor becomes more unbalanced the effects become more drastic. The following plots represent a worse 


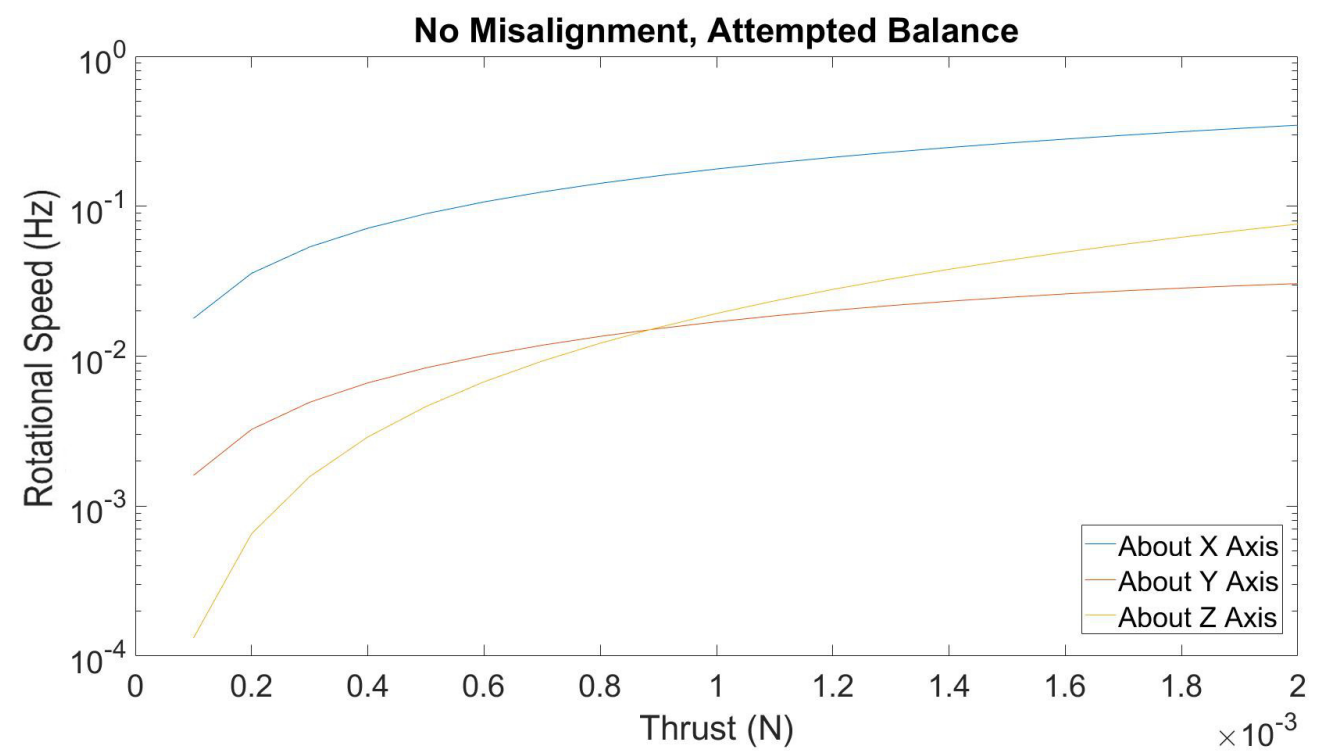

Figure 3.26: Resultant Tumble When Thruster Is Perfectly Aligned and Inertia Tensor is Unbalanced

\section{Unintended axis, Attempted Balance}

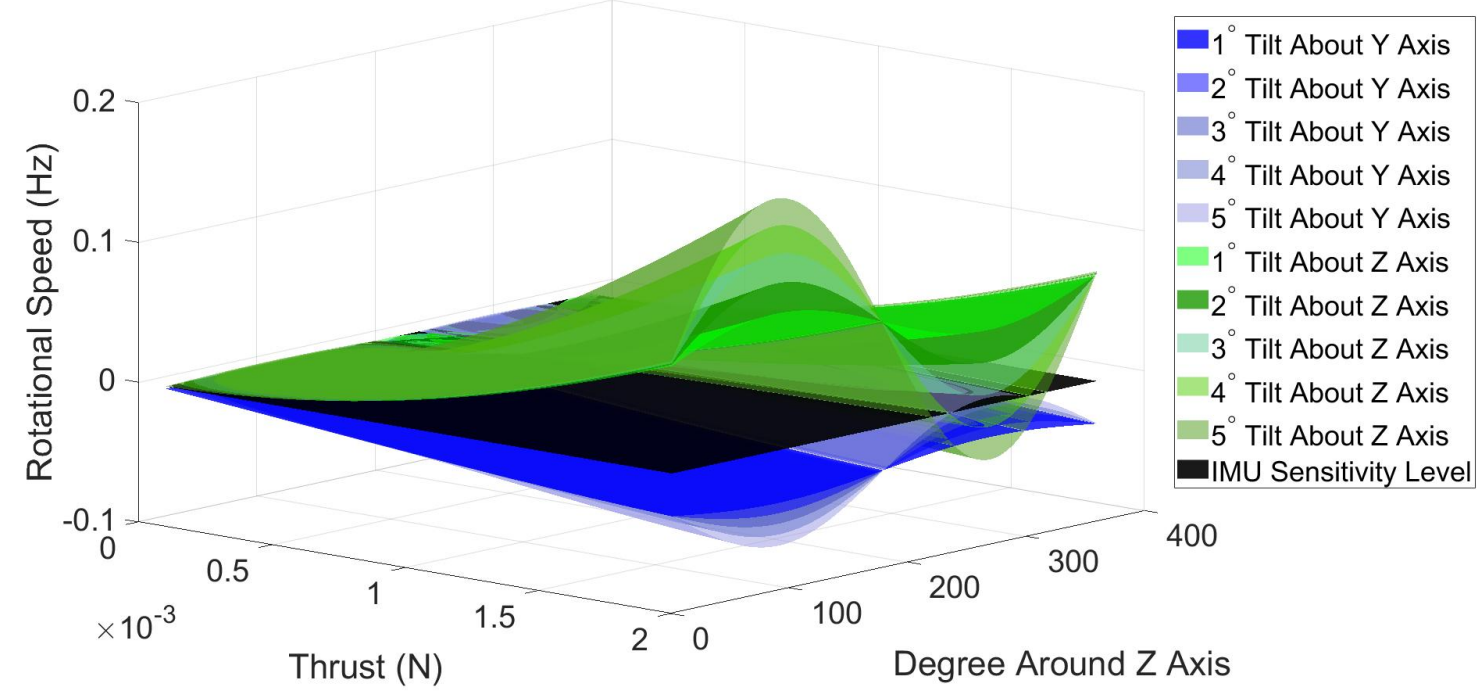

Figure 3.27: Resultant Tumble on Unintended Axis as the Thrust Angle Changes and Inertia Tensor is Unbalanced

case scenario in which the additional un-modeled mass, such as wires or screws, is biased in a single direction severely unbalancing the craft. The assumption that the inertia tensor is constant is also made, as the thrusters fire mass of argon gas is lost at either end of the craft. The total mass of argon onboard the craft is .0036 grams, 


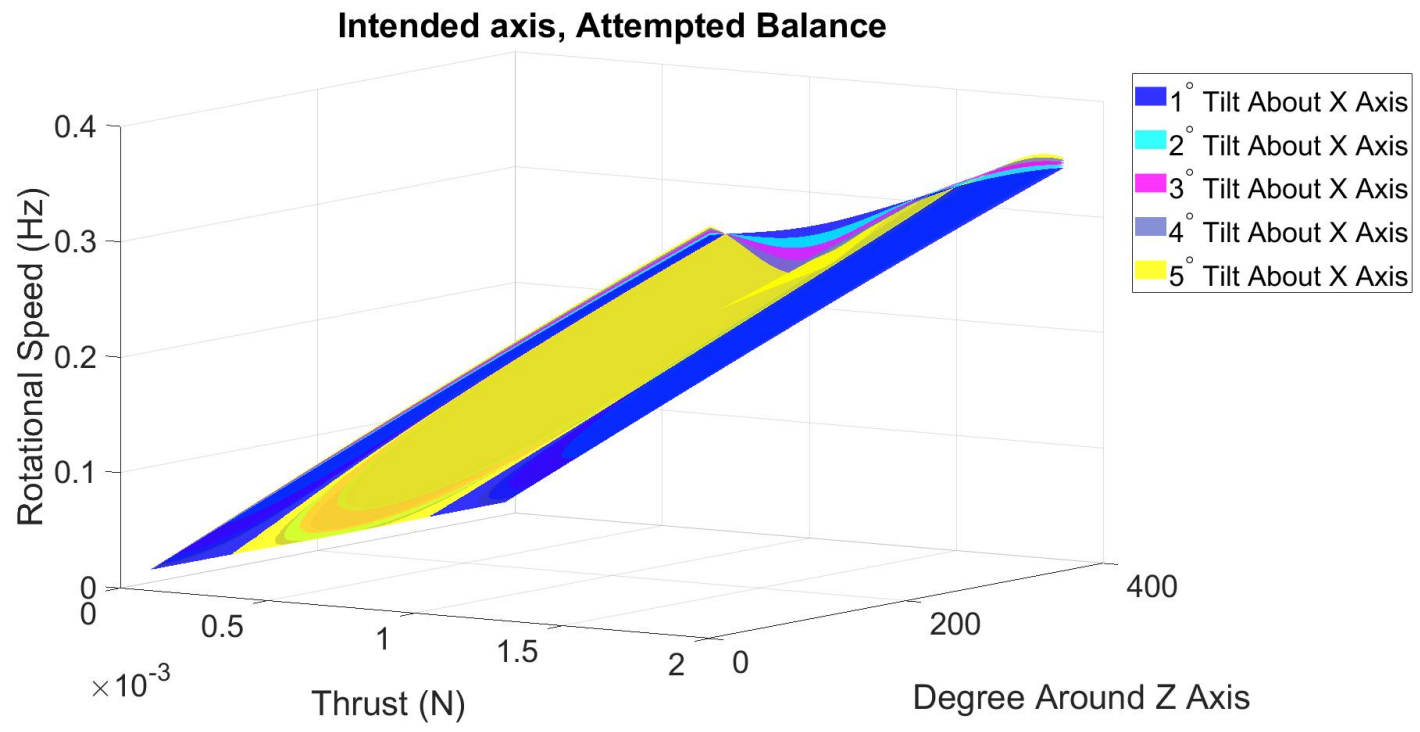

Figure 3.28: Resultant Tumble on Intended Axis as the Thrust Angle Changes and Inertia Tensor is Unbalanced

as this is 0.00012

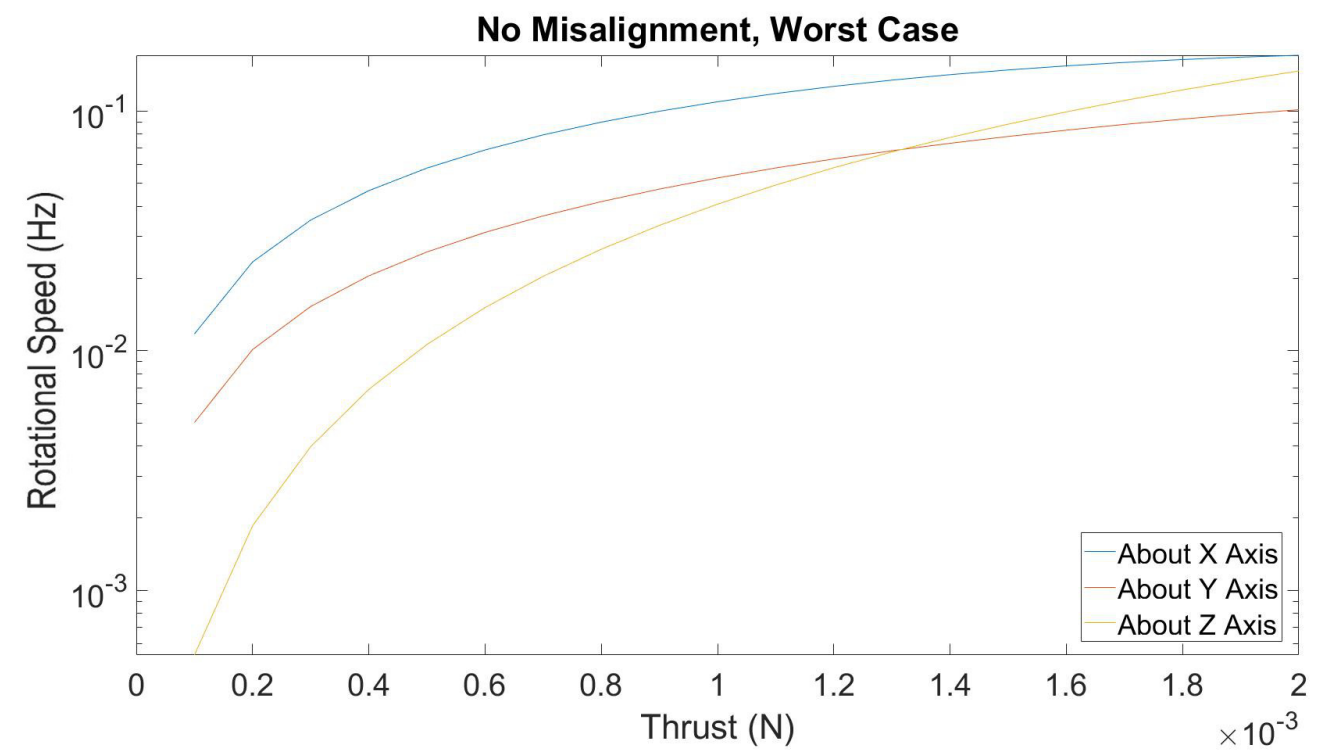

Figure 3.29: Resultant Tumble When Thruster Is Perfectly Aligned and Worst Case Inertia Tensor

As is apparent in Figure 3.32, this worst case scenario inertia tensor results in more severe complex tumbles as the thruster is fired. Once the craft is unbalanced the effect on the intended axis will be minimal as the craft becomes increasingly unstable. The 


\section{Unintended axis, Worst Case}

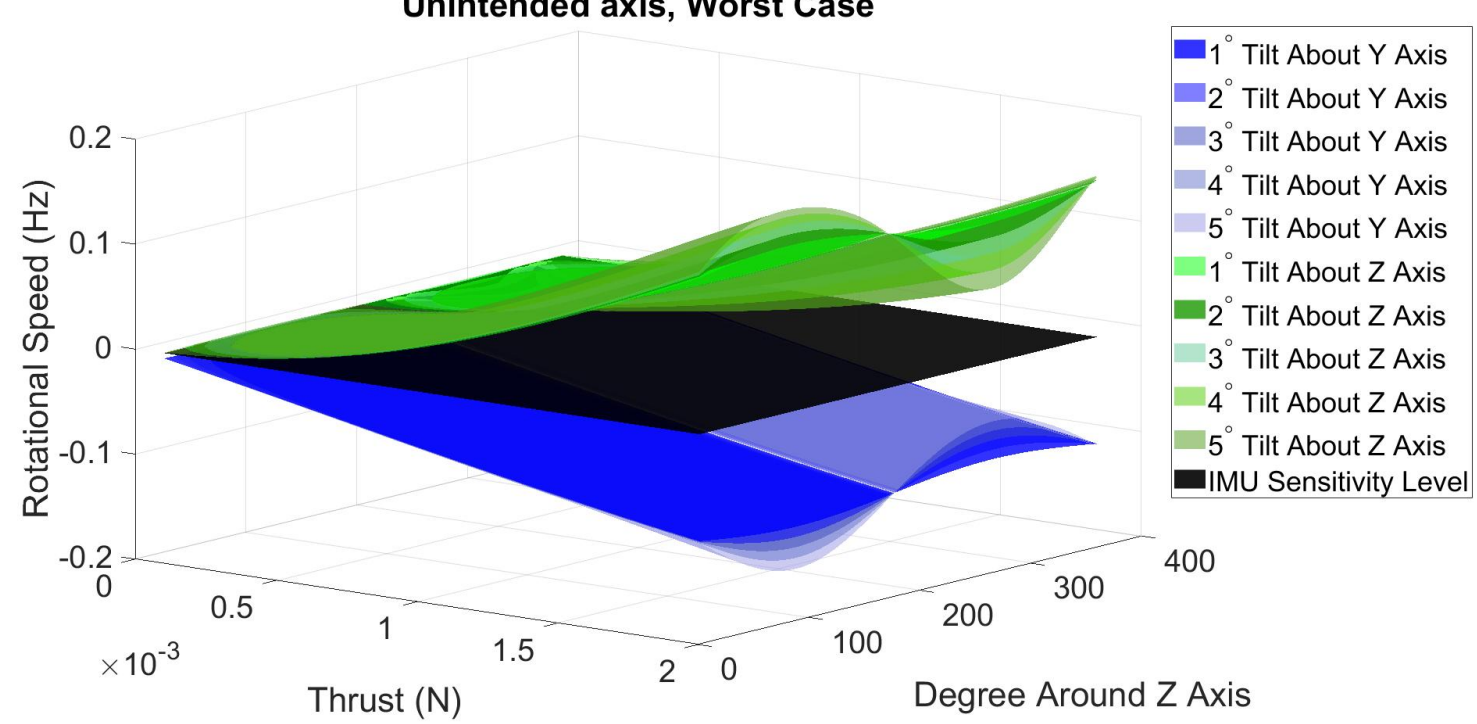

Figure 3.30: Resultant Tumble on Unintended Axis as the Thrust Angle Changes and Worst Case Inertia Tensor

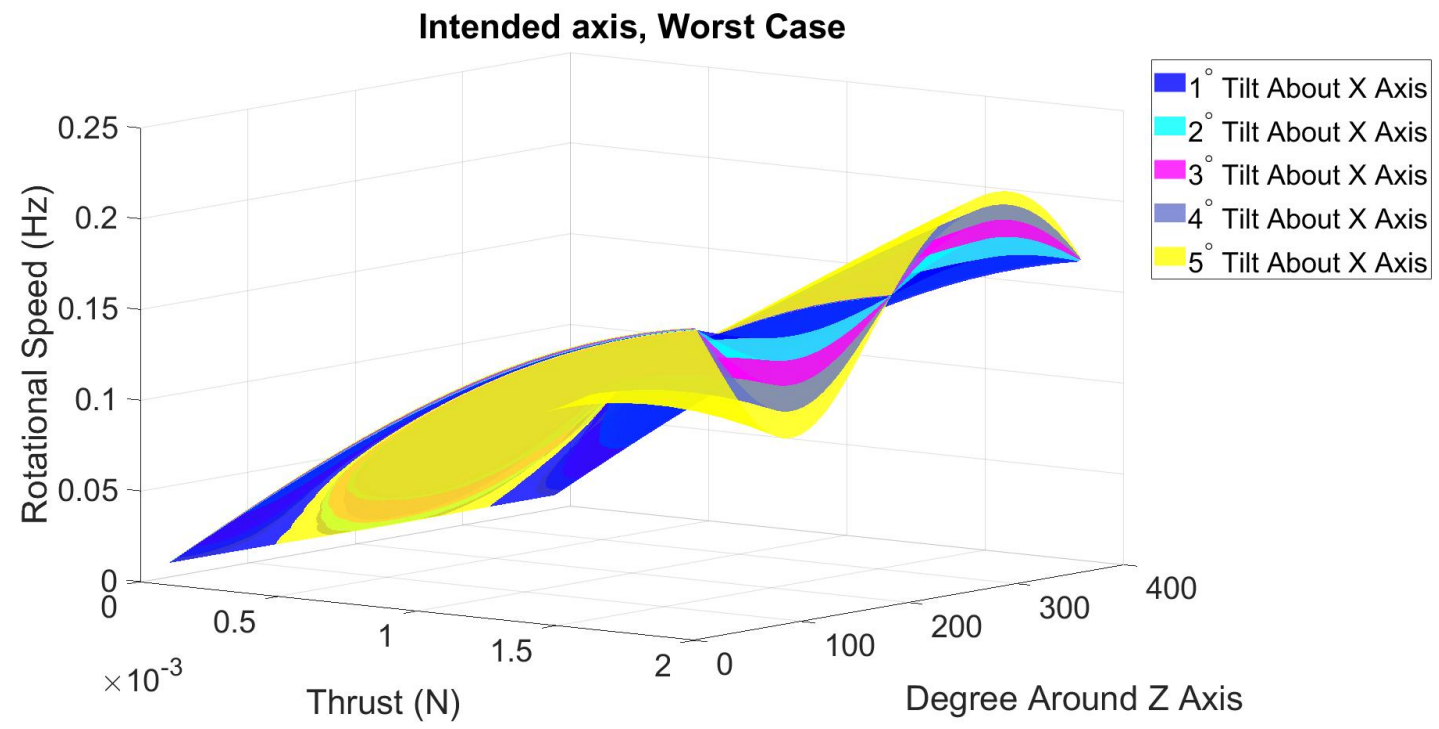

Figure 3.31: Resultant Tumble on Intended Axis as the Thrust Angle Changes and Worst Case Inertia Tensor

magnitude of the end rotational rate along the unintended axes will increase in both or individually depending on the inertia tensor itself. If these rates become too great it could result in an unrecoverable tumble. Similar effects would occur every time the reaction wheels attempt to stabilize the craft, to counteract this the reaction 


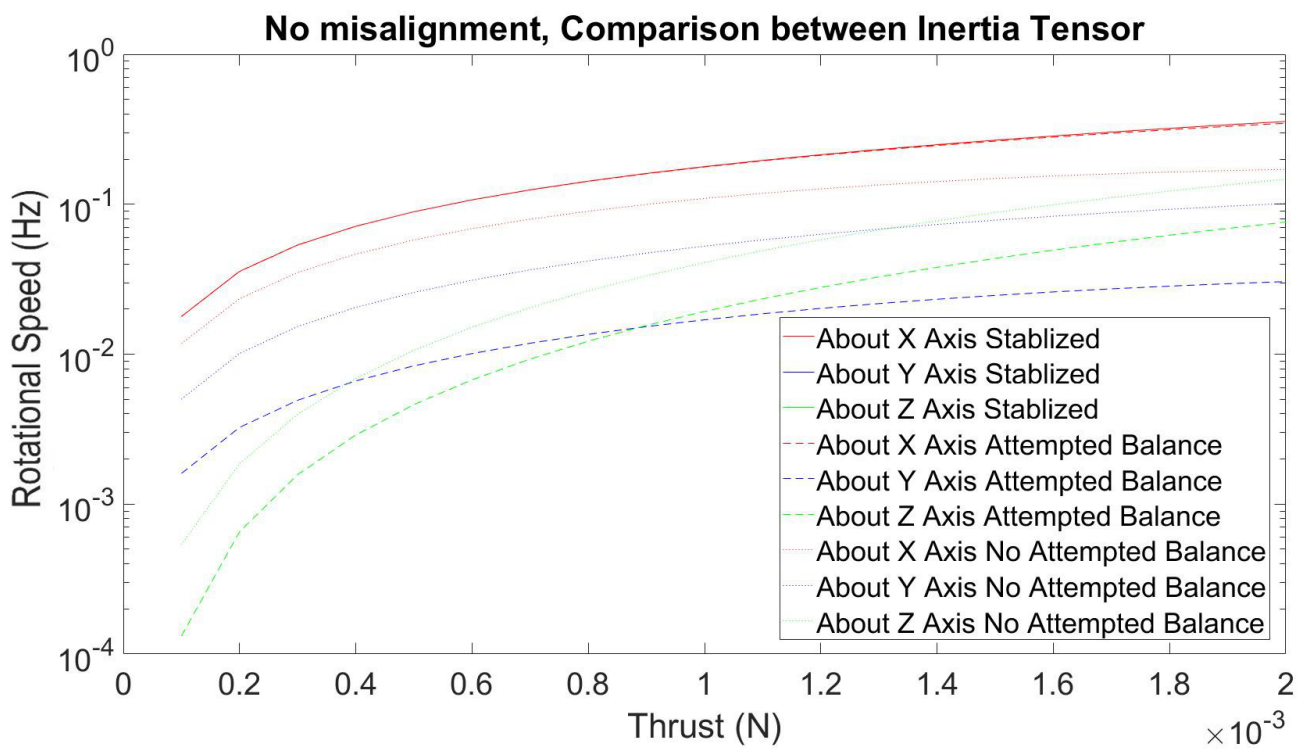

Figure 3.32: No Misalignment of Thruster Compared Between Different Inertia Tensors

wheels would have to be programmed to accommodate this effect which would be significantly more complex. The difficulty in using an inertia tensor calculated by a computer aided design program, is that the mechanical design often does not include things like cable routing, tie downs, or the realistic tolerance of an assembled system. This would mean that the actual tensor is unknown. Based on this data, at a minimum measuring the inertia tensor of the flight craft is required to be able to accurately control the system. Balancing the system would simplify the control of the craft, and increase the repeatability of the thruster firing experiment while minimizing the risk of the system entering an unrecoverable tumble.

The angle of the thruster is not the only variable used within the dynamics model, another key factor alluded to in the above description is the bulk placement of the thrusters. The model has the ability to shift the location of the thruster thus effecting the length of the various lever arms shown in Figure 3.22. Based on standard machine tolerancing, it is very feasible to place the thruster within a 0.254 millimeter true position, meaning the thruster can shift anywhere in a radius of 0.127 millimeter and 
be within acceptable tolerance. This results in a few interesting cases. For example if the thrusters move away from each other in a direction parallel to the $1 \mathrm{U}$ face. This results in coupled torques creating the highest "roll" rate on the spacecraft as well as the intended tumble.

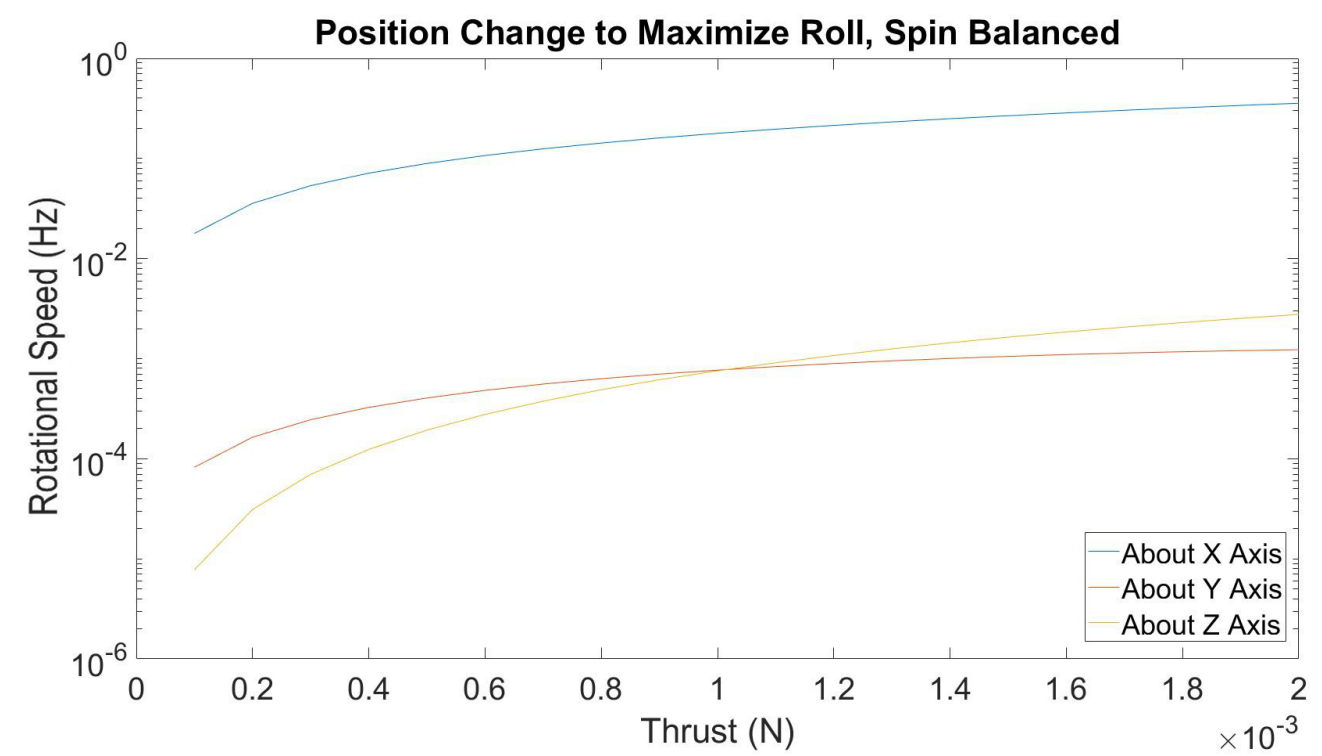

Figure 3.33: Thruster Repositioned to Maximize Roll, With a Balanced Inertia Tensor

Figure 3.33 represents a balanced inertia tensor and the thruster offset in such a way to result in an attempt to roll while still being in the positional tolerance discussed above. As you can see, the effect is a tumble through all three primary axes. The smaller rates about the $\mathrm{Y}$ axis is caused by the slight length of the lever arm that forces roll. This new lever arm is a fraction of the length of the lever arm intended for the thrusters. This is one of the reasons that a $3 \mathrm{U}$ craft is more effective than smaller systems. The increased lever arm for the thruster results in a more dominate tumble in the intended axis. The tumble about the $\mathrm{Z}$ axis is caused by the relationship between force components in the torque equation detailed above in Figure 3.22, and the inability of the craft to tumble on only two axes.

The overall intention of this models flight operation is to measure, as accurately as 
possible, different variables of the flight system before launch. By having accurate position of the thruster values, alignment of the thruster angle, and the inertia tensor in hand the overall accuracy of the analysis can be increased. The goal is to minimize the breadth of the analysis to minimize the risk of having two distinct scenarios with potentially different functionality match the results measured in orbit.

A key aspect to this is the IMU actually being sensitive and accurate enough to measure these sometimes small rates. The selected IMU measures the rotational rate for all three major axis to $8 \times 10^{-} 6$ Hertz and as a result is capable of measuring the vast majority of scenarios. There are some very brief angles where the rate about a given axis switches from positive to negative where the resultant rates are below the threshold, however, this is uncommon. This means almost all scenarios of measured flight data would result in accurately identifying a scenario within this model that matches the real world experiment.

It is also necessary to consider the disturbance torques associated with an orbit at this altitude. These are namely aerodynamic drag and solar radiation pressure. To simplify the analysis, it assumed the aerodynamic flow and the solar vector are parallel to an axial plane of the craft and thus only acting on two faces of the craft at any one time. In this case one face is 10 centimeter by 30 centimeter while the other face is 10 centimeter by 10 centimeter. Any orthogonal force component will not impose a torque on the craft as there would be no lever arm to act against. This means only the force components parallel to one of these two surfaces impose a torque. The drag coefficient for an angled plate is being used to represent the face of the craft at an angle to the flow. The atmospheric density at $500 \mathrm{~km}$ is extremely low, this results in extremely small torques. Applied for the same 20 second time scale as the science objective, to allow for direct comparison, the resultant rotational rate is in the $10^{-4}$ order. The solar pressure is equal to the solar irradiance divided by the speed of light. 
This pressure multiplied by the surface area of a face results in a force acting on the craft. When applying this torque for the same 20 second time period, the resultant rates are again in the $10^{-5}$. Figure 3.34 shows the rotational rates caused by these disturbance torques acting for a period of 20 seconds.

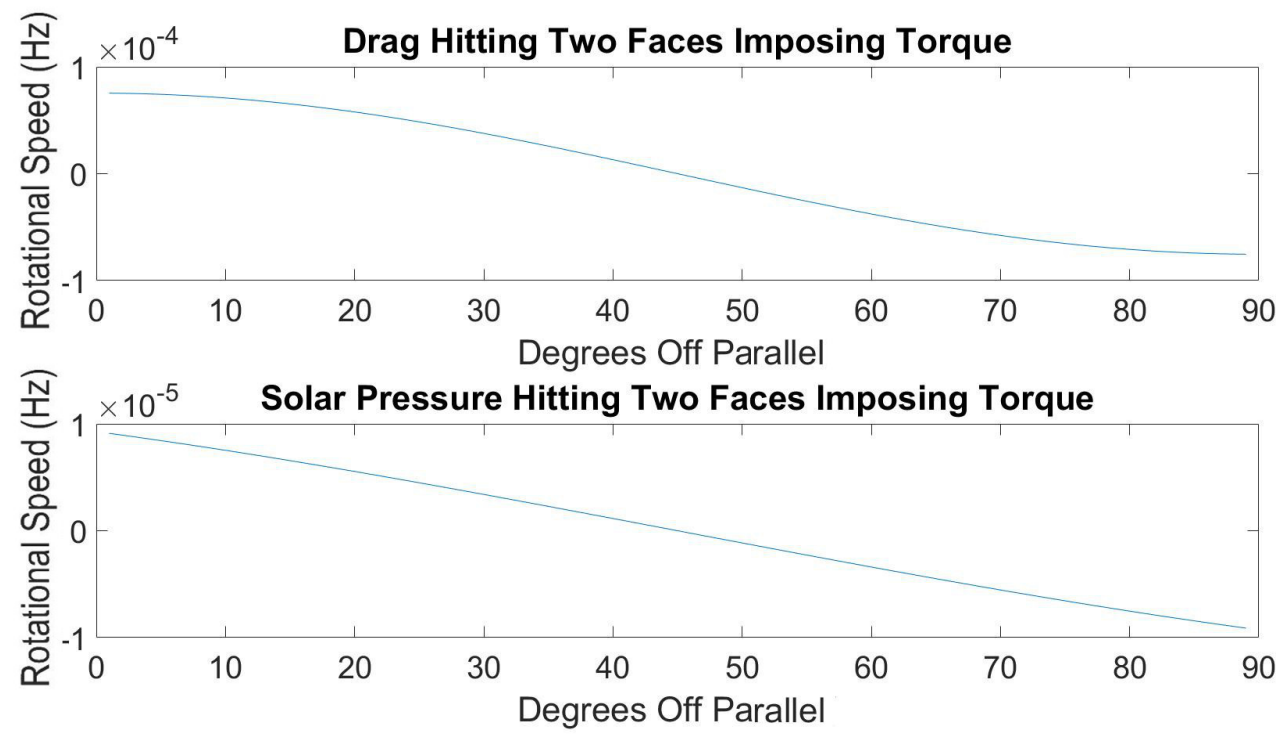

Figure 3.34: Plot of Disturbance Torques Effects as Incident Angle Varies

The resultant tumble rates are lower than the expected rates in all three axes by 1 to 3 orders of magnitude for the majority of examples above. The only scenario in which rates this low are found is a perfectly orthogonal thruster firing below expected thrust acting on an imbalanced system, where the resultant rotation about an unintended axis is in the $10^{-4}$ order. These disturbances are large enough to be seen by the IMU, however are so small they would not realistically change the calculated functionality of the craft. It is worth mentioning, that the aerodynamic drag at this height is in the rarefied gas realm and would not be a consistent force in the system. The Sun pointing control law should keep a single face square to the Sun, which would result in no torque to the craft. After desaturating the reaction wheels, the craft will enter an inertial tumble, which opens the possibility of the solar vector incidence angle varying from zero. At this point, or any point during a complex tumble, the solar radiation 
pressure has the ability to impose a torque to the craft thus resulting in some tumble.

This analysis highlights the need to regulate the firing time for the thrusters. In the scenarios in which pocket rocket generates twice the thrust as expected, the resultant angular momentum is too great for the selected reaction wheel bundle to stabilize alone. This is still a recoverable scenario by utilizing both the reaction wheels and magnetorquer in tandem, but this is non-ideal. As it will take longer and potentially risk depleting the stored power before the craft resumes generating power. If Pocket Rocket is found to be over performing shortening the length of time the thruster is firing will eliminate this risk.

The analysis highlights the unique response the system can expect to a given combination of variables, this reinforces the idea that the end dynamic response can be traced to a true thruster functionality. Further, it highlights that knowing the inertia tensor of the flight CubeSat is paramount to determining the true thruster functionality. Additional analysis also showed that the location of the center of gravity (CG) does not need small tolerances, the torque balances well due to the linear relationship between coupled torques. The larger effect is the unbalanced mass caused by an uncentered CG effecting the inertia tensor. This is surmountable by effective ballasting, the worst case inertia tensor's CG is 2.54 centimeter off of the geometric center of the craft and although this would be an extremely difficult craft to control. The response is predictable with the proper inertial tensor, aiming for a center of gravity within a 0.635 centimeter $4 \pi$ steradian tolerance zone would result in a much more controllable CubeSat. However, the real tolerance should be applied to the inertia tensor, and that will vary with the specifics of the CubeSat. 
Chapter 4

\section{FUTURE WORK}

A significant amount of work is still necessary before this proposed CubeSat mission is ready for launch. A notional path forward is outlined in this section, as well as additional work that could aid in the mission feasibility. This additional work is proposed for student investigation and consideration.

\subsection{Identifying Potential Future Student Work}

There are several endeavors that could be undertaken by future students that would aid in making this mission even more feasible for the Cal Poly CubeSat Lab (CPCL). As identified in the dynamics section, having a spin balanced system makes the operation of an attitude determination and control system significantly more straightforward. This is due to the system's inertial tensor having the smallest cross products achievable as well as known cross products, resulting in predictable and repeatedly dynamic response. This is accomplished through the use of a spin balancer which can be extremely expensive, however, the development of a cost effective system that would perform this task should be within the means of the school. This thesis and an upcoming CPCL mission, ExoCube 2, used an inertia tensor calculated by a computer aided design system as the flight tensor that would be utilized by the ADCS. This requires an incredibly in depth modeling effort to accurately calculate what the flight systems inertial tensor will be, and will still likely differ to some extent due to manufacturing tolerances. It is significantly more effective to know the actual inertial tensor of a final flight system and use that input for calculations by the ADCS on 
board the CubeSat. A second component would be a software that takes the size and weight of the system, as well as the measured inertial tensor to calculate an effective ballasting strategy to cancel the inertial cross products. This would need to limit the shimming to the outside surfaces of the craft to ensure the ballasting is possible without significant additional mounting, as this would only further change the inertial tensor. This would likely need significant time and commitment to achieve, and both seem a worthy endeavor for a Masters level thesis. One to determine a reasonable means to measure the inertia tensor, another to create a software that will calculate how to ballast the system to eliminate the cross products.

The reaction wheel bundle is a significant cost to the mission, therefore development of a reaction wheel system for the use by CPCL would enable more mission variety while keeping flight costs low. Reaction wheels are not mechanically complicated, but their difficulty lies in effective repeatability of the components within. The fly wheel portion needs to have a predictable and balanced weight to work effectively and not induce extra vibration to the craft. Additionally, any mounting also needs to be effectively toleranced to get the expected effect from spinning. If the above mentioned spin balance or inertial tensor measurement system comes to fruition, they would be extremely useful for quality control for the fly wheel component of the individual reaction wheels. The inertial tensor measuring system could also accurately measure the applied torques of a reaction wheel bundle in correlation to a power draw. This would be accomplished by spinning a bundle and activating wheels to measure the inertial tensor change in relation to the bundles speed. This would be an extremely helpful tool in developing the necessary control to gain a desired effect. Which segues in to the other important aspect that would need to be addressed, the control software for the wheels. The ability to read the IMU data and initiate a specific amount of movement with one or more wheels to balance the effect is complicated and will need to be developed to run with CPCL hardware. This control software will require a 
significant effort if it is necessary to design from scratch, even a framework meant for a different system could potentially require a significant overhaul to be adapted to the CPCL hardware. This would need to correlate with lab testing to determine the effect of a physical bundle to ensure the accuracy of this control software.

\subsection{Future Mission}

The future mission would require significant additional work beyond this thesis. As the goal was to analyze the feasibility, this thesis merely laid the foundation. The first major goal will be securing the funding to allow the mission to progress. To aid in the explanation of necessary future work, the mission has been divided into two main disciplines mechanical and electrical design. In this case, the software development necessary is being considered under the electrical discipline. A major electrical undertaking will be the circuitry redesign of the CPCL flight computer to allow the use of the reaction wheel bundle, IMU, and magnetorquer board. This will require a minimum of adding connectors, if not needing additional processing to handle the new and different functions the board will require. Software will also need to be added to the board to help in communication between the flight computer and the given hardware. An additional issue would be managing inertial drift, over time error stacking will make the IMU read angular rates that differ from the actual values the craft sees. The flight computer will need to be able to correct this misinformation. This thesis makes the assumption that the on board magnetometer data will be enough to correct this inertial drift. However, this will need a closer look in the future to determine if additional hardware is necessary, additionally the software to actually take this information and recognize the drift and then correct it will need to be developed. Another major software endeavor is the actual development of the control law that governs the satellite. This thesis uses a simple PID controller 
to enforce a Sun pointing law based on a pre-calculated Sun position, utilizing the Julian date and the position of the craft. The flight control law will likely need to be more complex, and allow the craft to enter an inertial tumble for the firing of the thrusters. It will then need to restabilize and resume its Sun pointing control. Finally, as detailed in the link budget section, the craft will require some compression software that can compress the large amount of data being generated by the IMU to be transmitted in a timely fashion. This may need to be extended to a delimiting functionality if the compression is not enough individually. The software development is likely a significant time commitment for the team and will be need to be undertaken by someone with more coding experience.

The mechanical side of the mission is largely the full mechanical design of the actual CubeSat. This thesis limited itself to a first order design, which included a rough layout of all the identified hardware. A full design will need to include a more detailed layout, with a structure that can accommodate the mounting of all hardware and be secure enough to last through expected vibrational environments. The full design layout for some CPCL hardware was not available for publication, this information may warrant another look into the thermal conditions within the craft. Heat sinks that provide a more direct path from the hot spots on one of these boards to a radiative surface may prove necessary. The thermal analysis detailed previously accounts for this energy being generated, so the overall craft temperatures should not change significantly. As previously mentioned, CPCL has experimented with deployable solar panels on the ground. Before their use in this mission more work to ensure a reliable deployment to a known position will need to be performed to minimize the effect on the inertial tensor as well as decrease the risk of the panel deployment failing.

Funding is likely the largest hurdle standing before the actual flight of this proposed mission. However, some of the work detailed in "Identifying Potential Future Student 
Work", Section 4.1, is general enough to seek independant funding to improve the effectiveness of the CPCL team. The inertial tensor measuring system, the software that allows for tensor balancing, a CPCL reaction wheel system, as well as its control law, and reliable deployable panels are all systems that could be undertaken as student projects with minimal associated cost. These systems would vastly increase the available mission types for California Polytechnic State University, including this mission. The development of a fully functional reaction wheel bundle, as well as the deployable panels would directly reduce the amount of work necessary to perform this mission while reducing the overall cost of the mission. This is due to tertiary effects, such as realistically already having the ability to run this newly developed reaction wheel bundle with a CPCL flight computer while also taking input from a pre-selected IMU. 
Chapter 5

CONCLUSION

The above analyses show the feasibility of using CubeSats as a low cost orbital test bed for propulsion systems. The methodology proposed for measuring the thruster performance is non typical for a spacecraft mission, and allows the testing of extremely small thrusts for a fraction of the cost of other measuring techniques for the cost of added complexity. While the solutions proposed to address other problems identified by this work are not as unique, they represent required new steps for the Cal Poly CubeSat Lab to successfully accomplish missions of this type. Many of these design decisions were made to minimize the complexity and cost of the craft, and as a result do not always represent the best or most efficient way to solve the problems identified. The large breadth of topics concerning the requirements and spacecraft design options described above, can all be investigated further to determine the true intricacies and sensitivities of designing a CubeSat orbital test platform. The design detailed above is the result of relatively light analysis compared to the level of analysis necessary to actually fly a spacecraft. However, these simplified analyses show that a mission of this type can fly successfully under reasonable assumption. This is merely the first step in the long road to creating and flying a satellite, however, they are important steps none the less.

The analysis on the power budget concludes that a Nadir pointing control law would be unable to generate enough power to support this mission, even in a scenario in which all surfaces are covered in solar panels. A Sun pointing control law requires the addition of deployable panels to increase the power generation, the addition of two $1 / 2 \mathrm{U}$ x $3 \mathrm{U}$ panels which effectively doubles the power generation. This allows 
the power budget to close, with margin, over a year long period.

The transient thermal model revealed the viability of an extremely SWaP effective passive heat management system. This system is comprised of a gray paint that has an absorptivity of 0.6 and an emissivity of 0.9 . It is worth noting that the UTJ Solar Panels have higher an absorptivity of 0.92 and an emissivity of 0.85 . As the face utilizing the UTJ panels is always sun pointing it is the largest source of heat for the system, its higher absorptivity and lower emissivity coupled with its reduced effectiveness at radiating away heat results in this face being hotter than the rest of the craft. This is caused by inefficient heat path to transfer heat away from the solar panels. This is solved by adding small aluminum thermal straps between the solar panel face and every other face of the craft. With this additional heat path, the faces are more in family and the overall temperature of the craft is lowered due to the ability to move heat to faces which are more effective at radiating thermal energy away. This solution requires no power to function and thus has no effect on the power analysis.

The communications analyses shows that the link budget will close with margin at a viewing angle of $15^{\circ}$ above horizon. This link closes without the need to point the antenna on the CubeSat, meaning that there is no need to vary the sun pointing control law to enable downlinking. The analysis that calculates the communication time daily, shows that the CubeSat will be in communication with the ground station for 11 minutes per 24 hour cycle. Based on a data rate on $9.6 \mathrm{kbps}$, there will more raw data than is capable of being downlinked. This would require the CubeSat to downsample the data, and likely also include some compression capability in order to transmit all generated data in a 24 hour period.

The dynamics model shows that the initial concept of intentionally inducing a tumble with the test thrusters acts as intended. Short firings result in a measurable dynamic 
event which can be read accurately by reasonably priced hardware. The analysis includes all realistic variables in order to ensure that any scenario experienced in the flight tests is traceable to this model, thus allowing the determination of the realistic thruster performance. A key aspect through this analysis is the need to accurately discern the inertial tensor. The tensor has a large effect on the end dynamic result and using a tensor with a large error could lead to the miscalculation of the thrust performance thus skewing the intention of the mission. The analysis also highlights the effect of having an unbalanced inertia tensor, in that the craft experiences more drastic complex tumbles for a given torque as the craft becomes more unbalanced. In the worst case scenario where the craft is assembled with little concern to balance, the scenario in which the thruster is perfectly placed still results in a tumble about an unintended axis almost equal to the intended axis. This would also realistically cause significant difficulty in re-balancing the craft after each firing. This poses a risk of mission failure as the craft entering an unrecoverable tumble would result in intermittent power generation and result in the depletion of the batteries preventing the execution of the mission. 


\section{BIBLIOGRAPHY}

[1] The CubeSat Program. CubeSat Design Specification, volume 13. California Polytechnic State University.

[2] J. Puig-Suari, C. Turner, and W. Ahlgren. Development of the standard cubesat deployer and a cubesat class picosatellite. 2001 IEEE Aerospace Conference Proceedings, Mar 2001.

[3] Michael Swartwout. The first one hundred cubesats: A statistical look. Journal of small satellites, 2(2), 2013.

[4] Halis Polat, Josep Virgili-Llop, and Marcello Romano. Survey, statistical analysis and classification of launched cubesat missions with emphasis on the attitude control method. Journal of small satellites, 5(3).

[5] NASA Technical Standards. Process for Limiting Orbital Debris, NASA-STD 8719.14. NASA.

[6] Juergen Mueller, Richard Hofer, and John Ziemer. Survey of propulsion technologies applicable to cubesats. Joint Army-Navy-NASA-Air Force (JANNAF), May 2010.

[7] Kristina Lemmer. Propulsion for cubesats. Acta Astronautica, (134), 2017.

[8] John Ziemer, Colleen Marrese-Reading, and Charley Dunn. Colloid microthruster flight performance results from space technology 7 disturbance reduction system. The 35th International Electric Propulsion Conference, Oct 2017. 
[9] Jian Guo, Jasper Bouwmeester, and Eberhard Gill. In-orbit results of delfi-n3xt: Lessons learned and move forward. Acta Astronautica, (121).

[10] David Krejci, Alexander Reissner, Bernhard Seifert, David Jelem, Thomas Horbe, Florin Plesescu, Pete Friedhoff, and Steve Lai. Demonstration of the ifm nano feep thruster in low earth orbit. The 4 S Symposium, 2018.

[11] Joyce Wong. The university micro/nanosatellite as a micropropulsion testbed. 13" Annual AIAAfUSU Conference on Small Satellites.

[12] C Charles. Plasmas for spacecraft propulsion. Journal of Physics D: Applied Physics, 42(16):163001, 2009.

[13] C Charles and R W Boswell. Measurement and modelling of a radiofrequency micro-thruster. Plasma Sources Science and Technology, 21(2):022002, 2012.

[14] Christine Charles, Teck-Seng Ho, Ashley Ellis, Thomas Charoy, Alex Bennet, Alex Stutchbery, Rod Boswell, Wei Liang, Luke Raymond, Juan Rivas-Davila, and et al. The pocket rocket electro-thermal plasma thruster for 'cubesat' nano-satellites. International Astronautical Congress, Sep 2017.

[15] Wei Liang, Christine Charles, Luke Raymond, Alex Stuchbery, Kawin Surakitbovorn, Lei Gu, Rod Boswell, and Juan Rivas-Davila. An integrated rf power delivery and plasma micro-thruster system for nano-satellites. Frontiers in Physics, Oct 2018.

[16] Sebastian Gnagy, Alec Henken, and Amelia Greig. Cubesat electrothermal plasma micro-thruster: System development and integration. 32nd Annual AIAA/USU Conference on Small Satellites, Aug 2018. 
[17] Alec Henken. Thermal modeling and validation of heat profiles in an rf plasma micro-thruster. 2018.

[18] Liam Bruno. Polysat info. Personal Communication, Nov 2018.

[19] Amelia Diane Greig et al. Pocket rocket: An electrothermal plasma micro-thruster. 2015.

[20] Active satellites. CelesTrak.

[21] David G. Gilmore and Martin Donabedian. Spacecraft thermal control handbook. American Institute of Aeronautics and Astronautics, 2003.

[22] Justin Nguyen. Questions about polysat ground station. Personal Communication, Apr 2019.

[23] Wiley J Larson and James Richard Wertz. Space mission analysis and design. Technical report, Torrance, CA (United States); Microcosm, Inc., 1992.

[24] Austin Williams. A compact, reconfigurable uhf communication system design for use with polysats embedded linux platform. 2013. 